\title{
Birational geometry of compactifications of Drinfeld half-spaces over a finite field
}

\author{
Adrian Langer
}

February 18, 2019

\author{
ADDRESS: \\ Institute of Mathematics, University of Warsaw, ul. Banacha 2, 02-097 Warszawa, Poland \\ e-mail: alan@mimuw.edu.pl
}

\begin{abstract}
We study compactifications of Drinfeld half-spaces over a finite field. In particular, we construct a purely inseparable endomorphism of Drinfeld's half-space $\Omega(V)$ over a finite field $k$ that does not extend to an endomorphism of the projective space $\mathbb{P}(V)$. This should be compared with theorem of Rémy, Thuillier and Werner that every $k$-automorphism of $\Omega(V)$ extends to a $k$-automorphism of $\mathbb{P}(V)$. Our construction uses an inseparable analogue of the Cremona transformation. We also study foliations on Drinfeld's half-spaces. This leads to various examples of interesting varieties in positive characteristic. In particular, we show a new example of a non-liftable projective Calabi-Yau threefold in characteristic 2 and we show examples of rational surfaces with klt singularities, whose cotangent bundle contains an ample line bundle.
\end{abstract}

2010 Mathematics Subject Classification. Primary 14G17; Secondary 14E10, 14J26

\section{Introduction}

Let $k$ be a finite field and let $\Omega\left(k^{n+1}\right)$ be Drinfeld's half-space, i.e., the complement of all $k$-rational hyperplanes in $\mathbb{P}^{n}$. The main aim of the paper is to study various compactifications of $\Omega(V)$ and their purely inseparable quotients from the point of view of birational geometry. In this way we obtain a few interesting examples of varieties or maps defined over finite fields.

Our first result describes endomorphisms of Drinfeld's half-space.

THEOREM 1.1. 1. Every separable dominant k-endomorphism $\psi$ of $\Omega\left(k^{n+1}\right)$ is a k-automorphism and it extends to a $k$-automorphism of $\mathbb{P}^{n}$.

2. There exists a radicial $k$-endomorphism $\psi$ of $\Omega\left(k^{n+1}\right)$ that does not extend to a $k$-endomorphism of $\mathbb{P}^{n}$.

This should be compared with the main result of [28] that says that every $k$-automorphism of $\Omega\left(k^{n+1}\right)$ extends to a $k$-automorphism of $\mathbb{P}^{n}$. The proof of this fact is done in two steps: first the authors use Berkovich spaces to prove that every $k$-automorphism of $\Omega\left(k^{n+1}\right)$ extends to a $k$-automorphism of the wonderful compactification $\tilde{X}$ of $\Omega\left(k^{n+1}\right)$ and then they use intersection theory to prove that one can descend this automorphism to the projective space. We give a new proof of the first step without Berkovich spaces following an idea that the author learnt from H. Esnault and V. Srinivas. Namely, the result follows if one proves that $K_{\tilde{X}}+D$ is ample for the complement $D$ of $\Omega\left(k^{n+1}\right)$ in $\tilde{X}$. This fact goes back to Iitaka (at least in the characteristic zero case; see [17, Theorem 11.6]). Ampleness of $K_{\tilde{X}}+D$ is claimed by Mustafin

Author's work was partially supported by Polish National Science Centre (NCN) contract numbers 2013/08/A/ST1/00804 and 2015/17/B/ST1/02634. 
in [25, p. 227, Lemma], but his proof shows only that it is strictly nef (he checks only that the degree of $K_{\tilde{X}}+D$ on curves is positive). We prove ampleness of this line bundle by showing that $K_{\tilde{X}}+D=(|k|-1) L$ for some line bundle $L$ such that the complete linear system $|L|$ is the composition of embedding of $\tilde{X}$ into the full flag variety (as a Deligne-Lusztig variety), then embedding the full flag variety into a product of projective spaces (via Plücker embeddings of partial flags) and finally embedding this product by the Segre embedding into some projective space.

To show the second part of the theorem we construct a new natural compactification $X$ of $\Omega\left(k^{n+1}\right)$ (which is non-normal if $n \geq 3$ ) to which one can extend $\psi$, so that we have a commutative diagram

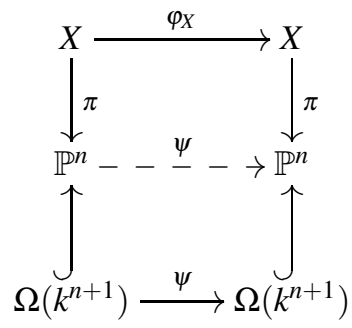

in which $\varphi_{X}$ is a purely inseparable morphism such that $\varphi_{X}^{2}$ is the composition of $(n-1) k$-linear Frobenius endomorphisms $\operatorname{Fr}_{X}$ of $X$. This can be considered as a kind of an "inseparable flop" exchanging the role played by $k$-rational codimension $i$ subspaces of $\mathbb{P}^{n}$ and $k$-rational codimension $(n+1-i)$ subspaces of $\mathbb{P}^{n}$. We also have a similar diagram with $X$ replaced by the wonderful compactification $\tilde{X}$ of $\Omega\left(k^{n+1}\right)$ but then the corresponding endomorphism $\varphi_{\tilde{X}}$ is not so easy to see directly.

Taking Stein's factorization of $\pi \circ \varphi_{X}$ we get a birational morphism $f: X \rightarrow Y$ and a purely inseparable morphism $\varphi_{Y}: Y \rightarrow \mathbb{P}^{n}$. The fact that $\varphi_{X}^{2}=\operatorname{Fr}_{X}^{\operatorname{dim} V-2}$ leads to a new purely inseparable morphism $\varphi: \mathbb{P}^{n} \rightarrow$ $Y$. In particular, we have the following more precise version of the second part of Theorem 1.1

THEOREM 1.2. We have the following commutative diagram of $k$-schemes

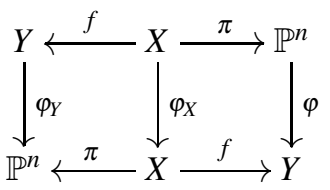

in which horizontal maps are birational, vertical maps are purely inseparable and they satisfy the following relations

$$
\varphi_{X}^{2}=\operatorname{Fr}_{X}^{n-1}, \quad \varphi_{Y} \circ \varphi=\operatorname{Fr}_{\mathbb{P}^{n}}^{n-1}, \quad \varphi \circ \varphi_{Y}=\operatorname{Fr}_{Y}^{n-1} .
$$

The map $\psi=\pi \circ \varphi_{X} \circ \pi^{-1}: \mathbb{P}^{n} \rightarrow \mathbb{P}^{n}$ is purely inseparable and it satisfies $\psi^{2}=\mathrm{Fr}_{\mathbb{P}^{n}}^{n-1}$. Moreover, $\psi$ defines an endomorphism of $\Omega\left(k^{n+1}\right)$.

In the above theorem Fr stands for the $k$-linear Frobenius endomorphism ("raising to $q$-th power" for $k=\mathbb{F}_{q}$ ). In fact, our construction of schemes $X, Y$ and maps $\psi, \varphi_{X}$ etc. is functorial in the $k$-vector space $V=k^{n+1}$ but the precise statements become more complicated (see Theorem 5.6) and we get a morphism $\psi_{V}: \Omega(V) \rightarrow \Omega\left(V^{*}\right)$ rather than an endomorphism of $\Omega(V)$.

We can look at $\psi$ as an analogue of the Cremona transformation, which can be recovered by specializing to "characteristic 1". We illustrate this in dimension $n=2$. Let us recall that the Cremona transformation is defined as the blow up of 3 distinct points of $\mathbb{P}^{2}$ and then blowing down the strict transforms of 3 lines between any two of the blown up points. Similarly, $\psi$ is obtained by first blowing up all $k$-rational points of $\mathbb{P}^{2}$, performing an inseparable endomorphism $\varphi_{X}$ and then contracting images of the strict transforms of all $k$-rational lines. A slightly different way to see the analogy is the following. The Cremona transformation can be defined by choosing 3 distinct points of $\mathbb{P}^{2}$ and taking the map defined by products of equations of lines passing through 2 of these points. To define $\psi$ in coordinates we need to choose 3 distinct $k$-rational points of $\mathbb{P}^{2}$ and take the products of equations of all $k$-rational lines passing through these points. For 
example, for a standard choice of points $[1,0,0],[0,1,0],[0,0,1]$ in $\mathbb{P}^{2}$, the Cremona transformation is given by

$$
\left[x_{0}, x_{1}, x_{2}\right] \rightarrow\left[x_{1} x_{2}, x_{2} x_{0}, x_{0} x_{1}\right]
$$

whereas $\psi$ is given by

$$
\left[x_{0}, x_{1}, x_{2}\right] \rightarrow\left[w_{0}, w_{1}, w_{2}\right]=\left[x_{1} x_{2}^{q}-x_{1}^{q} x_{2}, x_{2} x_{0}^{q}-x_{0} x_{2}^{q}, x_{0} x_{1}^{q}-x_{1} x_{0}^{q}\right] .
$$

This definition makes it rather non-obvious that $\psi^{2}$ is given by $\left[x_{0}, x_{1}, x_{2}\right] \rightarrow\left[x_{0}^{q}, x_{1}^{q}, x_{2}^{q}\right]$ or that $\psi \circ \pi$ factors through a purely inseparable morphism $X \rightarrow X$.

We construct $X$ as a complete intersection in the product of two projective spaces. Then we can easily construct $\varphi_{X}$ as restriction of a certain morphism on the product. Finally, we check that it preserves the boundary divisor producing an endomorphism of $\Omega\left(k^{n+1}\right)$.

A large part of the paper is devoted to the study of 1-foliations related to Drinfeld's compactification. More precisely, the logarithmic tangent sheaf of $\mathbb{P}^{n}$ with poles along complement of $\Omega\left(k^{n+1}\right)$ splits into a direct sum of line bundles. The direct sums of these line bundles define 1-foliations of arbitrary rank on $\mathbb{P}^{n}$ and singularities of these 1 -foliations can be resolved by successive blowing ups along $\mathbb{F}_{q}$-rational linear spaces of fixed codimension. In case $k=\mathbb{F}_{p}$ and rank 1 we obtain the 1 -foliation considered in [13] to construct a non-liftable Calabi-Yau threefold in characteristic 3. For $k=\mathbb{F}_{2}$ we can use our rank 2 1-foliation to construct a new example of a non-liftable projective Calabi-Yau threefold.

THEOREM 1.3. There exists a smooth, projective Calabi-Yau 3-fold $\tilde{Y} / \overline{\mathbb{F}}_{2}$ such that the following conditions are satisfied:

1. $b_{2}(\tilde{Y})=51$ and $h^{1}\left(\mathscr{O}_{\tilde{Y}}\right)=h^{2}\left(\mathscr{O}_{\tilde{Y}}\right)=0$,

2. $\tilde{Y}$ is unirational,

3. $h^{0}\left(T_{\tilde{Y}}\right)=0$,

4. $T_{\tilde{Y}}$ is not semistable with respect to some ample polarizations,

5. $\tilde{Y}$ does not admit a formal lifting to characteristic zero.

In the above theorem $b_{i}(X)$ denotes the $i$-th Betti number of $X$, i.e., the dimension of $i$-th $l$-adic cohomology group of $X$ for some $l \neq p$.

Note that till now the only smooth projective Calabi-Yau 3-folds that are not liftable to characteristic zero were constructed in characteristic 2 and 3 (see, e.g., [13], [30], [4]). There exist also non-projective examples in other characteristics (see [4]). It seems that constructions in characteristic 2 are somewhat more complicated than those in characteristic 3 .

Before stating other applications of our result, let us recall that in any characteristic the cotangent bundle of a separably uniruled smooth proper variety does not contain any big line bundles (see [19, Chapter V, Lemma 5.1]). This was used by J. Kollár (see [19, Chapter V, Theorem 5.14]) and in various subsequent papers to prove non-rationality of some complex Fano varieties. Moreover, it is known that if $Y$ is a complex projective variety with only klt singularities then $\hat{\Omega}_{Y}=\left(\Omega_{Y}\right)^{* *}$ does not contain any big $\mathbb{Q}$-Cartier Weil divisors (see [10, Theorem 7.2]). Here we show that in positive characteristic $\hat{\Omega}_{Y}$ can contain ample line bundles, even if $Y$ has only klt singularities and it is separably uniruled. More precisely, we have the following theorem:

THEOREM 1.4. Let $k=\mathbb{F}_{q}$ be a finite field of characteristic p. There exists a geometrically normal projective surface $Y_{0} / k$ such that $Y=\left(Y_{0}\right)_{\bar{k}}$ has the following properties:

1. $Y$ is rational,

2. Y has only klt singulartities of type a cone over $\left(\mathbb{P}^{1}, \mathscr{O}_{\mathbb{P}^{1}}(q)\right)$,

3. $\rho(Y)=1$, 
4. $\hat{\Omega}_{Y}$ contains an ample line bundle,

5. $T_{Y}$ is not generically semi-negative,

6. if $p=2$ then $-K_{Y}$ is ample; moreover, if $q=2$ then $T_{Y}$ is locally free,

7. if $p>2$ then $K_{Y}$ is ample.

This theorem is related to the following proposition:

Proposition 1.5. Let $g: \tilde{S}=\mathbb{V}\left(\mathscr{O}_{\mathbb{P}^{1}}(-d)\right) \rightarrow S=\operatorname{Spec} \bigoplus_{i \geq 0} H^{0}\left(\mathbb{P}^{1}, \mathscr{O}(i d)\right)$ be the resolution of a cone over $\left(\mathbb{P}^{1}, \mathscr{O}_{\mathbb{P}^{1}}(d)\right)$ in characteristic p dividing $d$. Then $S$ has only klt, $F$-regular singularities but $g_{*} \Omega_{\tilde{S}}$ is not reflexive.

This should be contrasted with the main result of [10, Theorem 1.4], which says that over complex numbers if $g: \tilde{Z} \rightarrow Z$ is a $\log$ resolution a quasi-projective variety $Z$ with at most klt singularities then $g_{*} \Omega_{\tilde{Z}}$ is reflexive.

\section{Relation to other work.}

Let $V$ be an $(n+1)$-dimensional vector space over a finite field $k$. After most of the paper was written, the author noticed a related paper [27] of R. Pink and S. Schieder in which the authors construct some purely inseparable maps $g_{V}: \Omega(V) \rightarrow \Omega\left(V^{*}\right)$ and $f_{V}: \Omega\left(V^{*}\right) \rightarrow \Omega(V)$ (see [27, Proposition 9.1]; note that most of the proof is skipped and "lengthy elementary calculations" are left to the reader).

Note that in their case $f_{V} g_{V}=\operatorname{Fr}_{\Omega(V)}^{n}$ and $g_{V} f_{V}=\operatorname{Fr}_{\Omega\left(V^{*}\right)}^{n}$, whereas we construct a map $\psi_{V}: \Omega(V) \rightarrow$ $\Omega\left(V^{*}\right)$ such that $\psi_{V^{*}} \circ \psi_{V}=\operatorname{Fr}_{\Omega(V)}^{n-1}$ (see Theorem 5.6, which is a more precise version of Theorem 1.2). It is rather easy to see that $f_{V}=\psi_{V}$ and after asking about relation between the results, the author was informed by R. Pink that after some computations one can show that $g_{V^{*}}=f_{V} \circ \mathrm{Fr}_{: \Omega\left(V^{*}\right)}$. In particular, [27] Proposition 9.1] agrees with Theorem 5.6 Our approach and description of the map $\psi_{V}$ is different and more geometric than that of $f_{V}$, showing its relation to the Cremona transformation.

S. Kurul and A. Werner proved in a recent preprint [18] that all endomorphisms of Drinfeld's halfspace extend to the wonderful compactification. In fact, their method allows to deal also with more general complements of hyperplane arrangements in $\mathbb{P}^{n}$.

Theorem 1.4 comes from a detailed study of the example in [20, Section 8] and from the related paper [3] by Cascini and Tanaka. More precisely, in [20] the author showed that if $D$ on $X$ considered above is the sum of strict transforms of $k$-rational lines then $\Omega_{X}(\log D)$ contains a big line bundle with positive self-intersection. However, this was only an existence result proven using non-trivial methods. Here we produce such a line bundle bundle explicitly using some computations involving certain foliation on $X$. Then we push it down to surface $Y$ obtained by contracting $D$ and studied in [3] (see [3, Lemma 2.4]).

\section{Notation}

Let $k$ be a finite field with $q=p^{e}$ elements and let $\operatorname{Fr}_{k}: \bar{k} \rightarrow \bar{k}$ be the Frobenius automorphism of $\bar{k}$ defined by $\operatorname{Fr}_{k}(a)=a^{q}$. Clearly, $\operatorname{Fr}_{k}$ is identity on $k \subset \bar{k}$.

Let $X$ be a scheme of characteristic $p$. The absolute Frobenius morphism $F_{X}: X \rightarrow X$ is defined as identity on topological spaces and raising to $p$-th power on structure sheaves. For a $k$-scheme $X$ we define a $k$-scheme $X^{(i)}$ as the base change of $X / k$ via $k \rightarrow k$ defined by $a \rightarrow a^{p^{i}}$. Then we get a $k$-linear morphism $F^{i}: X \rightarrow X^{(i)}$ induced by $\left(F_{X}\right)^{i}: X \rightarrow X$, i.e., composition of $i$ absolute Frobenius morphisms $F_{X}$. Since $\operatorname{Fr}_{k}=\left(F_{k}\right)^{e}$ is identity on $k$, the $k$-schemes $X$ and $X^{(e)}$ can be identified and we have a $k$-linear endomorphism $\mathrm{Fr}_{X}=F^{e}: X \rightarrow X^{(e)}=X$.

If $V$ is a finite dimension $k$-vector space then we set $\mathbb{P}(V)=\operatorname{Proj}\left(\operatorname{Sym}^{\bullet} V\right)$. More generally, if $E$ is a locally free sheaf on a scheme $X$ then we set $\mathbb{P}(E)=\operatorname{Proj}\left(\operatorname{Sym}^{\bullet} E\right)$.

A scheme structure on a $k$-vector space $W$ is given by $W=\operatorname{Spec} \operatorname{Sym}^{\bullet} W^{*}$. By definition we have the standard projection map $V^{*}-\{0\} \rightarrow \mathbb{P}(V)$. This is a quotient by the canonical $\mathbb{G}_{m}$-action on $V^{*}$ (which is 
free on $V^{*}-\{0\}$ ). Linear coordinates in $V^{*}$ (i.e., affine coordinates vanishing at 0 or a choice of basis of $V)$ give homogeneous coordinates in $\mathbb{P}(V)$. If we choose homogeneous coordinates $x_{0}, \ldots, x_{n}$ in $\mathbb{P}(V)$ and take an element $f \in k\left[x_{0}, \ldots, x_{n}\right]$ then we denote by $D_{+}(f)$ the principal open subset corresponding to $f$, i.e., $D_{+}(f)=\left\{\mathfrak{p} \in \mathbb{P}(V)=\operatorname{Proj} k\left[x_{0}, \ldots, x_{n}\right] \mid f \notin \mathfrak{p}\right\}$.

\section{Preliminaries}

\subsection{Moore determinant}

The Moore determinant $\Delta_{q}\left(w_{1}, \ldots, w_{n}\right)$ is defined as the determinant of the matrix

$$
\left(\begin{array}{ccc}
w_{1} & \ldots & w_{n} \\
w_{1}^{q} & \ldots & w_{n}^{q} \\
\vdots & & \vdots \\
w_{1}^{q^{n-1}} & \ldots & w_{n}^{q^{n-1}}
\end{array}\right)
$$

treated as an element of $\mathbb{F}_{q}\left[w_{1}, \ldots, w_{n}\right]$.

LEMMA 2.1. (see [9. Corollary 1.3.7]) Moore determinant is equal to the product of all linear forms over $\mathbb{F}_{q}$ with the last non-zero coefficient equal to 1 , i.e., we have

$$
\Delta_{q}\left(w_{1}, \ldots, w_{n}\right)=\prod_{i=1}^{n} \prod_{a_{1} \in \mathbb{F}_{q}} \ldots \prod_{a_{i-1} \in \mathbb{F}_{q}}\left(a_{1} w_{1}+\ldots+a_{i-1} w_{i-1}+w_{i}\right)
$$

in $\mathbb{F}_{q}\left[w_{1}, \ldots, w_{n}\right]$.

COROLlary 2.2. We have

$$
\frac{\partial \Delta_{q}}{\partial w_{i}}\left(w_{1}, \ldots, w_{n}\right)=(-1)^{i+1}\left(\Delta_{q}\left(w_{1}, \ldots, \hat{w}_{i}, \ldots, w_{n}\right)\right)^{q} .
$$

Proof. Using expansion of the defining matrix with respect to the $i$-th column, we get

$$
\frac{\partial \Delta_{q}}{\partial w_{i}}\left(w_{1}, \ldots, w_{n}\right)=(-1)^{i+1} \Delta_{q}\left(w_{1}^{q}, \ldots, \hat{w_{i}^{q}}, \ldots, w_{n}^{q}\right)
$$

Since

$$
a_{1} w_{1}^{q}+\ldots+a_{i-1} w_{i-1}^{q}+w_{i}^{q}=\left(a_{1} w_{1}+\ldots+a_{i-1} w_{i-1}+w_{i}\right)^{q}
$$

for $a_{1}, \ldots, a_{i-1} \in \mathbb{F}_{q}$, the required equality follows from Lemma 2.1 .

\subsection{Graphs of rational maps and blow-ups}

Let $X$ be an integral scheme defined over some field $k$ and let $\mathscr{L}$ be an invertible sheaf on $X$. Let us consider a finite dimensional $k$-subspace $W \subset H^{0}(X, \mathscr{L})$ and let

$$
u: W \otimes_{k} \mathscr{O}_{X} \subset H^{0}(X, \mathscr{L}) \otimes_{k} \mathscr{O}_{X} \rightarrow \mathscr{L}
$$

be the restriction of the evaluation map. The image of the induced map $W \otimes_{k} \mathscr{L}^{-1} \rightarrow \mathscr{O}_{X}$ is an ideal sheaf of some subscheme $Y \subset X$. The following lemma is certainly well known but we prove it for lack of an appropriate reference.

LEMMA 2.3. The blow up of $X$ along $Y$ is the schematic closure in $X \times{ }_{k} \mathbb{P}(W)$ of the graph of the map $U=X-Y \rightarrow \mathbb{P}(W)$ defined by the linear system corresponding to $W$. 
Proof. Let $\mathscr{A}=\bigoplus_{d \geq 0} I_{Y}^{d}$ and let $\mathscr{A} * \mathscr{L}$ be a sheaf of graded $\mathscr{O}_{X}$-algebras defined by $(\mathscr{A} * \mathscr{L})_{d}=\mathscr{A}_{d} \otimes_{\mathscr{O}_{X}}$ $\mathscr{L}^{\otimes d}$ for $d \geq 0$. The surjective map $W \otimes_{k} \mathscr{O}_{X} \rightarrow I_{Y} \otimes_{\mathscr{O}_{X}} \mathscr{L}$ defines a surjective map of sheaves of graded $\mathscr{O}_{X}$-algebras

$$
\operatorname{Sym}^{\bullet}\left(W \otimes_{k} \mathscr{O}_{X}\right) \rightarrow \mathscr{A} * \mathscr{L},
$$

that preserves degrees. It gives rise to a closed immersion

$$
Z:=\operatorname{Proj} \mathscr{A} \simeq \operatorname{Proj} \mathscr{A} * \mathscr{L} \hookrightarrow \operatorname{Proj} \operatorname{Sym}^{\bullet}\left(W \otimes_{K} \mathscr{O}_{X}\right)=\mathbb{P}(W)_{X}
$$

of the blow up $Z$ of $X$ along $Y$ into $\mathbb{P}(W)_{X}$. Since $X$ is integral, $Z$ is also integral. Therefore $Z$ is the schemetheoretic closure in $X \times_{X} \mathbb{P}(W)_{X}=\mathbb{P}(W)_{X}$ of the graph of the morphism $U=X-Y \rightarrow \mathbb{P}(W)_{X}$ defined by surjection $\left.W \otimes_{k} \mathscr{O}_{U} \rightarrow \mathscr{L}\right|_{U}$. Since $\mathbb{P}(W)_{X}=X \times_{k} \mathbb{P}(W)$, this is equivalent to taking the scheme-theoretic closure of the graph of the morphism $U \rightarrow \mathbb{P}(W)$ defined by the linear system corresponding to $W$.

Lemma 2.4. Let $X$ be a scheme and let $Y_{1}, Y_{2} \subset X$ be subschemes. Let $f_{i}: Z_{i} \rightarrow X$ be the blow-ups of $X$ along $Y_{i}$ for $i=1,2$ and let $W_{i}$ be the blow up of $Z_{i}$ along $f_{i}^{-1}\left(Y_{j}\right)$, where $\{i, j\}=\{1,2\}$. Then $W_{1}$ is isomorphic to $W_{2}$ as an $X$-scheme and both of them dominate the blow up of $X$ along the subscheme $Y_{1} \cup Y_{2} \subset X$.

Proof. The first part of the lemma is standard (see, e.g., [23, Lemma 3.2]). To prove the second one note that both $\left(g_{1} \circ f_{1}\right)^{-1}\left(Y_{1}\right) \subset W_{1}$ and $\left(g_{1} \circ f_{1}\right)^{-1}\left(Y_{2}\right) \subset W_{1}$ are Cartier divisors. Since the ideal sheaf of $Y_{1} \cup Y_{2}$ is the intersection of ideal sheaves of $Y_{1}$ and $Y_{2}$ in $\mathscr{O}_{X}$, also $\left(g_{1} \circ f_{1}\right)^{-1}\left(Y_{1} \cup Y_{2}\right) \subset W_{1}$ is a Cartier divisor. Hence the required assertion follows from the universal property of the blow-up.

\subsection{Wonderful compactifications}

Let $X$ be a smooth algebraic variety defined over some perfect field $k$. An arrangement of subvarieties $\mathscr{L}$ is a finite collection of smooth subvarieties such that all nonempty scheme-theoretic intersections of subvarieties in $\mathscr{L}$ are in $\mathscr{L}$. Any such arrangement is a poset with order defined by inclusion. Let us set $X^{o}:=X-\bigcup_{Y \in \mathscr{L}} Y$. A subset $\mathscr{B} \subset \mathscr{L}$ is called a building set if for all $Y \in \mathscr{L}-\mathscr{B}$ the minimal elements in $\{Z \in \mathscr{B}: Y \subset Z\}$ intersect transversally and their intersection is equal to $Y$.

For any arrangement $\mathscr{L}$ one can define the wonderful compactification of $X^{o}$ as the scheme-theoretic closure of the image of the natural locally closed embedding

$$
X^{o} \hookrightarrow \prod_{Y \in \mathscr{L}} \mathrm{Bl}_{Y} X
$$

The following theorem is a summary of various results starting with [5], [15] and finishing with [23, Theorem 1.2 and Theorem 1.3]. We state it only in a special case when a building set is equal to the arrangement. This is the only case that is interesting from the point of view of compactification of Drinfeld's half-space.

THEOREM 2.5. Wonderful compactification $\tilde{X}$ of $X^{o}$ is a smooth algebraic k-variety isomorphic to the blow up of $X$ along the product of ideal sheaves of $Y \in \mathscr{L}$. For each $Y \in \mathscr{L}$ there is a smooth divisor $D_{Y} \subset \tilde{X}$ such that $\tilde{X}-X^{0}=\bigcup_{Y \in \mathscr{L}} D_{Y}$ is a simple normal crossings divisor and an intersection of divisors $D_{Y_{1}} \cap D_{Y_{2}} \cap \ldots \cap D_{Y_{m}}$ is nonempty if and only if $D_{Y_{1}}, D_{Y_{2}}, \ldots, D_{Y_{m}}$ form a chain in the poset $\mathscr{L}$. Moreover, if we order elements of $\mathscr{L}$ in such a way that for any $i$ the first $i$ elements of the order $D_{1}, \ldots, D_{N}$ form a building set then

$$
\tilde{X}=\mathrm{Bl}_{\tilde{D}_{N}} \cdots \mathrm{Bl}_{\tilde{D}_{2}} \mathrm{Bl}_{D_{1}} X,
$$

where $\tilde{D}_{i}$ are defined inductively as $f_{i}^{-1}\left(D_{i}\right)$ for $f_{i}: \mathrm{Bl}_{\tilde{D}_{i-1}} \ldots \mathrm{Bl}_{D_{1}} X \rightarrow X$.

\subsection{Foliations}

Let $X$ be a normal variety defined over some field $k$. A foliation on $X$ is a saturated $\mathscr{O}_{X}$-submodule $\mathscr{F} \subset$ $T_{X / k}=\operatorname{Der}_{k}\left(\mathscr{O}_{X}, \mathscr{O}_{X}\right)$ preserved by the Lie bracket. If $\mathscr{F} \subset T_{X / k}$ is a foliation then by definition $T_{X / k} / \mathscr{F}$ is 
torsion free and hence $\mathscr{F}$ is reflexive. In particular, if $X$ is a surface then $\mathscr{F}$ is locally free. If $X$ is smooth and $T_{X / k} / \mathscr{F}$ is locally free then we say that $\mathscr{F}$ is a smooth foliation.

Let $f: X_{2} \rightarrow X_{1}$ be a birational morphism between normal $k$-varieties. Then a foliation $\mathscr{F}_{1} \subset T_{X_{1} / k}$ induces on $X_{2}$ a foliation $\mathscr{F}_{2} \subset T_{X_{2} / k}$ (of the same rank as $\mathscr{F}_{1}$ ) by taking the kernel of

$$
T_{X_{2} / k} \rightarrow f^{*} T_{X_{1} / k} \rightarrow f^{*}\left(T_{X_{1} / k} / \mathscr{F}_{1}\right) / \text { Torsion }
$$

By assumption the first map is injective, so if $\mathscr{F}_{1}$ is locally free then $\mathscr{F}_{2}$ is the saturation of $f^{*} \mathscr{F}_{1} \cap T_{X_{2} / k}$ in $T_{X_{2} / k}$. Note that condition $\left[\mathscr{F}_{1}, \mathscr{F}_{1}\right] \subset \mathscr{F}_{1}$ implies that $\left[\mathscr{F}_{2}, \mathscr{F}_{2}\right] \subset \mathscr{F}_{2}$. Indeed, the map $\bigwedge^{2} \mathscr{F}_{2} \rightarrow T_{X_{2} / k} / \mathscr{F}_{2}$ is $\mathscr{O}_{X_{2}}$-linear and zero at the generic point of $X_{2}$, so it is the zero map.

The above construction shows that we can try to resolve singularities of a foliation $\mathscr{F}_{1} \subset T_{X_{1} / k}$ and try to construct a proper birational map $f: X_{2} \rightarrow X_{1}$ from a smooth variety $X_{2}$ such that the induced foliation is smooth. In general, this is not possible even for foliations on smooth complex surfaces (see [31]). However, all the foliations considered in this paper have nice resolutions by smooth foliations.

\subsection{1-foliations in positive characteristic}

Let $X$ be a normal variety defined over some field $k$ of positive characteristic $p$. If $x$ is a $k$-derivation of the sheaf of rings $\mathscr{O}_{X}$ then the differential operator $x^{p}$ acts on $\mathscr{O}_{X}$ as a derivation. The corresponding derivation is denoted by $x^{[p]}$. Thus we have a well defined $p$-th power map ${ }^{[p]}: T_{X / k} \rightarrow T_{X / k}$.

We say that a foliation $\mathscr{F} \subset T_{X / k}$ is a 1 -foliation, if $\mathscr{F}[p] \subset \mathscr{F}$.

If $f: X_{2} \rightarrow X_{1}$ is a birational morphism of smooth $k$-varieties then a 1 -foliation $\mathscr{F}_{1} \subset T_{X_{1} / k}$ induces a 1-foliation $\mathscr{F}_{2} \subset T_{X_{2} / k}$. To check this one needs to remark that the map $F_{X_{2}}^{*} \mathscr{F}_{2} \rightarrow T_{X_{2} / k} / \mathscr{F}_{1}$ is $\mathscr{O}_{X_{2}}$-linear and since $\mathscr{F}_{1}^{[p]} \subset \mathscr{F}_{1}$, this map is zero at the generic point of $X_{2}$. Therefore it is the zero map and hence $\mathscr{F}_{2}^{[p]} \subset \mathscr{F}_{2}$.

In positive characteristic, there always exist quotients by 1 -foliations. More precisely, let $\mathscr{F} \subset T_{X / k}$ be a 1-foliation. Then we can define a sheaf $\mathscr{O}_{Y}=\mathscr{O}_{X}^{\mathscr{F}}$ of abelian groups by setting

$$
\mathscr{O}_{Y}(U)=\left\{x \in \mathscr{O}_{X}(U): \forall V \subset U \text { open, } \forall s \in \mathscr{F}(V) \quad s\left(\left.x\right|_{V}\right)=0\right\}
$$

for an open subset $U \subset X$. One can check that $\mathscr{O}_{Y}$ is a sheaf of $k$-algebras so it defines a scheme $Y$, which has $X$ as the underlying topological space and $\mathscr{O}_{Y}$ as a structure sheaf. The inclusion $\mathscr{O}_{Y} \subset \mathscr{O}_{X}$ defines the map $f: X \rightarrow Y$ called a quotient by 1 -foliation $\mathscr{F}$. Usually, we denote $Y$ by $X / \mathscr{F}$. Since $\mathscr{O}_{X}^{p} \subset \mathscr{O}_{Y}$, the $p$ th power map $F_{X}^{\#}: \mathscr{O}_{X} \rightarrow \mathscr{O}_{X}$ factors through $\mathscr{O}_{Y} \subset \mathscr{O}_{X}$, so there exists a map $g: Y \rightarrow X$ such that $g f=F_{X}$. Clearly, also $F_{Y}^{\#}: \mathscr{O}_{Y} \rightarrow \mathscr{O}_{Y}$ factors through $\mathscr{O}_{Y} \subset \mathscr{O}_{X}$, so $f g=F_{Y}$.

Let us define the map of sheaves of abelian groups

$$
\varphi: \mathscr{O}_{X} \rightarrow \mathscr{F}^{*}=\mathscr{H} \operatorname{om}_{\mathscr{O}_{X}}\left(\mathscr{F}, \mathscr{O}_{X}\right)
$$

by $(\varphi(x))(s)=s(x)$ for $x \in \mathscr{O}_{X}$ and $s \in \mathscr{F} \subset \operatorname{Der}_{k}\left(\mathscr{O}_{X}, \mathscr{O}_{X}\right)$. The map $\varphi$ can be also described as a composition of the universal derivation $d: \mathscr{O}_{X} \rightarrow \Omega_{X / k}$ with an $\mathscr{O}_{X}$-linear map $\Omega_{X / k} \rightarrow \mathscr{F}^{*}$ dual to the inclusion $\mathscr{F} \subset T_{X / k}$. Note that $f_{*}(\varphi): f_{*} \mathscr{O}_{X} \rightarrow f_{*}\left(\mathscr{F}^{*}\right)$ is $\mathscr{O}_{Y}$-linear. Indeed, if $y \in \mathscr{O}_{Y}, x \in \mathscr{O}_{X}$ and $s \in \mathscr{F}$ then

$$
(\varphi(y x))(s)=s(y x)=y s(x)+x s(y)=y s(x) .
$$

Therefore we have the following lemma:

LEMMA 2.6. If $f: X \rightarrow Y$ is a quotient by 1 -foliation $\mathscr{F}$ then

$$
0 \rightarrow \mathscr{O}_{Y} \rightarrow f_{*} \mathscr{O}_{X} \rightarrow f_{*}\left(\mathscr{F}^{*}\right)
$$

is an exact sequence of $\mathscr{O}_{Y}$-modules. 
LEMMA 2.7. Let $X$ be a $\mathbb{Q}$-factorial normal variety and let $\mathscr{F} \subset T_{X / k}$ be a 1 -foliation. Then the quotient $Y=X / \mathscr{F}$ is also a $\mathbb{Q}$-factorial normal variety. Moreover, if $X$ is smooth and $D$ is a Weil divisor on $Y$ then pD is Cartier.

Proof. Normality of $Y$ is well-known. Let $D$ be a Weil divisor on $Y$. Let us recall that we have finite morphisms $f: X \rightarrow Y$ and $g: Y \rightarrow X$ such that $f g=F_{Y}$ and $g f=F_{X}$. Since $f^{*} D$ is $\mathbb{Q}$-Cartier and $p D=$ $F_{Y}^{*} D=g^{*}\left(f^{*} D\right)$, the divisor $D$ is also $\mathbb{Q}$-Cartier. If $X$ is smooth then $f^{*} D$ is Cartier, which implies that $p D$ is Cartier.

\subsection{Hirokado's rational vector field}

Let $V$ be a finite dimension $k$-vector space. Let us consider the Euler exact sequence

$$
0 \rightarrow \mathscr{O}_{\mathbb{P}(V)}(-1) \stackrel{s}{\longrightarrow} V^{*} \otimes \mathscr{O}_{\mathbb{P}(V)} \rightarrow T_{\mathbb{P}(V)}(-1) \rightarrow 0,
$$

where $s$ is dual to the evaluation map $V \otimes \mathscr{O}_{\mathbb{P}(V)} \rightarrow \mathscr{O}_{\mathbb{P}(V)}(1)$. Pulling back this sequence by the Frobenius endomorphism Fr: $\mathbb{P}(V) \rightarrow \mathbb{P}(V)$ we have

$$
0 \rightarrow \mathscr{O}_{\mathbb{P}(V)}(-q) \stackrel{\mathrm{Fr}^{*} s}{\longrightarrow} V^{*} \otimes \mathscr{O}_{\mathbb{P}(V)} \rightarrow\left(\mathrm{Fr}^{*} T_{\mathbb{P}(V)}\right)(-q) \rightarrow 0 .
$$

Let us denote by $\theta$ the composition

$$
\mathscr{O}_{\mathbb{P}(V)}(-q) \stackrel{\mathrm{Fr}^{*} s}{\longrightarrow} V^{*} \otimes \mathscr{O}_{\mathbb{P}(V)} \rightarrow T_{\mathbb{P}(V)}(-1)
$$

twisted by the identity map on $\mathscr{O}_{\mathbb{P}(V)}(1)$. This defines a rational vector field on $\mathbb{P}(V)$ with poles of order $(q-1)$. We denote it by $\delta$. This gives a global, coordinate free construction of an analogue of the vector field considered by Hirokado in [13, Proposition 2.1] in case of $\mathbb{P}^{3}$. The following lemma is a generalization of the first part of [13, Proposition 2.1]

LEMMA 2.8. $\delta$ defines a p-closed rational vector field on $\mathbb{P}(V)$ singular along $k$-rational points of $\mathbb{P}(V)$. More precisely, we have $\delta^{[p]}=\delta$.

The proof of this lemma in [13] was omitted but it can be found in the preprint version of the paper.

\section{Determinantal schemes related to Moore's determinant}

Let us recall that a codimension $c$ subscheme of $\mathbb{P}^{n}$ is called standard determinantal if its homogeneous saturated ideal is generated by the $m \times m$ minors of some $m \times(m+c-1)$ homogeneous matrix.

Let $V$ be an $(n+1)$-dimensional vector space over a finite field $k=\mathbb{F}_{q}$, where $q=p^{e}$ for some prime number $p$ and a positive integer $e$. Let us fix some homogeneous coordinates $x_{0}, \ldots, x_{n}$ in $\mathbb{P}(V)$. In the following for a subset $I \subset\{0, \ldots, n\}$ we denote by $|I|$ the number of elements in $I$ and we set $\hat{I}=\{0, \ldots, n\}-$ I. If $|I|=m$ then we order elements of $I$ as $0 \leq i_{1}<\ldots<i_{m} \leq n$ and we write $x_{I}=\left(x_{i_{1}}, \ldots, x_{i_{m}}\right)$.

Proposition 3.1. Let us fix $c \geq 1$ and let $Z_{c} \subset \mathbb{P}(V)$ be the reduced induced subscheme structure on the union of all $k$-linear subspaces of codimension $c$ in $\mathbb{P}(V)$. Then after fixing some homogeneous coordinates $x_{0}, \ldots, x_{n}$ in $\mathbb{P}(V)$ the saturated homogeneous ideal $I\left(Z_{c}\right) \triangleleft k\left[x_{0}, \ldots, x_{n}\right]$ is generated by $\Delta_{q}\left(x_{\hat{I}}\right)$ for all multiindices I such that $|I|=c-1$. Moreover, $Z_{c} \subset \mathbb{P}(V)$ is a standard determinantal scheme. In particular, it is arithmetically Cohen-Macaulay.

Proof. Let us fix some coordinates $x_{0}, \ldots, x_{n}$ in $V^{*}$ that we will also treat as homogeneous coordinates $x_{0}, \ldots, x_{n}$ in $\mathbb{P}(V)$. So we have $V^{*}=\operatorname{Spec} S$ and $\mathbb{P}(V)=$ Proj $S$, where $S=k\left[x_{0}, \ldots, x_{n}\right]$. Let us consider the affine subscheme $Z_{c}^{\prime} \subset V^{*}$ defined by the homogeneous ideal $J \triangleleft S$ generated by the maximal minors of the $(n+2-c) \times(n+1)$ homogeneous matrix

$$
\left(\begin{array}{ccc}
x_{0} & \ldots & x_{n} \\
x_{0}^{q} & \ldots & x_{n}^{q} \\
\vdots & & \vdots \\
x_{0}^{q^{n+1-c}} & & x_{n}^{q^{n+1-c}}
\end{array}\right)
$$


These minors are exactly of the form $\Delta_{q}\left(x_{\hat{I}}\right)$ for multiindices $I$ with $|I|=c-1$. We claim that $Z_{c}^{\prime}$ is a codimension $c$ subscheme, whose underlying set of points is the union of all $k$-linear vector subspaces of codimension $c$ in $V^{*}$.

By definition $Z_{c}^{\prime}$ is the scheme-theoretic intersection of $k$-subschemes $Z_{I}^{\prime} \subset V^{*}$, defined by the ideal generated by $f_{I}:=\Delta_{q}\left(x_{\hat{I}}\right)$ for all multiindices $I$ with $|I|=c-1$. Let us set $P_{i}=\left(0, \ldots, 1^{i}, \ldots, 0\right) \in V^{*}$. By Lemma $2.1 Z_{I}^{\prime}$ is the reduced scheme structure on the set of all $k$-linear hyperplanes passing through 0 and $\left\{P_{i}\right\}_{i \in I}$.

Let $L \subset V^{*}$ be a $k$-vector subspace of codimension $c$. The subspace spanned by $L$ and $0, P_{i_{1}}, \ldots, P_{i_{c-1}}$ is $k$-linear of dimension at most $n$. Therefore it is contained in some $k$-linear hyperplane and hence $L \subset$ $\cap Z_{I}^{\prime}=Z_{c}^{\prime}$. On the other hand, it is clear that if a point $x \in V^{*}$ does not lie on any $k$-vector subspace of $V^{*}$ of codimension $c$, then $x \notin \cap Z_{I}^{\prime}$. Therefore $Z_{c}^{\prime} \subset V^{*}$ has codimension $c$.

Thus we proved that $J$ is a standard determinantal ideal. Therefore by the Eagon-Hochster theorem (or just Eagon's theorem in this case) the ring $S / J$ is Cohen-Macaulay. In particular, $Z_{c}^{\prime}$ has no embedded components. We need to check that $Z_{c}^{\prime}$ is reduced. To do so it is sufficient to check that it is reduced at the generic point of each of its irreducible components. The irreducible components of $Z_{c}^{\prime}$ are codimension $c$ $k$-vector subspaces in $V^{*}$. Let us fix such a subspace $L$. We can choose coordinates so that the ideal of this subspace is equal to $\left(x_{n-c+1}, \ldots, x_{n}\right) \triangleleft S$. We need to check that the Jacobian matrix

$$
J_{\left\{f_{I}\right\}}=\left(\frac{\partial f_{I}}{\partial x_{j}}\right)_{I, j}
$$

has rank $c$ at the generic point of $L$. Let us note that for $n+1-c \leq l \leq n$ we have

$$
\frac{\partial f_{n+1-c, \ldots, \hat{j}, \ldots, n}}{\partial x_{l}}=\frac{\partial \Delta_{q}\left(x_{0}, \ldots, x_{n-c}, x_{j}\right)}{\partial x_{l}}=\left\{\begin{array}{cl}
(-1)^{n+1-c}\left(\Delta_{q}\left(x_{0}, \ldots, x_{n-c}\right)\right)^{q} & \text { for } l=j, \\
0 & \text { for } l \neq j .
\end{array}\right.
$$

Therefore $J_{\left\{f_{I}\right\}}$ contains a $c \times c$ diagonal matrix

$$
\left(\frac{\partial f_{n+1-c, \ldots, \hat{j}, \ldots, n}}{\partial x_{l}}\right)_{n+1-c \leq j, l \leq n}
$$

whose determinant is equal to $(-1)^{c(n+1-c)}\left(\Delta\left(x_{0}, \ldots, x_{n-c}\right)\right)^{c q}$. Since this determinant is non-zero at the generic point of $L, Z_{c}^{\prime}$ is reduced at the generic point of $L$ and thus also reduced. It follows that the graded ideal $J \triangleleft S$ is radical. It is easy to see that graded radical ideals in $S$ different to the ideal $\left(x_{0}, \ldots, x_{n}\right)$ are saturated, so $J \triangleleft S$ is saturated.

It follows that $Z_{c}^{\prime}$ is the affine cone over $Z_{c}$ and $J \triangleleft S$ is also the homogeneous saturated ideal of $Z_{c}$. In particular, $Z_{c}$ is a standard determinantal scheme and it is arithmetically Cohen-Macaulay.

Note that we have a flag of determinantal schemes $Z_{n+1}:=\emptyset \subset Z_{n} \subset \ldots \subset Z_{1} \subset Z_{0}:=\mathbb{P}(V)$, showing that all $Z_{c}$ are so called good determinantal subschemes. Moreover, this flag defines a partition $\mathbb{P}(V)=\bigcup_{i=0}^{n}\left(Z_{i}-Z_{i+1}\right)$, where $\bar{k}$-rational points of $Z_{i}-Z_{i+1}$ are exactly those $x \in(\mathbb{P}(V))(\bar{k})$ for which $x, \operatorname{Fr}(x), \operatorname{Fr}^{2}(x), \ldots$ span a linear subspace of codimension $i$ in $\mathbb{P}(V)$. In particular, this partition is invariant under the Frobenius endomorphism on $\mathbb{P}(V)$.

Let us consider evaluation map ev $: V \otimes \mathscr{O}_{\mathbb{P}(V)} \rightarrow \mathscr{O}_{\mathbb{P}(V)}(1)$ and its pull backs $\left(\mathrm{Fr}^{i}\right)^{*}$ ev $: V \otimes \mathscr{O}_{\mathbb{P}(V)} \rightarrow$ $\mathscr{O}_{\mathbb{P}(V)}\left(q^{i}\right)$ by the Frobenius endomorphisms $\operatorname{Fr}^{i}: \mathbb{P}(V) \rightarrow \mathbb{P}(V)$. Let us fix $c$ ("codimension") and consider the map

$$
g: V \otimes \mathscr{O}_{\mathbb{P}(V)} \rightarrow \mathscr{F}:=\bigoplus_{i=0}^{n+1-c} \mathscr{O}_{\mathbb{P}(V)}\left(q^{i}\right)
$$

given by the direct sum of the above maps.

Proposition 3.1 allows us to write down a free resolution of the homogeneous coordinate $\operatorname{ring}$ of $Z_{c}$. We write down just the corresponding sheafified version of the Eagon-Northcott complex (see [2, Chapter 2.C]). 
COROLlary 3.2. For $1 \leq c \leq n+1$ we have a natural acyclic complex

$$
\begin{aligned}
& 0 \rightarrow \bigwedge^{n+1} V \otimes\left(\operatorname{Sym}^{c-1} \mathscr{F}\right)^{*} \rightarrow \bigwedge^{n} V \otimes\left(\operatorname{Sym}^{c-2} \mathscr{F}\right)^{*} \rightarrow \ldots \rightarrow \bigwedge^{n+3-c} V \otimes\left(\operatorname{Sym}^{1} \mathscr{F}\right)^{*} \rightarrow \\
& \rightarrow \bigwedge^{n+2-c} V \otimes \mathscr{O}_{\mathbb{P}(V)} \rightarrow \mathscr{O}_{\mathbb{P}(V)}\left(1+q+\ldots+q^{n+1-c}\right) \quad \rightarrow \mathscr{O}_{Z_{c}}\left(1+q+\ldots+q^{n+1-c}\right) \rightarrow 0 .
\end{aligned}
$$

In particular, $\bigwedge^{n+2-c} g$ induces an isomorphism

$$
H^{0}\left(\mathbb{P}(V), I_{Z_{c}}\left(\frac{q^{n+2-c}-1}{q-1}\right)\right) \simeq \bigwedge^{n+2-c} V .
$$

By the modular interpretation of the Grassmannian, the map $g$, which is surjective on $\mathbb{P}(V)-Z_{c}$, defines a morphism $\varphi_{c}: \mathbb{P}(V)-Z_{c} \rightarrow \operatorname{Grass}_{k}(V, n+2-c)$. Composing this map with the Plücker embedding Grass $(V, n+2-c) \hookrightarrow \mathbb{P}\left(\bigwedge^{n+2-c} V\right)$, we get the rational map $\mathbb{P}(V)-\rightarrow \mathbb{P}\left(\bigwedge^{n+2-c} V\right)$ defined by the linear system $\left|I_{Z_{c}}\left(1+q+\ldots+q^{n+1-c}\right)\right|$. This immediately follows from Corollary 3.2 and definition of the Plücker embedding. Note that $\left|I_{Z_{c}}\left(1+q+\ldots+q^{n+1-c}\right)\right|$ has scheme theoretic base locus $Z_{c}$ and the map $\mathbb{P}(V)-\rightarrow$ $\mathbb{P}\left(\bigwedge^{n+2-c} V\right)$ is by Lemma 2.3 resolved by the blow up $\mathrm{Bl}_{Z_{c}} \mathbb{P}(V) \rightarrow \mathbb{P}(V)$. After fixing homogenous coordinates in $\mathbb{P}(V)$ this rational map is given by

$$
\left[x_{0}, \ldots, x_{n}\right] \longrightarrow\left[\Delta_{q}\left(x_{I}\right)\right]_{\{I:|I|=n+2-c\}} .
$$

We have canonical isomorphisms $\bigwedge^{i} V \simeq \bigwedge^{n+1-i} V^{*} \otimes \operatorname{det} V$ for $i=0, \ldots, n+1$. They induce canonical isomorphisms $\mathbb{P}\left(\bigwedge^{i} V\right) \simeq \mathbb{P}\left(\bigwedge^{n+1-i} V^{*}\right)$ that are independent of the choice of an orientation $\operatorname{det} V=$ $\bigwedge^{n+1} V \simeq k$. Similarly, we have canonical isomorphisms $\operatorname{Grass}_{k}(V, i) \simeq \operatorname{Grass}_{k}\left(V^{*}, n+1-i\right)$ compatible with the corresponding Plücker embeddings. In fact, this isomorphism can be easily described on the level of functors as sending a quotient $V_{S} \rightarrow \mathscr{E}$ to $V_{S}^{*} \rightarrow\left(\operatorname{ker}\left(V_{S} \rightarrow \mathscr{E}\right)\right)^{*}$.

Therefore we can treat $\varphi_{c}$ as a morphism $\mathbb{P}(V)-Z_{c} \rightarrow \operatorname{Grass}_{k}\left(V^{*}, c-1\right)$. Composing it with the Plücker embedding $\operatorname{Grass}\left(V^{*}, c-1\right) \hookrightarrow \mathbb{P}\left(\bigwedge^{c-1} V^{*}\right)$ we again obtain the map defined by the linear system $\left|I_{Z_{c}}\left(1+q+\ldots+q^{n+1-c}\right)\right|$. In coordinates the corresponding rational map is given by the same equations as before but now the map is written as

$$
\left[x_{0}, \ldots, x_{n}\right] \longrightarrow\left[\Delta_{q}\left(x_{\hat{I}}\right)\right]_{\{I:|I|=c-1\}}
$$

Note that for $c=2$ we get a rational map $\mathbb{P}(V) \rightarrow \mathbb{P}\left(V^{*}\right)$ given by the linear system $\left|I_{Z_{2}}\left(\frac{q^{n}-1}{q-1}\right)\right|$. The study of this rational map is the subject of the following sections.

\section{Linear system of plane curves with moving singularities}

The following proposition generalizes to higher characteristic Serre's example of a linear system with moving singularities in characteristic 2, described in one of exercises in Hartshorne's book (see [12, Chapter III, Exercise 10.7]). It is probably folklore known to some experts or people trying to solve Hartshorne's exercise. It is essentially equivalent to [3, Theorem 4.1] (with a different proof) but it was also known to the author earlier. We describe this example separately as it is an elementary special case of the more general linear system used later in Section 5 .

PROPOSITION 4.1. The linear system $\left|I_{Z}(q+1)\right|$ of degree $(q+1)$ plane curves on $\mathbb{P}^{2}$ containing $Z=$ $\mathbb{P}^{2}\left(\mathbb{F}_{q}\right)$ has dimension 2 with base points $Z$. It determines an inseparable degree $p$ morphism from $\mathbb{P}^{2}-Z$ to $\mathbb{P}^{2}$, which as a rational map $\mathbb{P}^{2} \rightarrow \mathbb{P}^{2}$ is resolved by blowing up $Z$. Moreover, on $\mathbb{P}_{\bar{k}}^{2}$ every curve $C \in\left|I_{Z}(q+1)\right|$ is singular at exactly one point of $\mathbb{P}^{2}(\bar{k})$ determining a bijection between curves in $\left|I_{Z}(q+1)\right|$ and $\mathbb{P}^{2}(\bar{k})$.

Proof. The map $\theta$ from Subsection 2.6 gives rise to the short exact sequence

$$
0 \rightarrow \mathscr{O}_{\mathbb{P}^{2}}(-q+1) \stackrel{\theta}{\rightarrow} T_{\mathbb{P}^{2}} \rightarrow I_{Z}(q+2) \rightarrow 0 .
$$


Twisting this sequence by $\mathscr{O}_{\mathbb{P}^{2}}(-1)$ we get

$$
0 \rightarrow \mathscr{O}_{\mathbb{P}^{2}}(-q) \rightarrow T_{\mathbb{P}^{2}}(-1) \rightarrow I_{Z}(q+1) \rightarrow 0
$$

Therefore we get $h^{0}\left(I_{Z}(q+1)\right)=3$. A basis of $H^{0}\left(\mathbb{P}^{2}, I_{Z}(q+1)\right)$ can be obtained by fixing three distinct points of $Z$ and taking products of equations of all $\mathbb{F}_{q}$-rational lines passing through the fixed points. Let us recall that the group $\mathbb{F}_{q}^{*}$ is cyclic and hence

$$
x_{1} \prod_{a \in \mathbb{F}_{q}}\left(x_{2}-a x_{1}\right)=x_{1} x_{2}^{q}-x_{1}^{q} x_{2}
$$

(this is also a special case of Lemma 2.1). So for example taking $[1,0,0],[0,1,0]$ and $[0,0,1]$, we see that $s_{0}=x_{1} x_{2}^{q}-x_{1}^{q} x_{2}, s_{1}=x_{0}^{q} x_{2}-x_{0} x_{2}^{q}$ and $s_{2}=x_{0} x_{1}^{q}-x_{0}^{q} x_{1}$ form a basis of $H^{0}\left(\mathbb{P}^{2}, I_{Z}(q+1)\right)$.

Let us fix $a, b, c \in \bar{k}$, not all equal to 0 , and consider the polynomial

$$
f\left(x_{0}, x_{1}, x_{2}\right):=a s_{0}+b s_{1}+c s_{2} .
$$

Since

$$
\frac{\partial f}{\partial x_{0}}=-b x_{2}^{q}+c x_{1}^{q}, \quad \frac{\partial f}{\partial x_{1}}=a x_{2}^{q}-c x_{0}^{q}, \quad \frac{\partial f}{\partial x_{2}}=b x_{0}^{q}-a x_{1}^{q},
$$

the point $P=[\sqrt[q]{a}, \sqrt[q]{b}, \sqrt[q]{c}] \in \mathbb{P}^{2}(\bar{k})$ is the only singular point of the curve $f\left(x_{0}, x_{1}, x_{2}\right)=0$.

Assume that $a \neq 0$ and let us set

$$
y_{1}=\frac{x_{1}}{x_{0}}-\sqrt[q]{\frac{b}{a}} \quad \text { and } \quad y_{2}=\frac{x_{2}}{x_{0}}-\sqrt[q]{\frac{c}{a}}
$$

Then in the corresponding affine chart, the curve $f\left(x_{0}, x_{1}, x_{2}\right)=0$ has equation

$$
\left(\sqrt[q]{\frac{c}{a}}-\frac{c}{a}\right) y_{1}^{q}+\left(\sqrt[q]{\frac{b}{a}}-\frac{b}{a}\right) y_{2}^{q}+y_{1} y_{2}^{q}-y_{1}^{q} y_{2}=0
$$

Therefore if $[a, b, c] \in \mathbb{P}^{2}\left(\mathbb{F}_{q}\right)$ then in this chart the curve $f\left(x_{0}, x_{1}, x_{2}\right)=0$ is given by $y_{1} y_{2}^{q}-y_{1}^{q} y_{2}=0$. So the curve $f\left(x_{0}, x_{1}, x_{2}\right)=0$ consists of all $\mathbb{F}_{q}$-rational lines passing through the point $P$ and $P=[a, b, c]$. If $[a, b, c] \notin \mathbb{P}^{2}\left(\mathbb{F}_{q}\right)$ then $P$ is a singular point of $f\left(x_{0}, x_{1}, x_{2}\right)=0$ of multiplicity $q$ with only 1 tangent direction.

Let us consider the Frobenius morphism $\mathrm{Fr}_{\mathbb{P}^{2}}: \mathbb{P}^{2} \rightarrow \mathbb{P}^{2}$ given by $\left[x_{0}, x_{1}, x_{2}\right] \rightarrow\left[x_{0}^{q}, x_{1}^{q}, x_{2}^{q}\right]$. Note that by definition $P$ is the preimage of $[a, b, c]$. If $P$ is a fixed point of $\operatorname{Fr}_{\mathbb{P}^{2}}$ then the curve $f\left(x_{0}, x_{1}, x_{2}\right)=0$ consists of $(q+1)$ lines passing through $P$. Otherwise, $f\left(x_{0}, x_{1}, x_{2}\right)=0$ has only one singularity of multiplicity $q$ at $P$. This shows that the correspondence sending $C \in\left|I_{Z}(q+1)\right|$ to the singular point of $C$ is a bijection and the singular points of elements of the linear system $\left|I_{Z}(q+1)\right|$ move all over $\mathbb{P}^{2}(\bar{k})$.

Remark 4.2. Note that a part of [12, Chapter III, Exercise 10.7] is incorrect. Namely, the curves $f\left(x_{0}, x_{1}, x_{2}\right)=$ 0 with singularities outside of $Z$ can be reducible (even in characteristic 2 ).

For example, consider the curve $C:=\left(f\left(x_{0}, x_{1}, x_{2}\right)=0\right)$ with singularity at a point $Q \notin Z$ but lying on a line $L$ defined over $\mathbb{F}_{q}$. Note that $C$ contains at least $(q+2)$ points of $L$. Namely, it contains point $Q$ and $(q+1)$ points $L\left(\mathbb{F}_{q}\right)$. Since $C$ has degree $(q+1)$, it must contain $L$ and hence $D$ is reducible. More precisely $C=L+C^{\prime}$, where $C^{\prime}$ is a degree $q$ curve passing through $Z-L$ and having multiplicity $(q-1)$ at $Q$ (it is easy to see that at $Q$, the curve $C^{\prime}$ is of type $\alpha y_{1}^{q-1}-y_{1}^{q-1} y_{2}+y_{2}^{q}=0$ for some $\alpha \neq 0$ ). Moreover, $C^{\prime}$ intersects $L$ at exactly one point $Q$ as any other intersection point would give another singularity of $C$. In characteristic 2 the corresponding cubic curve $C$ consists of the line $L$ and a smooth conic $C^{\prime}$ tangent to it at $Q$. 


\section{Purely inseparable endomorphisms of Drinfeld half-spaces}

As before, let $V$ be an $(n+1)$-dimensional vector space over a finite field $k=\mathbb{F}_{q}$, where $q=p^{e}$ for some prime number $p$ and a positive integer $e$. Let $\Omega(V)$ be the Drinfeld half-space, i.e., the complement of all $k$-rational hyperplanes in $\mathbb{P}(V)$. In the following we assume that $n \geq 1$ as everything is trivial if $\operatorname{dim} V \leq 1$.

Let us consider Euler's exact sequence

$$
0 \rightarrow \mathscr{O}_{\mathbb{P}(V)}(-1) \stackrel{s}{\rightarrow} V^{*} \otimes \mathscr{O}_{\mathbb{P}(V)} \rightarrow T_{\mathbb{P}(V)}(-1) \rightarrow 0
$$

where $s$ is the dual of the evaluation map $V \otimes \mathscr{O}_{\mathbb{P}(V)} \rightarrow \mathscr{O}_{\mathbb{P}(V)}(1)$. This gives us a canonical embedding $\mathbb{P}\left(T_{\mathbb{P}(V)}\right) \subset \mathbb{P}(V) \times_{k} \mathbb{P}\left(V^{*}\right)$. Pulling back the above sequence by the composition of Frobenius endomorphisms $\operatorname{Fr}^{i}: \mathbb{P}(V) \rightarrow \mathbb{P}(V)$ we obtain

$$
0 \rightarrow \mathscr{O}_{\mathbb{P}(V)}\left(-q^{i}\right) \stackrel{\left(\mathrm{Fr}^{i}\right)^{*} s}{\longrightarrow} V^{*} \otimes \mathscr{O}_{\mathbb{P}(V)} \rightarrow\left(\left(\mathrm{Fr}^{i}\right)^{*} T_{\mathbb{P}(V)}\right)\left(-q^{i}\right) \rightarrow 0
$$

So we also have canonical embeddings $\mathbb{P}\left(\left(\mathrm{Fr}^{i}\right)^{*} T_{\mathbb{P}(V)}\right) \subset \mathbb{P}(V) \times_{k} \mathbb{P}\left(V^{*}\right)$.

Let $X_{V}$ be the scheme theoretic intersection of $\bigcap_{i=0}^{n-1} \mathbb{P}\left(\left(\mathrm{Fr}^{i}\right)^{*} T_{\mathbb{P}(V)}\right) \subset \mathbb{P}(V) \times{ }_{k} \mathbb{P}\left(V^{*}\right)$. Projections of $X_{V}$ onto the first and second factor are denoted by $\pi_{1, V}$ and $\pi_{2, V}$, respectively.

In the following we denote by $\mathscr{L}(V)$ the set of all proper $k$-linear subspaces of $\mathbb{P}(V)$. Every $k$-linear subspace $L \in \mathscr{L}(V)$ is of the form $\mathbb{P}(V / W)$ for some $k$-linear subspace $W \subset V$. For $L=\mathbb{P}(V / W) \subsetneq \mathbb{P}(V)$ we set $L^{\perp}=\mathbb{P}\left(W^{*}\right) \subsetneq \mathbb{P}\left(V^{*}\right)$. If $\eta_{L}$ denotes the generic point of $L$ then we set $E_{L}:=\overline{\pi_{1, V}^{-1}\left(\eta_{L}\right)} \subset X_{V}$.

LEMMA 5.1. For any $L \in \mathscr{L}(V)$ we have $E_{L}=L \times_{k} L^{\perp}$ and $\pi_{1, V}^{-1}(L)=\bigcup_{L^{\prime} \in \mathscr{L}(V), L^{\prime} \subset L} E_{L^{\prime}}$. In particular, $E_{L} / k$ is smooth of dimension $(n-1)$ and $\pi_{1, V}^{-1}(L)$ is pure of dimension $(n-1)$.

Proof. In the following $[v] \in \mathbb{P}(V)(\bar{k})$ denotes the $\bar{k}$-rational point determined by $\bar{k}$-point $v \in V^{*}(\bar{k})$. Similarly, $[w] \in \mathbb{P}\left(V^{*}\right)(\bar{k})$ denotes the $\bar{k}$-point determined by $w \in V(\bar{k})=\left(V^{*}\right)^{*}(\bar{k})$. Let us note that $X_{V}(\bar{k}) \subset$ $\mathbb{P}(V)(\bar{k}) \times \mathbb{P}\left(V^{*}\right)(\bar{k})$ is equal to

$$
\left\{([v],[w]):\langle v, w\rangle=\langle\operatorname{Fr}(v), w\rangle=\ldots=\left\langle\operatorname{Fr}^{n-1}(v), w\right\rangle=0\right\}
$$

where $\langle\cdot, \cdot\rangle$ denotes the natural pairing $V^{*}(\bar{k}) \times V(\bar{k}) \rightarrow \bar{k} . \bar{k}$-rational points of $\mathbb{P}(V)$ correspond to hyperplanes in $V_{\bar{k}}$ and a subspace $W \subset V_{\bar{k}}$ is $k$-rational if and only if $\operatorname{Fr}(W)=W$. So for a $k$-rational subspace $L \subset \mathbb{P}(V)$ we have $([v],[w]) \in \pi_{1, V}^{-1}(L)(\bar{k})$ if $[v] \in L(\bar{k})$ and $[w] \in L^{\perp}(\bar{k})$. Note that if $L \subsetneq \mathbb{P}(V)$ has codimension $c$ then $L^{\perp} \subsetneq \mathbb{P}\left(V^{*}\right)$ has dimension $(c-1)$. Therefore if $\eta_{L}$ denotes the generic point of $L$ then

$$
E_{L}=\overline{\pi_{1, V}^{-1}\left(\eta_{L}\right)}=\overline{\pi_{1, V}^{-1}\left(L-\bigcup_{L^{\prime} \in \mathscr{L}(V), L^{\prime} \subsetneq L} L^{\prime}\right)}=L \times_{k} L^{\perp}
$$

is a smooth divisor in $X$ and $\pi_{2, V}\left(\pi_{1, V}^{-1}\left(\eta_{L}\right)\right)$ is the generic point of the $k$-rational subspace $L^{\perp} \subsetneq \mathbb{P}\left(V^{*}\right)$.

From now on to simplify notation we denote $Z_{2}$ from Proposition 3.1 by $Z$.

PROPOSITION 5.2. The k-scheme $X_{V}$ has the following properties.

1. $X_{V}$ is geometrically integral.

2. $X_{V}$ has dimension $n$. In particular, $X_{V}$ is a complete intersection in $\mathbb{P}(V) \times_{k} \mathbb{P}\left(V^{*}\right)$.

3. $\pi_{1, V}: X_{V} \rightarrow \mathbb{P}(V)$ is the blow up of $\mathbb{P}(V)$ along $Z$.

4. The singular locus of $X_{V} / k$ is a divisor equal to $\operatorname{Sing} X_{V}=\bigcup_{L \in \mathscr{L}(V), \operatorname{codim} L \geq 3} E_{L}$.

5. $X_{V}$ is smooth if $n=2$ and $X_{V}$ is non-normal if $n \geq 3$.

6. $X_{V}$ is the graph of the rational map $\psi_{V}: \mathbb{P}(V) \rightarrow \mathbb{P}\left(V^{*}\right)$. 
Proof. Let us fix homogeneous coordinates $x_{0}, \ldots, x_{n}$ in $\mathbb{P}(V)$ and dual homogeneous coordinates $y_{0}, \ldots, y_{n}$ in $\mathbb{P}\left(V^{*}\right)$. Then $X_{V}$ is defined by the ideal in the bigraded ring $k\left[x_{0}, \ldots, x_{n} ; y_{0}, \ldots, y_{n}\right]$ generated by bihomogeneous polynomials $f_{0}=x_{0} y_{0}+\ldots+x_{n} y_{n}, f_{1}=x_{0}^{q} y_{0}+\ldots+x_{n}^{q} y_{n}, \ldots, f_{n-1}=x_{0}^{q^{n-1}} y_{0}+\ldots+x_{n}^{q^{n-1}} y_{n}$. To check where $X_{V} \rightarrow$ Spec $k$ is smooth let us consider the Jacobian matrix

$$
\left(\frac{\partial f_{i}}{\partial x_{j}}, \frac{\partial f_{i}}{\partial y_{j}}\right)_{i=0, \ldots, n-1, j=0,1, \ldots, n}=\left(\begin{array}{cccccc}
y_{0} & \ldots & y_{n} & x_{0} & \ldots & x_{n} \\
0 & \ldots & 0 & x_{0}^{q} & \ldots & x_{n}^{q} \\
\vdots & & \vdots & \vdots & & \vdots \\
0 & \ldots & 0 & x_{0}^{q^{n-1}} & \ldots & x_{n}^{q^{n-1}}
\end{array}\right) .
$$

The only non-zero $n$th order minors of this matrix (up to a sign) are of the form

$$
\Delta_{q}\left(x_{0}, \ldots, \hat{x}_{i}, \ldots, x_{n}\right) \quad \text { and } \quad y_{i} \cdot\left(\Delta_{q}\left(x_{0}, \ldots, \hat{x}_{i}, \ldots, \hat{x_{j}}, \ldots, x_{n}\right)\right)^{q}
$$

for $i, j=1, \ldots, n$ such that $i \neq j$. Let $x$ be a point of the subscheme of $X_{V}$ defined by these minors. Since for some $i$ we have $y_{i}(x) \neq 0, x$ lies in the preimage under $\pi_{1, V}$ of the subscheme of $\mathbb{P}(V)$ defined by the ideal generated by $\Delta_{q}\left(x_{0}, \ldots, \hat{x}_{i}, \ldots, \hat{x}_{j}, \ldots, x_{n}\right)$ for $i \neq j$. By Proposition 3.1 we have $x \in \pi_{1, V}^{-1}\left(\bigcup_{L \in \mathscr{L}(V), \operatorname{codim} L=3} L\right)$. Therefore the above Jacobian matrix has rank $n$ precisely at the points not lying over a $k$-linear subspace of $\mathbb{P}(V)$ of codimension 3. It follows that $\pi_{1, V}^{-1}\left(\mathbb{P}(V)-\bigcup_{L \in \mathscr{L}(V) \text {, codim } L=3} L\right)$ is smooth of dimension $n$ over $k$ and the singular locus of $X_{V} / k$ equals to $\pi_{1, V}^{-1}\left(\bigcup_{L \in \mathscr{L}(V), \operatorname{codim} L=3} L\right)=\bigcup_{L \in \mathscr{L}(V), \operatorname{codim} L \geq 3} E_{L}$.

Expansion with respect to the last row gives

$$
0=\operatorname{det}\left(\begin{array}{ccc}
x_{0} & \ldots & x_{n} \\
\vdots & & \vdots \\
x_{0}^{q^{n-1}} & \ldots & x_{n}^{q^{n-1}} \\
x_{0}^{q^{j}} & \ldots & x_{n}^{q^{j}}
\end{array}\right)=\sum(-1)^{n+i} x_{i}^{q^{j}} \Delta_{q}\left(x_{0}, \ldots, \hat{x}_{i}, \ldots, x_{n}\right)
$$

for $j=0, \ldots, n-1$. Therefore $y_{i}-(-1)^{n+i} \Delta_{q}\left(x_{0}, \ldots, \hat{x}_{i}, \ldots, x_{n}\right) \in I(X)$ for $i=0, \ldots, n$ proving that the projection $\pi_{1, V}: X_{V} \rightarrow \mathbb{P}(V)$ is surjective. Since the fibers of $\left(\pi_{1, V}\right)_{\bar{k}}:\left(X_{V}\right)_{\bar{k}} \rightarrow \mathbb{P}(V)_{\bar{k}}$ are linear subspaces of $\mathbb{P}(V)_{\bar{k}}$, it follows that $X_{V}$ is geometrically connected.

Now we claim that $\pi_{1, V}^{\prime}:=\left.\pi_{1, V}\right|_{X_{V}-\pi_{1, V}^{-1}(Z)}: X_{V}-\pi_{1, V}^{-1}(Z) \rightarrow \mathbb{P}(V)-Z$ is an isomorphism. To prove that it is sufficient to check that $\pi_{1, V}^{\prime}$ is smooth and bijective on points.

Let us take $x \in \mathbb{P}(V)-Z$. Then there exists $i$ such that $x \in D_{+}\left(\Delta_{q}\left(x_{0}, \ldots, \hat{x}_{i}, \ldots, x_{n}\right)\right)$. We claim that $\pi_{1, V}^{-1}(x) \subset \pi_{2, V}^{-1}\left(D_{+}\left(y_{i}\right)\right)$. To prove that it is sufficient to note that the system of linear equations (in $\left.y_{0}, \ldots, \hat{y}_{i}, \ldots y_{n}\right)$

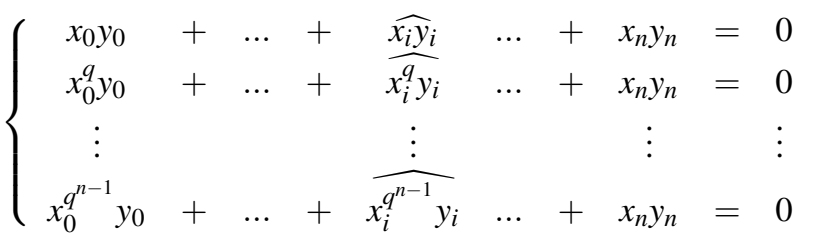

has no non-zero solutions. If we consider $\pi_{2, V}^{-1}\left(D_{+}\left(y_{i}\right)\right)$ then $\pi_{1, V}^{-1}(x)$ is defined by the system of linear equations

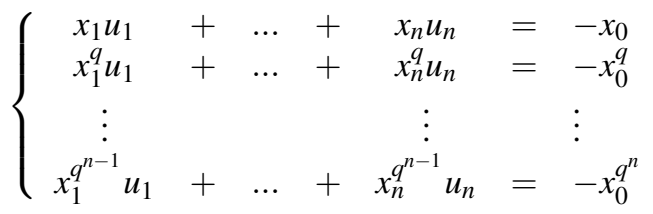

which has exactly one solution. Corollary 3.1 implies that the union of all $k$-linear subspaces of codimension 2 is given by vanishing of $\Delta_{q}\left(x_{0}, \ldots, \hat{x}_{i}, \ldots, x_{n}\right)$ for $i=0, \ldots, n$. Therefore the restriction of $\pi_{1}$ to the 
preimage of the union of all $k$-linear subspaces of codimension 2 is an isomorphism with the inverse given by $x \rightarrow(x, \psi(x))$, where $\psi: \mathbb{P}(V)-Z \rightarrow \mathbb{P}\left(V^{*}\right)=\operatorname{Proj} k\left[y_{0}, \ldots, y_{n}\right]$ is given by

$$
\psi^{*}\left(y_{i}\right)=(-1)^{i} \Delta_{q}\left(x_{0}, \ldots, \hat{x}_{i}, \ldots, x_{n}\right) .
$$

In particular, by Section 3 the map $\psi$ is given by the linear system $\left|I_{Z}\left(1+q+\ldots+q^{n-1}\right)\right|$.

Since the ideal of $X_{V}$ is generated by $n$ elements, each irreducible component of $X_{V}$ has dimension $\geq \operatorname{dim} \mathbb{P}(V) \times_{k} \mathbb{P}\left(V^{*}\right)-n=n$. On the other hand, $X_{V}-\pi_{1, V}^{-1}(Z)$ is irreducible and by Lemma $5.1 \pi_{1, V}^{-1}(Z)$ is pure of dimension $n-1$, so $X_{V}$ is irreducible. In fact, the same arguments work also for $\left(X_{V}\right)_{\bar{k}}$, so $X_{V}$ is geometrically irreducible. Since $X_{V}$ is smooth at the generic point and it is locally a complete intersection, it is also geometrically reduced. Therefore $X_{V}$ is geometrically integral and by the above it is equal to the graph of $\psi: \mathbb{P}(V) \rightarrow \mathbb{P}\left(V^{*}\right)$. Then Lemma2.3 implies that $X_{V}$ is the blow up of $\mathbb{P}(V)$ along $Z$.

Proposition 5.3. There exists a finite, purely inseparable morphism $\varphi_{X_{V}}: X_{V} \rightarrow X_{V^{*}}$ such that the diagram

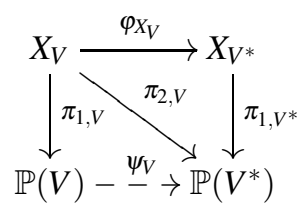

is commutative. Moreover, we have $\varphi_{X_{V^{*}}} \circ \varphi_{X_{V}}=\mathrm{Fr}_{X_{V}}^{n-1}$. The restriction of $\psi_{V}$ to $\Omega(V)$ gives a finite purely

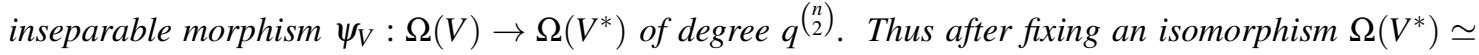
$\Omega(V)$, we get a purely inseparable endomorphism of Drinfeld's half-space $\Omega(V)$.

Proof. Let $p_{1}$ and $p_{2}$ be the projections of $\mathbb{P}(V) \times_{k} \mathbb{P}\left(V^{*}\right)$ onto the first and second factor, respectively. Let us consider the map $\bar{\varphi}_{V}: \mathbb{P}(V) \times_{k} \mathbb{P}\left(V^{*}\right) \rightarrow \mathbb{P}\left(V^{*}\right) \times_{k} \mathbb{P}(V)$ defined by

$$
\bar{\varphi}_{V}=p_{2} \times_{k}\left(\operatorname{Fr}_{\mathbb{P}(V)}^{n-1} \circ p_{1}\right) .
$$

After fixing projective coordinates $\bar{\varphi}_{V}$ is given by

$$
\left(\left[x_{0}, \ldots, x_{n}\right],\left[y_{0}, \ldots, y_{n}\right]\right) \rightarrow\left(\left[v_{0}, \ldots, v_{n}\right],\left[w_{0}, \ldots, w_{n}\right]\right)=\left(\left[y_{0}, \ldots, y_{n}\right],\left[x_{0}^{q^{n-1}}, \ldots, x_{n}^{q^{n-1}}\right]\right) .
$$

Since

$$
\left(\sum x_{i}^{q^{j}} y_{i}\right)^{q^{n-1-j}}=\sum x_{i}^{q^{n-1}} y_{i}^{q^{n-1-j}}=\sum v_{i}^{q^{n-1-j}} w_{i}
$$

for $j=0,1, \ldots, n-1, \bar{\varphi}_{V}$ maps $\mathbb{P}\left(\left(\mathrm{Fr}^{j}\right)^{*} T_{\mathbb{P}(V)}\right)$ into $\mathbb{P}\left(\left(\mathrm{Fr}^{n-1-j}\right)^{*} T_{\mathbb{P}\left(V^{*}\right)}\right)$ and therefore $X_{V}$ into $X_{V^{*}}$. We define $\varphi_{X_{V}}$ as the restriction $\left.\bar{\varphi}_{V}\right|_{X}: X_{V} \rightarrow X_{V^{*}}$.

Let us note that $\pi_{2, V}=\pi_{1, V^{*}} \circ \varphi_{X_{V}}$. Moreover, since $\bar{\varphi}_{V^{*}} \circ \bar{\varphi}_{V}=\operatorname{Fr}_{\mathbb{P}(V) \times_{k} \mathbb{P}(V)}^{n-1}$, we have $\varphi_{X_{V^{*}}} \circ \varphi_{X_{V}}=$ $\operatorname{Fr}_{X_{V}}^{n-1}$. In particular, $\varphi_{X_{V}}$ is a purely inseparable map of degree $q^{\left(\begin{array}{c}n \\ 2\end{array}\right) \text {. }}$

We need to prove that $\psi_{V}$ maps $\Omega(V)$ into $\Omega\left(V^{*}\right)$. Since $\pi_{1, V}: X_{V} \rightarrow \mathbb{P}(V)$ is the blow up of $\mathbb{P}(V)$ along $Z$ and Lemma 2.1 implies that $\Omega(V)$ is contained in $\mathbb{P}(V)-Z,\left.\pi_{1, V}\right|_{\pi_{1, V}^{-1}(\Omega(V))}: \pi_{1, V}^{-1}(\Omega(V)) \rightarrow \Omega(V)$ is an isomorphism. So we can treat $X_{V}$ as a compactification of Drinfeld's half-space $\Omega(V)$. Since by Lemma 5.1

$$
\pi_{1, V}^{-1}(\Omega(V))=X_{V}-\bigcup_{L \in \mathscr{L}(V)} E_{L}
$$

and $\varphi_{X_{V}}$ maps $E_{L}$ into $E_{L^{\perp}}, \varphi_{X_{V}}$ maps $\pi_{1, V}^{-1}(\Omega(V))$ into $\pi_{1, V^{*}}^{-1}\left(\Omega\left(V^{*}\right)\right)$. But $\left.\psi_{V}\right|_{\mathbb{P}(V)-Z}=\pi_{1, V^{*}} \circ \varphi_{X_{V}} \circ$ $\left(\pi_{1, V} \mid \mathbb{P}(V)-Z\right)^{-1}$, so $\psi_{V}(\Omega(V)) \subset \Omega\left(V^{*}\right)$. Since $\varphi_{X_{V}}$ is finite and purely inseparable, $\psi_{V}: \Omega(V) \rightarrow \Omega\left(V^{*}\right)$ is also finite and purely inseparable. Moreover, since $\varphi_{X_{V^{*}}} \circ \varphi_{X_{V}}=\operatorname{Fr}_{X}^{n-1}$ we have $\psi_{V^{*}} \circ \psi_{V}=\operatorname{Fr}_{\Omega(V)}^{n-1}$.

Proposition 5.4. The projection $\pi_{2, V}: X_{V} \rightarrow \mathbb{P}\left(V^{*}\right)$ onto the second factor decomposes into a birational morphism $f_{V}: X_{V} \rightarrow Y_{V}$ and a purely inseparable morphism $\varphi_{Y_{V}}: Y_{V} \rightarrow \mathbb{P}\left(V^{*}\right)$. Morphism $f_{V}$ is an isomorphism outside of $\left(\bigcup_{L \in \mathscr{L}(V), \operatorname{dim} L>0} E_{L}\right)$ and we have $\operatorname{codim} E_{L}=1$ and $\operatorname{dim} f\left(E_{L}\right)=\operatorname{codim} L-1$. 
Proof. Let us consider Stein's factorization $X_{V} \rightarrow Y_{V} \rightarrow \mathbb{P}\left(V^{*}\right)$ of $\pi_{2, V}$. In particular, $f_{V}: X_{V} \rightarrow Y_{V}$ is birational with connected fibers and $\varphi_{Y_{V}}: Y_{V} \rightarrow \mathbb{P}(V)$ is finite. Note that

$$
\left.\pi_{2, V}\right|_{\pi_{1, V}^{-1}(\Omega(V))}: \pi_{1, V}^{-1}(\Omega(V)) \stackrel{\sim}{\rightarrow} \Omega(V) \stackrel{\psi_{V}}{\rightarrow} \Omega\left(V^{*}\right)
$$

is finite and purely inseparable, so $\varphi_{Y_{V}}$ is purely inseparable.

Clearly, we have $\varphi_{X_{V}}\left(E_{L}\right)=E_{L^{\perp}}$. Since $E_{L}$ is defined over $k$, we have $\operatorname{Fr}_{X}^{-1}\left(E_{L}\right)=E_{L}$ and hence $\varphi_{X_{V}}^{-1}\left(E_{L}\right)=E_{L}$. Note that

$$
\pi_{2, V}^{-1}(Z)=\varphi_{X_{V}}^{-1}\left(\pi_{1, V}^{-1}(Z)\right)=\varphi_{X_{V}}^{-1}\left(\bigcup_{L \in \mathscr{L}(V), \operatorname{codim} L \geq 2} E_{L}\right)=\bigcup_{L \in \mathscr{L}(V), \operatorname{codim} L \geq 2} E_{L^{\perp}}=\bigcup_{L \in \mathscr{L}(V), \operatorname{dim} L>0} E_{L}
$$

and $f_{V}$ is an isomorphism on $\pi_{2, V}^{-1}(\mathbb{P}(V)-Z)$. Therefore $\operatorname{Exc}\left(f_{V}\right)=\bigcup_{L \in \mathscr{L}(V), \operatorname{dim} L>0} E_{L}$.

Remark 5.5. It is easy to see that $Y_{V}$ is a normal projective $k$-variety. Indeed, there exists a birational morphism from the wonderful compactification $\bar{X} \rightarrow X_{V}$ (cf. Section 6) and $\bar{X} \rightarrow Y_{V} \rightarrow \mathbb{P}\left(V^{*}\right)$ is Stein's factorization, so $Y_{V}$ is normal.

THEOREM 5.6. There exists a finite morphism $\varphi_{V}: \mathbb{P}(V) \rightarrow Y_{V^{*}}$ such that we have the following commutative diagram of $k$-schemes

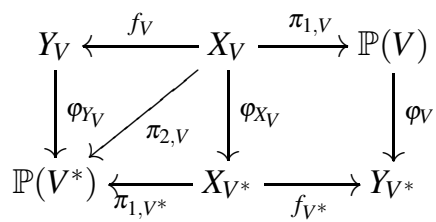

in which horizontal maps are birational, vertical maps are purely inseparable and they satisfy the following relations

$$
\varphi_{X_{V^{*}}} \circ \varphi_{X_{V}}=\operatorname{Fr}_{X_{V}}^{\operatorname{dim} V-2}, \quad \varphi_{Y_{V^{*}}} \circ \varphi_{V}=\operatorname{Fr}_{\mathbb{P}(V)}^{\operatorname{dim} V-2}, \quad \varphi_{V^{*}} \circ \varphi_{Y_{V}}=\operatorname{Fr}_{Y_{V}}^{\operatorname{dim} V-2}
$$

The rational map $\psi_{V}:=\pi_{1, V^{*}} \varphi_{X_{V}} \pi_{1, V}^{-1}: \mathbb{P}(V) \rightarrow \mathbb{P}\left(V^{*}\right)$ is purely inseparable and it satisfies $\psi_{V^{*}} \circ \psi_{V}=$ $\operatorname{Fr}_{\mathbb{P}(V)}^{\operatorname{dim} V-2}$. Moreover, $\psi_{V}$ defines a finite morphism $\psi_{V}: \Omega(V) \rightarrow \Omega\left(V^{*}\right)$ such that $\psi_{V^{*}} \circ \psi_{V}=\operatorname{Fr}_{\Omega(V)}^{\operatorname{dim} V-2}$.

Proof. Let us consider Stein's factorization of the map $f_{V^{*}} \circ \varphi_{X_{V}}: X_{V} \rightarrow Y_{V^{*}}$. We get a proper morphism $g_{1}: X_{V} \rightarrow Y^{\prime}$ such that $\left(g_{1}\right)_{*} \mathscr{O}_{X_{V}}=\mathscr{O}_{Y^{\prime}}$ and a finite affine morphism $g_{2}: Y^{\prime} \rightarrow Y_{V^{*}}$. Now let us consider the morphism $h: X_{V} \rightarrow \mathbb{P}(V)$ defined by $h:=\operatorname{Fr}_{\mathbb{P}(V)}^{\operatorname{dim} V-2} \circ \pi_{1, V}$. Note that $h_{2}=\varphi_{Y_{V^{*}}} \circ g_{2}$ is finite and affine, so we have two Stein's factorizations of $h$, one coming from the definition and another one given by $h=h_{2} \circ g_{1}$. It follows that $Y^{\prime}$ and $\mathbb{P}(V)$ are isomorphic as affine schemes over $\mathbb{P}(V)$ (as they are spectrums of the same sheaf of algebras $\left.h_{*} \mathscr{O}_{\mathbb{P}(V)}\right)$ and the birational map $g_{1} \circ \pi_{1, V}^{-1}: \mathbb{P}(V) \rightarrow Y^{\prime}$ extends to an isomorphism. It follows that the birational map $\varphi_{V}:=f_{V^{*}} \circ \varphi_{X_{V}} \circ \pi_{1, V}^{-1}: \mathbb{P}(V)-\rightarrow Y_{V^{*}}$ extends to a morphism.

The remaining assertions follow easily from Propositions 5.2, 5.3 and 5.4

\section{Wonderful compactification of Drinfeld's half-space}

As before, let $V$ be an $(n+1)$-dimensional vector space over a finite field $k=\mathbb{F}_{q}$ and let $\mathscr{L}(V)$ be the set of all proper $k$-linear subspaces of $\mathbb{P}(V) . \mathscr{L}(V)$ forms an arrangement of subvarieties of $\mathbb{P}(V)$ and so we can consider the wonderful compactification $\tilde{X}$ of $\Omega(V)$. By Theorem 2.5 we can construct $\tilde{X}$ by successively blowing up strict transforms of $k$-linear subspaces of $\mathbb{P}(V)$ of increasing dimensions. So we have

$$
\tilde{X}=\tilde{X}_{n} \stackrel{\tilde{\pi}_{n-1}}{\longrightarrow} \tilde{X}_{n-1} \longrightarrow \cdots \longrightarrow \tilde{X}_{2} \stackrel{\tilde{\pi}_{1}}{\longrightarrow} \tilde{X}_{1} \stackrel{\tilde{\pi}_{0}}{\longrightarrow} \tilde{X}_{0}=\mathbb{P}(V)
$$

where $\tilde{\pi}_{i}$ is the blow up of $\tilde{X}_{i}$ along the strict transforms of $k$-linear subspaces of $\mathbb{P}(V)$ of dimension $i$ (in particular $\tilde{\pi}_{n-1}$ is an isomorphism). The canonical map $\tilde{X} \rightarrow \mathbb{P}(V)$ is denoted by $\tilde{\pi}$. The pull-back of the hyperplane section to $\tilde{X}$ is denoted by $H$. 
Note that by Lemma 2.4 we have canonical morphisms $\tilde{X}_{i} \rightarrow \mathrm{Bl}_{Z_{c}} \mathbb{P}(V)$ for $c=n+1-i, \ldots, n+1$. Therefore Corollary 3.2 implies that for $c=n+1-i, \ldots, n+1$ the linear system $\left|I_{Z_{c}}\left(1+q+\ldots+q^{n+1-c}\right)\right|$ becomes base point free on $\tilde{X}_{i}$ giving rise to the canonical morphism

$$
\varphi_{c}^{(i)}: \tilde{X}_{i} \rightarrow \mathbb{P}\left(\bigwedge^{n+2-c} V\right) .
$$

For $c=n+1$ we have $Z_{c}=\emptyset$ and $\left|I_{Z_{c}}\left(1+q+\ldots+q^{n+1-c}\right)\right|=\left|\mathscr{O}_{\mathbb{P}(V)}(1)\right|$, so $\varphi_{n+1}^{(i)}=\tilde{\pi}_{0} \circ \cdots \circ \tilde{\pi}_{i-1}$. For $c=2, \ldots, n+1$ we set $\varphi_{c}=\varphi_{c}^{(n)}$. Note that for $c=1$ we have $I_{Z_{c}}\left(1+q+\ldots+q^{n+1-c}\right)=\mathscr{O}_{\mathbb{P}(V)}$, so we can also define $\varphi_{1}$ as the map of $\tilde{X}$ to $\mathbb{P}\left(\bigwedge^{n+1} V\right)$.

Lemma 6.1. Let us fix $0 \leq i \leq n$. The morphism

$$
\varphi_{\tilde{X}_{i}}: \tilde{X}_{i} \rightarrow \mathbb{P}(V) \times_{k} \mathbb{P}\left(\bigwedge^{2} V\right) \times_{k} \ldots \times_{k} \mathbb{P}\left(\bigwedge^{i+1} V\right)
$$

given by $\left(\varphi_{n+1}^{(i)}, \varphi_{n}^{(i)}, \ldots, \varphi_{n+1-i}^{(i)}\right)$ is a closed embedding.

Proof. The proof is by induction on $i$, with $i=0$ being trivial. So let us assume that we know the assertion for $i-1$. By the construction and Lemma 2.3 we get a closed embedding $\tilde{X}_{i} \hookrightarrow \tilde{X}_{i-1} \times_{k} \mathbb{P}\left(\bigwedge^{i+1} V\right)$, so the lemma follows from the induction assumption.

Let us set

$$
D_{V}^{i}:=\sum_{L \in \mathscr{L}(V), \operatorname{codim} L=i} D_{L}
$$

for $i=1,2, \ldots, n$ (notation for $D_{L}$ is explained in Subsection 2.3). Let us also set $D:=\sum_{i=1}^{n} D_{V}^{i}$. The exceptional divisor of $\tilde{\pi}: \tilde{X} \rightarrow \mathbb{P}(V)$ is equal to the sum of all $D_{V}^{i}$ for $i=2, \ldots, n$. The following lemma follows by a straightforward computation:

LEMMA 6.2. Let us set

$$
H_{c}:=\frac{q^{n+2-c}-1}{q-1} H-\sum_{i=0}^{n-c} \frac{q^{i+1}-1}{q-1} D_{V}^{i+c} .
$$

Then we have $\mathscr{O}_{\tilde{X}}\left(H_{c}\right) \simeq \varphi_{c}^{*} \mathscr{O}_{\mathbb{P}\left(\wedge^{n+2-c} V\right)}(1)$ for $c=1, \ldots, n+1$. Moreover, we have

$$
K_{\tilde{X}}+\sum_{j=1}^{n} D_{V}^{j}=(q-1) \sum_{c=1}^{n} H_{c+1}
$$

Remark 6.3. 1. In cases $c=n+1$ and $c=1$ the above lemma says that $H_{n+1} \sim H$ and $H_{1} \sim 0$, so $\left(\sum_{i=0}^{n} q^{i}\right) H \sim \sum_{i=0}^{n-1}\left(\sum_{j=0}^{i} q^{j}\right) D_{V}^{i+1}$.

2. Combining the above lemma with Lemma 6.1 we get very ampleness of $K_{\tilde{X}}+\sum_{j=1}^{n} D_{V}^{j}$. Note that in [25, p. 227, Lemma] Mustafin claims that $K_{\tilde{X}}+\sum_{j=1}^{n} D_{V}^{j}$ is ample, but his proof shows only that it is strictly nef.

LEMMA 6.4. Let $\tilde{Y}$ be a smooth projective variety defined over an algebraically closed field and let $B$ be a simple normal crossing divisor. Let us set $Y=\tilde{Y}-B$ and assume that $K_{\tilde{Y}}+B$ is ample. Then any separable and dominant rational map $Y \rightarrow Y$ extends to an automorphism $\tilde{Y} \rightarrow \tilde{Y}$.

Proof. The idea of proof is the same as that of [17, Theorem 11.6], which is a general result but depending on the characteristic zero assumption and using resolution of singularities.

Let $\varphi: Y \rightarrow Y$ be a dominant separable rational map. By the valuative criterion of properness, the induced rational map $\tilde{Y} \rightarrow-\rightarrow \tilde{Y}$ is defined at every codimension one point. So there exists a closed subset $Z \subset \tilde{Y}$ of codimension at least 2 such that $\varphi$ extends to a morphism $\tilde{\varphi}: \tilde{Y}-Z \rightarrow \tilde{Y}$. The same computation as that in [17, 11.4 a] shows that $\left.\tilde{\varphi}^{*} \mathscr{O}\left(K_{\tilde{Y}}+B\right) \subset \mathscr{O}\left(K_{\tilde{Y}}+\log B\right)\right|_{\tilde{Y}-Z}$ (here we use that $\varphi$ is dominant and 
separable). Since on a normal variety sections of a locally free sheaf extend outside of codimension $2, \tilde{\varphi}$ induces injective linear maps

$$
\tilde{\varphi}_{m}^{*}: H^{0}\left(\tilde{Y}, \mathscr{O}_{\tilde{Y}}\left(m\left(K_{\tilde{Y}}+B\right)\right)\right) \rightarrow H^{0}\left(\tilde{Y}-Z, \mathscr{O}_{\tilde{Y}}\left(m\left(K_{\tilde{Y}}+B\right)\right)\right)=H^{0}\left(\tilde{Y}, \mathscr{O}_{\tilde{Y}}\left(m\left(K_{\tilde{Y}}+B\right)\right)\right) .
$$

Since the dimensions of both spaces are the same, $\tilde{\varphi}_{m}^{*}$ is an isomorphism. Now taking $m$ such that $m\left(K_{\tilde{Y}}+\right.$ $B$ ) is very ample, the required assertion follows from the commutative diagram:

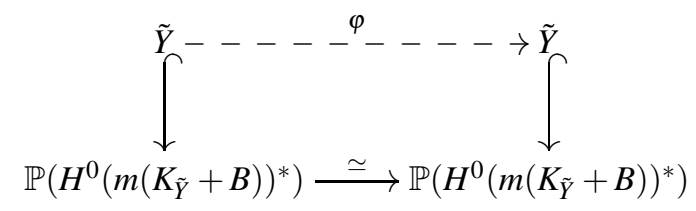

COROLlARY 6.5. Every separable and dominant k-endomorphism of $\Omega(V)$ is a k-automorphism and in particular it extends to a $k$-authomorphism of $\mathbb{P}(V)$.

Proof. By Remark 6.32 we know that $K_{\tilde{X}}+\sum_{j=1}^{n} D_{V}^{j}$ is very ample. Since $\Omega(V)=\tilde{X}-\sum_{j=1}^{n} D_{V}^{j}$, the previous lemma implies that a separable and dominant $k$-endomorphism of $\Omega(V)$ extends to an automorphism of the wonderful compactification $\tilde{X}$. So the corollary follows as in [28, p. 1222].

If $n=2$ then the wonderful compactification of $\Omega(V)$ is the blow up of $\mathbb{P}(V)$ along all $k$-rational points, so it coincides with $X_{V}$. But if $n \geq 3$ then $X_{V}$ is non-normal, so it is not isomorphic to the wonderful compactification of $\Omega(V)$.

\section{Relation to Deligne-Lusztig schemes}

The aim of this section is to provide an application of previous results to the study of Deligne-Lusztig varieties corresponding to a Coxeter element in the $A_{n}$ case. In particular, we prove that the closure of the open Deligne-Lusztig variety in the full flag variety is smooth (see Corollary 7.2 ).

Let $G$ be a connected reductive algebraic group defined over $\bar{k}$ and obtained by extension of scalars from $G_{0}$ defined over $k=\mathbb{F}_{q}$. Let us fix a $\operatorname{Fr}_{G}$-stable Borel subgroup $B \subset G$ containing a $\operatorname{Fr}_{G}$-stable maximal torus $T$. Let $X_{G}$ denote the variety of Borel subgroups of $G$. The group $G$ acts on $X_{G}$ by conjugation and there is a natural isomorphism $G / B \rightarrow X_{G}$ given by $g B \rightarrow g B g^{-1}$.

Let $P$ and $Q$ be parabolic subgroups of $G$ containing $B$. The product $G / P \times_{\bar{k}} G / Q$ with diagonal action of $G$ is a $G$-variety. This $G$-variety is $G$-equivariantly isomorphic with $G \times_{P}(G / Q)$, where the isomorphism

$$
\xi: G \times{ }_{P}(G / Q) \rightarrow G / P \times_{\bar{k}} G / Q
$$

is given by $(g, h Q) \rightarrow(g P, g h Q)$.

Let $W=N(T) / T$ be the Weyl group of $G$. For any element $w \in W$ we define the Bruhat cell $C_{w, P}:=$ $B w P \subset G / P$ and the $S c h u b e r t$ variety $S_{w, P}=\overline{C_{w, P}} \subset G / P$.

Let $\mathscr{O}(w) \subset X_{G} \times_{\bar{k}} X_{G}$ be the $G$-orbit of $(e B, \dot{w} B)$, where $\dot{w}$ is a representative of $w$ in $N(T)$. It is equal to the image of $\xi\left(G \times{ }_{B} C_{w, B}\right)$. Another way to define it is to say that $\mathscr{O}(w)$ is the preimage of $w$ under the map

$$
X_{G} \times_{\bar{k}} X_{G} \rightarrow G \backslash\left(X_{G} \times_{\bar{k}} X_{G}\right)=B \backslash G / B=W .
$$

The Zariski closure $\overline{\mathscr{O}(w)} \subset X_{G} \times_{\bar{k}} X_{G}$ is equal to the image of $\xi\left(G \times_{B} S_{w, B}\right)$. Any closed irreducible $G$ stable subset of $G / P \times_{\bar{k}} G / Q$ is the image of some $\overline{\mathscr{O}(w)}$ under the projection $X_{G} \times_{\bar{k}} X_{G} \rightarrow G / P \times_{\bar{k}} G / Q$. The image of $\overline{\mathscr{O}(w)}$ under this projection is called a $G$-Schubert variety and denoted by $S_{w, P, Q}$.

If $w=s_{1} \ldots s_{n}$ is a minimal expression for $w \in W$ then $\overline{\mathscr{O}(w)}$ has Bott-Samelson (-Demazure-Hansen) desingularization $\overline{\mathscr{O}}\left(s_{1}, \ldots, s_{n}\right) \rightarrow \overline{\mathscr{O}(w)}$. 
Let $\Gamma \subset X_{G} \times_{\bar{k}} X_{G}$ be the graph of the Frobenius endomorphism $\operatorname{Fr}_{X_{G}}$. The intersection of $\Gamma$ and $\mathscr{O}(w)$ is transversal. We denote this intersection by $X_{G}(w)$ and we call it Deligne-Lusztig scheme. This scheme is obtained by extension of scalars from a naturally defined $k$-scheme that we also denote by $X_{G}(w)$. Note that traditionally $X_{G}(w)$ is called "Deligne-Lusztig variety". This name is rather unfortunate, as usually this scheme is not a variety (often it is not irreducible). Therefore we prefer to use a slightly different name. By [6. Lemma 9.11] the graph $\Gamma$ is transverse to $\overline{\mathscr{O}}\left(s_{1}, \ldots, s_{n}\right)$ and the fibre product $\overline{X_{G}}\left(s_{1}, \ldots, s_{n}\right)$ is a smooth compactification of $X_{G}(w)$ with complement being a normal crossing divisor. We also have a canonical map

$$
\overline{X_{G}}\left(s_{1}, \ldots, s_{n}\right) \rightarrow \overline{X_{G}(w)} \subset X_{G} .
$$

Note that the closure of $X_{G}(w)$ in $X_{G}$ can be easily described using the Bruhat order in $W$ as

$$
\overline{X_{G}(w)}=\bigcup_{w^{\prime} \leq w} X_{G}\left(w^{\prime}\right) .
$$

Let $P \subset G$ be a $\operatorname{Fr}_{G}$-stable parabolic subgroup containing $B$. If we set $W_{P}=(N(T) \cap P) / T$ then to any element $\bar{w} \in W_{P} \backslash W / W_{P}$ we can associate $\mathscr{O}_{P}(\bar{w})$ defined as the preimage of $\bar{w}$ under the map

$$
G / P \times_{\bar{k}} G / P \rightarrow G \backslash\left(G / P \times_{\bar{k}} G / P\right)=P \backslash G / P=W_{P} \backslash W / W_{P} .
$$

Then we define the generalized Deligne-Lusztig scheme $X_{G, P}(\bar{w})$ as the product of the graph $\Gamma_{P} \rightarrow G / P \times_{\bar{k}}$ $G / P$ of the Frobenius endomorphism $\operatorname{Fr}_{G / P}$ and $\mathscr{O}_{P}(\bar{w}) \rightarrow G / P \times_{\bar{k}} G / P$. If $w \in W$ is some lift of $\bar{w}$, then $X_{G, P}(\bar{w})$ can be also recovered as the image of $X_{G}(w)$ under the canonical projection $G / B \rightarrow G / P$. If $\bar{w}$ is the class of $w$ we often write $X_{G, P}(w)$ instead of $X_{G, P}(\bar{w})$.

Now let $V$ be a $k$-vector space of dimension $(n+1)$ and let us consider the case when $G_{0}=\operatorname{GL}(V)$ and $G=\operatorname{GL}\left(V_{\bar{k}}\right)$. Then $W \simeq S_{n+1}$ and we consider the standard Coxeter element $w=(1,2, \ldots, n+1) \in S_{n+1}$. The corresponding Deligne-Lusztig variety $X_{G}(w)$ is the Drinfeld's half-space $\Omega(V)$. Let us fix a full flag of $k$-vector spaces

$$
V \rightarrow V_{n} \rightarrow \ldots \rightarrow V_{2} \rightarrow V_{1},
$$

where $\operatorname{dim}_{k} V_{j}=j$. Then we have a standard Borel subgroup $B_{0} \subset G_{0}$ corresponding to linear maps preserving this flag. Let us consider a sequence of parabolic groups $P_{n}=B_{0} \subsetneq P_{n-1} \subsetneq \ldots \subsetneq P_{1} \subsetneq \mathrm{GL}(V)$ so that $P_{i}$ corresponds to linear maps preserving a partial flag of the form

$$
V \rightarrow V_{i} \rightarrow \ldots \rightarrow V_{2} \rightarrow V_{1} .
$$

This induces a sequence of flag varieties

$$
\mathrm{GL}(V) / B_{0} \rightarrow \mathrm{GL}(V) / P_{n-1} \rightarrow \ldots \rightarrow \mathrm{GL}(V) / P_{1}=\mathbb{P}(V) .
$$

Note that $\operatorname{GL}(V) / P_{i} \simeq \operatorname{Flag}(V ; 1, \ldots, i)$, so for each $i$ we have the canonical embedding

$$
\operatorname{GL}(V) / P_{i} \hookrightarrow \operatorname{Grass}(V, 1) \times_{k} \operatorname{Grass}(V, 2) \times_{k} \ldots \times_{k} \operatorname{Grass}(V, i) \hookrightarrow \mathbb{P}(V) \times_{k} \mathbb{P}\left(\bigwedge^{2} V\right) \times_{k} \ldots \times_{k} \mathbb{P}\left(\bigwedge^{i} V\right),
$$

where the first map is obtained by sending an $S$-point of $\operatorname{Flag}(V ; 1, \ldots, i)$ corresponding to a partial flag

$$
V_{S} \rightarrow \mathscr{E}_{i} \rightarrow \ldots \rightarrow \mathscr{E}_{2} \rightarrow \mathscr{E}_{1}
$$

to a tuple $\left(V_{S} \rightarrow \mathscr{E}_{j}\right)_{j=1, \ldots, i}$ (cf. proof of Theorem 8.1 and the second map is the product of Plücker embeddings, given by sending the corresponding tuple to

$$
\left(V_{S} \rightarrow \operatorname{det}\left(\mathscr{E}_{1}\right), \bigwedge^{2} V_{S} \rightarrow \operatorname{det}\left(\mathscr{E}_{2}\right), \ldots, \bigwedge^{i} V_{S} \rightarrow \operatorname{det}\left(\mathscr{E}_{i}\right)\right)
$$

PROPOSITION 7.1. We have a canonical isomorphism of the sequence of closures of generalized DeligneLusztig k-schemes in flag varieties

$$
\overline{X_{G}(w)} \rightarrow \overline{X_{G, P_{n-1}}(w)} \rightarrow \ldots \rightarrow \overline{X_{G, P_{1}}(w)}=\mathbb{P}(V)
$$

with the sequence

$$
\tilde{X}=\tilde{X}_{n-1} \stackrel{\tilde{\pi}_{n-2}}{\longrightarrow} \tilde{X}_{n-2} \longrightarrow \cdots \longrightarrow \tilde{X}_{2} \stackrel{\tilde{\pi}_{1}}{\longrightarrow} \tilde{X}_{1} \stackrel{\tilde{\pi}_{0}}{\longrightarrow} \tilde{X}_{0}=\mathbb{P}(V)
$$

considered in Section 6 . 
Proof. It is well-known that $X_{G}(w) \subset \mathrm{GL}(V) / B_{0}$ is mapped isomorphically onto $\Omega(V) \subset \mathbb{P}(V)$ (see $[6$ $2.2]$ ), so the sequence of flag varieties induces isomorphisms of the corresponding generalized DeligneLusztig $k$-schemes $X_{G, P_{i}}(w)$. Since by Lemma 6.1

$$
\varphi_{\tilde{X}_{i-1}}: \tilde{X}_{i-1} \rightarrow \mathbb{P}(V) \times_{k} \mathbb{P}\left(\bigwedge^{2} V\right) \times_{k} \ldots \times_{k} \mathbb{P}\left(\bigwedge^{i} V\right)
$$

is a closed embedding and it coincides with the composition

$$
X_{G, P_{i}}(w) \subset \mathrm{GL}(V) / P_{i} \hookrightarrow \mathbb{P}(V) \times_{k} \mathbb{P}\left(\bigwedge^{2} V\right) \times_{k} \ldots \times_{k} \mathbb{P}\left(\bigwedge^{i} V\right)
$$

on the pre-image of $\Omega(V)$, we get the required assertion.

Corollary 7.2. All varieties $\overline{X_{G, P_{i}}(w)}$ are smooth. In particular, the canonical map $\overline{X_{G}}(w) \rightarrow \overline{X_{G}(w)}$ is an isomorphism.

By [11, Lemma 1] it was known that the map $\overline{X_{G}}(w) \rightarrow \overline{X_{G}(w)}$ is bijective, but it seems that the above corollary is new.

\section{Modular interpretation}

In this section we give a modular interpretation of all varieties and maps defined in Section 6 and we give the corresponding interpretation of the morphisms from Theorem 1.2

\subsection{Modular interpretation of flag varieties}

It is a standard fact that the Grassmannian is a fine moduli space for the Grassman functor of quotient modules (or vector subbundles in case of locally free sheaves). More precisely, let $\mathscr{E}$ be a coherent $\mathscr{O}_{S^{-}}$ module on a scheme $S$. A quotient module of $\mathscr{E}$ is an equivalence class of surjective maps $q: \mathscr{E} \rightarrow \mathscr{E}$ of coherent $\mathscr{O}_{S}$-modules such that two maps $q_{1}: \mathscr{E} \rightarrow \mathscr{E}_{1}^{\prime}$ and $q_{2}: \mathscr{E} \rightarrow \mathscr{E}_{2}^{\prime}$ are equivalent if their kernels give the same subsheaf of $\mathscr{E}$. Let $\underline{\operatorname{Grass}}(\mathscr{E}, r):(\mathrm{Sch} / S)^{o} \rightarrow$ Sets denote the functor associating to an $S$-scheme

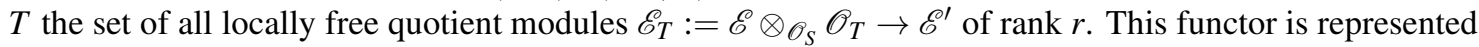
by a projective $S$-scheme denoted by $\operatorname{Grass}_{S}(\mathscr{E}, r)$ (see, e.g., [24, Lecture 5] or [16, Examples 2.2.2 and 2.2.3]). In the special case when $S=\operatorname{Spec} k$, we get a moduli interpretation of the usual Grassmanian of quotients.

The corresponding moduli interpretation of flag schemes does not seem to be well-known so we sketch it below.

Let $S$ be a scheme and let $\mathscr{E}$ be a locally free $\mathscr{O}_{S}$-module of rank $(n+1)$. Let us fix a sequence of integers $0<d_{1}<d_{2}<\cdots<d_{m}<n+1$ for some $m \geq 1$. A flag of type $\left(d_{1}, \ldots, d_{m}\right)$ in $\mathscr{E}$ is a filtration

$$
\mathscr{E}_{1} \subset \cdots \subset \mathscr{E}_{m} \subset \mathscr{E}
$$

such that the sheaf $\mathscr{E}_{i}$ is locally free of rank $d_{i}$ for every $i=1, \ldots, m$ and all quotients $\mathscr{E} / \mathscr{E}_{i}$ are locally free. If $f: T \rightarrow S$ is a morphism of schemes then we set $\mathscr{E}_{T}:=f^{*} \mathscr{E}$.

We define a functor Flag $\left(d_{1}, \ldots, d_{m} ; \mathscr{E}\right)$ from the category of $S$-schemes to the category of sets by setting

$$
\left(\underline{\operatorname{Flag}}\left(d_{1}, \ldots, d_{m} ; \mathscr{E}\right)\right)(T)=\left\{\text { flags of type }\left(d_{1}, \ldots, d_{m}\right) \text { in } \mathscr{E}_{T}\right\}
$$

For a morphism of $S$-schemes $f: T_{1} \rightarrow T_{2}$ we define the corresponding map

$$
\left.\left.\underline{(\operatorname{Flag}}\left(d_{1}, \ldots, d_{m} ; \mathscr{E}\right)\right)\left(T_{2}\right) \rightarrow \underline{(\operatorname{Flag}}\left(d_{1}, \ldots, d_{m} ; \mathscr{E}\right)\right)\left(T_{1}\right)
$$

by pull-back.

THEOREM 8.1. The functor Flag $\left(d_{1}, \ldots, d_{m} ; \mathscr{E}\right)$ is representable and the corresponding $S$-scheme Flag $\left(d_{1}, \ldots, d_{m} ; \mathscr{E}\right)$ is projective. 
Proof. Let us first recall the Grassmannian but in the setting dual to the one described above. Let $\underline{\operatorname{Grass}}(r, \mathscr{E})$ : $(\mathrm{Sch} / S)^{o} \rightarrow$ Sets denote the functor associating to an $S$-scheme $T$ the set of all locally free submodules $\mathscr{E}^{\prime} \subset \mathscr{E}_{T}$ of rank $r$ with locally free quotient $\mathscr{E}_{T} / \mathscr{E}^{\prime}$. This functor is represented by a projective $S$-scheme denoted by $\operatorname{Grass}_{S}(r, \mathscr{E})$ (here we use the fact that $\mathscr{E}$ is locally free of finite rank). This gives the required assertion for $m=1$.

In general, let us consider the functor $\underline{\operatorname{Grass}}\left(d_{1}, \mathscr{E}\right) \times_{S} \ldots \times{ }_{S} \operatorname{Grass}\left(d_{m}, \mathscr{E}\right)$ defined by associating to an $S$-scheme $T$ an $m$-tuple $\left\{\mathscr{E}_{i} \subset \mathscr{E}_{T}\right\}_{i=1, \ldots, m}$ such that $\mathscr{E}_{i}$ is locally free of rank $d_{i}$ and each quotient $\mathscr{E}_{T} / \mathscr{E}_{i}$ is locally free. The functor $\operatorname{Flag}\left(d_{1}, \ldots, d_{m} ; \mathscr{E}\right)$ is a subfunctor of the above functor defined by the conditions $\mathscr{E}_{i} \subset \mathscr{E}_{i+1}$ for $i=1, \ldots, m-1$. Note that this subfunctor is closed, i.e., for any $S$-scheme $T$ and an $m$-tuple $\left\{\mathscr{E}_{i} \subset \mathscr{E}_{T}\right\}_{i=1, \ldots, m}$ as above, there exists a closed subscheme $Z \subset T$ such that a morphism $f: T^{\prime} \rightarrow T$ of $S$-schemes factors through $Z$ if and only if the $m$-tuple $\left\{f^{*} \mathscr{E}_{i} \subset \mathscr{E}_{T^{\prime}}\right\}_{i=1, \ldots, m}$ defines a filtration, i.e., we have $f^{*} \mathscr{E}_{i} \subset f^{*} \mathscr{E}_{i+1}$ for $i=1, \ldots, m-1$. The above $Z \subset T$ can be defined by vanishing of the canonical maps $\mathscr{E}_{i} \rightarrow \mathscr{E}_{T} \rightarrow \mathscr{E}_{T} / \mathscr{E}_{i+1}$ for $i=1, \ldots, m-1$. Since the functor $\underline{\operatorname{Grass}}\left(d_{1}, \mathscr{E}\right) \times_{S} \ldots \times_{S} \underline{\operatorname{Grass}}\left(d_{m}, \mathscr{E}\right)$ is representable, the functor Flag $\left(d_{1}, \ldots, d_{m} ; \mathscr{E}\right)$ is representable by a closed subscheme of the product of Grassmannians $\operatorname{Grass}\left(d_{1}, \overline{\mathscr{E}}\right) \times_{S} \ldots \times_{S} \operatorname{Grass}\left(d_{m}, \mathscr{E}\right)$.

One can also state and prove the corresponding theorem for an arbitrary coherent $\mathscr{O}_{S}$-module $\mathscr{E}$ but then we need to use equivalence classes of quotient filtrations as in the case of Grassmannians. More precisely, let $S$ be a scheme and let $\mathscr{E}$ be a coherent $\mathscr{O}_{S}$-module. Let us fix a sequence of integers $0<d_{1}<d_{2}<\cdots<$ $d_{m}$ for some $m \geq 1$. A quotient flag of type $\left(d_{1}, \ldots, d_{m}\right)$ in $\mathscr{E}$ is a sequence of surjective maps

$$
\mathscr{E} \rightarrow \mathscr{E}_{m} \rightarrow \ldots \rightarrow \mathscr{E}_{2} \rightarrow \mathscr{E}_{1}
$$

such that the sheaf $\mathscr{E}_{i}$ is locally free of rank $d_{i}$ for every $i=1, \ldots, m$. We say that two quotient flags are equivalent if there exists a commutative diagram

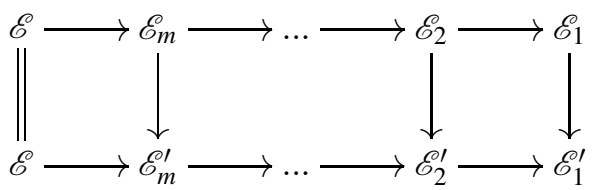

in which all vertical maps are isomorphisms. We define a functor $\underline{\operatorname{Flag}}\left(\mathscr{E} ; d_{1}, \ldots, d_{m}\right)$ from the category of $S$-schemes to the category of sets by setting

$$
\left(\underline{\operatorname{Flag}}\left(\mathscr{E} ; d_{1}, \ldots, d_{m}\right)\right)(T)=\left\{\text { equivalence classes of quotient flags of type }\left(d_{1}, \ldots, d_{m}\right) \text { in } \mathscr{E}_{T}\right\} .
$$

The functor on morphisms is again induced by pull-backs of quotient flags. Similar arguments as those used in proof of Theorem 8.1 show that $\operatorname{Flag}\left(\mathscr{E} ; d_{1}, \ldots, d_{m}\right)$ is represented by a projective $S$-scheme $\operatorname{Flag}\left(\mathscr{E} ; d_{1}, \ldots, d_{m}\right) / S$. If $\mathscr{E}$ is locally free of rank $(n+1)$ then the $S$-scheme $\operatorname{Flag}\left(\mathscr{E} ; d_{1}, \ldots, d_{m}\right) / S$ is isomorphic to the $S$-scheme Flag $\left(n+1-d_{m}, \ldots, n+1-d_{1} ; \mathscr{E}\right) / S$. The isomorphism is realised by sending a quotient flag $\mathscr{E} \rightarrow \mathscr{E}_{m} \rightarrow$ $\ldots \rightarrow \mathscr{E}_{2} \rightarrow \mathscr{E}_{1}$ to $\operatorname{ker}\left(\mathscr{E} \rightarrow \mathscr{E}_{1}\right) \subset \ldots \subset \operatorname{ker}\left(\mathscr{E} \rightarrow \mathscr{E}_{m}\right) \subset \mathscr{E}$

\subsection{Moduli spaces of F-flags}

Note that in notation of Section $7 X_{\mathrm{GL}(V)}=\operatorname{Flag}(V ; 1, \ldots, n)=\operatorname{Flag}\left(1, \ldots, n ; V^{*}\right)$ and the definition of flag schemes does not require a choice of a flag. However, Shubert varieties are defined using choice of a Borel subgroup, i.e., a choice of the full flag in $V$. Nevertheless, it is possible to use the Frobenius morphism to give a modular interpretation of the above closures of our generalized Deligne-Lusztig $k$-schemes without any choice of a flag in $V$. In fact, we give a more general construction that is useful also in other contexts.

Let $k=\mathbb{F}_{q}$ and let $V$ be a $k$-vector space of dimension $(n+1)$. Let us fix a sequence of integers $0<d_{1}<d_{2}<\cdots<d_{m}<n+1$ for some $m \geq 1$. Let us also fix a subset $S \subset\left\{d_{1}, \ldots, d_{m}\right\} \times\left\{d_{1}, \ldots, d_{m}\right\}$. For $s \in S$ we denote by $s_{1}$ the first coordinate and by $s_{2}$ the second one. Note that if $T$ is a $k$-scheme and $\mathscr{E}_{1} \subset \cdots \subset \mathscr{E}_{m} \subset V_{T}$ is a flag of type $\left(d_{1}, \ldots, d_{m}\right)$ then $\operatorname{Fr}^{*} \mathscr{E}_{1} \subset \cdots \subset \mathrm{Fr}^{*} \mathscr{E}_{m} \subset \mathrm{Fr}^{*} V_{T}=V_{T}$ is also a flag of type $\left(d_{1}, \ldots, d_{m}\right)$ in $V_{T}$. We say that a flag $\mathscr{E}_{1} \subset \cdots \subset \mathscr{E}_{m} \subset V_{T}$ of type $\left(d_{1}, \ldots, d_{m}\right)$ is an $F$-flag of type $S$ if for all $s \in S$ we have $\operatorname{Fr}^{*} \mathscr{E}_{s_{1}} \subset \mathscr{E}_{s_{2}}$ if $s_{1} \leq s_{2}$ and $\mathscr{E}_{s_{2}} \subset \operatorname{Fr}^{*} \mathscr{E}_{s_{1}}$ if $s_{1}>s_{2}$. 
We define a functor $\underline{\text { Flag }}^{F}(S ; V)$ from the category of $k$-schemes to the category of sets by setting

$$
\left.\underline{\operatorname{Flag}}^{F}(S ; V)\right)(T)=\left\{\text { F-flags } \mathscr{E}_{1} \subset \cdots \subset \mathscr{E}_{m} \subset V_{T} \text { of type } S\right\}
$$

For a morphism of $S$-schemes $f: T_{1} \rightarrow T_{2}$ the corresponding map

$$
\left.\left.\underline{\operatorname{Flag}}^{F}(S ; V)\right)\left(T_{2}\right) \rightarrow \underline{\operatorname{Flag}}^{F}(S ; V)\right)\left(T_{1}\right)
$$

is defined by pull-back.

Similarly, one can define functors for quotient flags.

Since the conditions defining the functor $\underline{\operatorname{Flag}}^{F}(S ; V)$ are closed, in the same way as Theorem 8.1 one can prove the following theorem:

THEOREM 8.2. The functor Flag $^{F}(S ; V)$ is representable and the corresponding $k$-scheme is a projective subscheme of $\operatorname{Flag}\left(d_{1}, \ldots, d_{m} ; \overline{V)}\right.$.

If $S=\left\{\left(s_{1}, s_{2}\right) \in\left\{d_{1}, \ldots, d_{m}\right\} \times\left\{d_{1}, \ldots, d_{m}\right\}: s_{1}<s_{2}\right\}$ then the scheme $\operatorname{Flag}^{F}(S ; V)$ is denoted by $\operatorname{Flag}^{F}\left(d_{1}, \ldots, d_{m} ; V\right)$. If $S=\left\{\left(s_{1}, s_{2}\right) \in\left\{d_{1}, \ldots, d_{m}\right\} \times\left\{d_{1}, \ldots, d_{m}\right\}: s_{1}>s_{2}\right\}$ then the scheme Flag ${ }^{F}(S ; V)$ is denoted by $\operatorname{Flag}_{F}\left(d_{1}, \ldots, d_{m} ; V\right)$.

Proposition 8.3. We have $\operatorname{Flag}^{F}(1, \ldots, m ; V) \simeq \tilde{X}_{m-1}$ for $m=1, \ldots, n$.

Proof. Restricting the universal family of flags on $X_{\mathrm{GL}(V)}$ to $\tilde{X}_{m-1}$ and using the universal property of $\operatorname{Flag}^{F}(1, \ldots, m ; V)$ we get a canonical map $\tilde{X}_{m-1} \rightarrow \operatorname{Flag}^{F}(1, \ldots, m ; V)$. This map is compatible with embeddings into $X_{\mathrm{GL}(V)}$ and bijective on $\bar{k}$-points, so it is an isomorphism.

Note that there are many interesting, natural maps between moduli spaces of F-flags. They are given by Frobenius pull-backs of some factors in the flag. For example if $m=2$ then we have the map

$$
\operatorname{Flag}^{F}\left(d_{1}, d_{2} ; V\right) \rightarrow \operatorname{Flag}_{F}\left(d_{1}, d_{2} ; V\right)
$$

given on functors by sending flag $\mathscr{E}_{1} \subset \mathscr{E}_{2} \subset V_{T}$ to $\mathrm{Fr}^{*} \mathscr{E}_{1} \subset \mathscr{E}_{2} \subset V_{T}$ and the map

$$
\operatorname{Flag}_{F}\left(d_{1}, d_{2} ; V\right) \rightarrow \operatorname{Flag}^{F}\left(d_{1}, d_{2} ; V\right)
$$

given by sending flag $\mathscr{E}_{1} \subset \mathscr{E}_{2} \subset V_{T}$ to $\mathscr{E}_{1} \subset \mathrm{Fr}^{*} \mathscr{E}_{2} \subset V_{T}$. The composition of these maps sends $\mathscr{E}_{1} \subset \mathscr{E}_{2} \subset V_{T}$ to $\operatorname{Fr}^{*} \mathscr{E}_{1} \subset \operatorname{Fr}^{*} \mathscr{E}_{2} \subset V_{T}$, so it is equal to the Frobenius endomorphism of $\operatorname{Flag}^{F}\left(d_{1}, d_{2} ; V\right)$.

Let us take $n=2, d_{1}=1$ and $d_{2}=2$. Then the above maps correspond to $\varphi_{X_{V}}$ and $\varphi_{X_{V^{*}}}$. We have a canonical isomorphism $\operatorname{Flag}^{F}(1,2 ; V) \simeq \operatorname{Flag}_{F}\left(1,2 ; V^{*}\right)$ given by sending flag $\mathscr{E}_{1} \subset \mathscr{E}_{2} \subset V_{T}$ to $\mathscr{E}_{1}^{\prime}=$ $\operatorname{ker}\left(V_{T}^{*} \rightarrow \mathscr{E}_{2}^{*}\right) \subset \mathscr{E}_{2}^{\prime}=\operatorname{ker}\left(V_{T}^{*} \rightarrow \mathscr{E}_{1}^{*}\right) \subset V_{T}^{*}$. Therefore Flag ${ }^{F}(1,2 ; V)$ is non-canonically isomorphic to $\operatorname{Flag}_{F}(1,2 ; V)$. If we choose an isomorphism $V \simeq V^{*}$ then the above maps become the same endomorphism of Flag ${ }^{F}(1,2 ; V)$. One can also obtain a similar modular interpretation of the maps from Theorem 5.6 in higher dimensions.

\section{Logarithmic tangent bundle}

Let $X$ be a smooth projective variety with a divisor $D$, both defined over some field $k$. Let $j: U \hookrightarrow X$ be the open subset where the pair $(X, D)$ is smooth. Then we can consider the logarithmic tangent bundle $T_{U}\left(-\left.\log D\right|_{U}\right)$. Since $U$ is a big open subset of $X$ (i.e., the codimension of the complement is at least 2), the sheaf $T_{X}(-\log D):=j_{*}\left(T_{U}\left(-\left.\log D\right|_{U}\right)\right)$ is reflexive and it is called the logarithmic tangent sheaf of $(X, D)$. We will also use a well-known interpretation of $T_{X}(-\log D)$ as the sheaf of derivations preserving the ideal sheaf of $D$.

We say that $D$ is free if $T_{X}(-\log D)$ is locally free. 
As in Subsection 2.6 we consider the maps $\theta_{i}: \mathscr{O}_{\mathbb{P}(V)}\left(-q^{i}+1\right) \rightarrow T_{\mathbb{P}(V)}$ defined by $\left(\mathrm{Fr}^{i}\right)^{*} s \otimes \mathrm{id}_{\mathscr{O}_{\mathbb{P}(V)}(1)}$ for $i=1, \ldots, n$. Let us set

$$
\theta=\left(\theta_{1}, \ldots, \theta_{n}\right): \bigoplus_{i=1}^{n} \mathscr{O}_{\mathbb{P}(V)}\left(-q^{i}+1\right) \rightarrow T_{\mathbb{P}(V)} .
$$

By Lemma 2.1 this map is an isomorphism outside of the sum $B$ of all $k$-linear hyperplanes in $\mathbb{P}(V)$. In particlar, $\theta$ is injective as a map of $\mathscr{O}_{\mathbb{P}(V)}$-modules.

PROPOSITION 9.1. $\theta$ induces an isomorphism $T_{\mathbb{P}(V)}(-\log B) \simeq \bigoplus_{i=1}^{n} \mathscr{O}_{\mathbb{P}(V)}\left(-q^{i}+1\right)$. In particular, $B$ is a free hyperplane arrangement on $\mathbb{P}(V)$.

Proof. Let $\bar{B}$ be the sum of all $k$-linear hyperplanes in vector space $V^{*}$ and let $v: V_{0}^{*}:=V^{*}-\{0\} \rightarrow \mathbb{P}(V)$ denote the canonical projection. Let us fix linear coordinates in $V^{*}$ and let us consider derivations $\delta_{i}:=$ $\sum_{j=0}^{n} x_{j}^{q^{i}} \frac{\partial}{\partial x_{j}}$ of $\mathscr{O}_{V^{*}}$. If $a_{j} \in \mathbb{F}_{q}$ then

$$
\delta_{i}\left(\sum a_{j} x_{j}\right)=\left(\sum a_{j} x_{j}\right)^{q^{i}} \in\left(\sum a_{j} x_{j}\right) \mathscr{O}_{V^{*}}
$$

so derivation $\delta_{i}$ preserves the ideal of $\bar{B}$. Hence the image of $\left(\delta_{0}, \ldots, \delta_{n}\right): \mathscr{O}_{V^{*}}^{n+1} \rightarrow T_{V^{*}}$ lies in $T_{V^{*}}(-\log \bar{B})$. Then comparison of the first Chern classes shows that the image of this map coincides with $T_{V^{*}}(-\log \bar{B})$ (this fact is usually called Saito's criterion; see also [26, Example 4.24]). Euler's exact sequence implies that we have an exact sequence

$$
\left.0 \rightarrow \mathscr{O}_{V_{0}^{*}} \stackrel{\delta_{0}}{\longrightarrow} T_{V^{*}}(-\log \bar{B})\right|_{V_{0}^{*}} \stackrel{\eta}{\longrightarrow} v^{*} T_{\mathbb{P}(V)}(-\log B) .
$$

Since $v$ is a $\mathbb{G}_{m}$-torsor and $T_{\mathbb{P}(V)}(-\log B)$ is reflexive, $v^{*} T_{\mathbb{P}(V)}(-\log B)$ is also reflexive. On the other hand, $\delta_{0}$ defines a nowhere vanishing section of $\left.T_{V^{*}}(-\log \bar{B})\right|_{V_{0}^{*}}$, so the cokernel of the corresponding map is locally free. It follows that $\eta$ induces an injection of a locally free sheaf into a reflexive sheaf. Since $\eta$ is generically surjective, it is surjective and $v^{*} T_{\mathbb{P}(V)}(-\log B)$ is locally free. Hence using descent we see that $T_{\mathbb{P}(V)}(-\log B)$ is also locally free. Since the maps $\eta \delta_{i}: \mathscr{O}_{V_{0}^{*}} \rightarrow v^{*} T_{\mathbb{P}(V)}(-\log B)$ for $i=1, \ldots, n$ descend to $\theta_{i}: \mathscr{O}_{\mathbb{P}(V)}\left(-q^{i}+1\right) \rightarrow T_{\mathbb{P}(V)}(-\log B)$, we get the required assertion.

\section{1 -forms and foliations on the wonderful compactification}

For $j=1, \ldots, n$ let us denote by $\mathscr{F}_{j} \subset T_{\mathbb{P}(V)}$ the image of $\bigoplus_{i=1}^{j} \mathscr{O}_{\mathbb{P}(V)}\left(-q^{i}+1\right)$ under $\left(\theta_{1}, \ldots, \theta_{j}\right)$. By Corollary 3.2 the map $V \otimes \mathscr{O}_{\mathbb{P}(V)} \rightarrow \bigoplus_{i=0}^{j} \mathscr{O}_{\mathbb{P}(V)}\left(q^{i}\right)$ is surjective outside of $Z_{n+1-j}$. By Euler's exact sequence this map induces a generically surjective map

$$
\Omega_{\mathbb{P}(V)} \rightarrow \bigoplus_{i=1}^{j} \mathscr{O}_{\mathbb{P}(V)}\left(q^{i}-1\right)
$$

Dualizing this map we see that for $j=1, \ldots, n-1$ the quotient $T_{\mathbb{P}(V)} / \mathscr{F}_{j}$ is torsion free and locally free outside of $Z_{n+1-j}$.

An easy computation shows that

$$
\left[\theta_{i}, \theta_{j}\right]=\theta_{j}-\theta_{i}
$$

so $\left[\mathscr{F}_{j}, \mathscr{F}_{j}\right] \subset \mathscr{F}_{j}$. Using this equality, Lemma 2.8 and Jacobson's identity one can also check that $\mathscr{F}_{j}^{p} \subset$ $\mathscr{F}_{j}$, so $\mathscr{F}_{j} \subset T_{\mathbb{P}(V)}$ is a 1 -foliation for $j=1, \ldots, n-1$. By Proposition 9.1 we have an increasing filtration

$$
\mathscr{F}_{1} \subset \mathscr{F}_{2} \subset \ldots \subset \mathscr{F}_{n}=T_{\mathbb{P}(V)}(-\log B) \subset T_{\mathbb{P}(V)} .
$$

Note that $T_{\mathbb{P}(V)}(-\log B) / \mathscr{F}_{j}$ is locally free, so $\mathscr{F}_{j} \subset T_{\mathbb{P}(V)}(-\log B)$ are "smooth logarithmic 1-foliations" (note however that the pair $(\mathbb{P}(V), B)$ is not $\log$ smooth). One can also show that singularities of $\mathscr{F}_{j} \subset T_{\mathbb{P}(V)}$ are resolved by passing to $\tilde{X}_{j}$, i.e., the foliation induced by $\mathscr{F}_{j} \subset T_{\mathbb{P}(V)}$ on $T_{\tilde{X}_{j}}$ is smooth. Hirokado's 
construction from [13] uses this fact in the special case when $n=3$ and $j=1$. Since we do not need this fact in general, we skip its proof.

Let us consider the maximal 1-foliation $\mathscr{F}_{n-1} \subset T_{\mathbb{P}(V)}$. We have an exact sequence

$$
0 \rightarrow \mathscr{F}_{n-1} \rightarrow T_{\mathbb{P}(V)} \rightarrow I_{Z}\left(\left(1+q+\ldots+q^{n-1}\right)+1\right) \rightarrow 0
$$

Let $\omega$ be a rational 1 -form corresponding to the last non-zero map in this sequence. It fits into an exact sequence

$$
0 \rightarrow \mathscr{O}_{\mathbb{P}(V)}\left(-1-q-\ldots-q^{n-1}\right) \stackrel{\omega}{\longrightarrow} \Omega_{\mathbb{P}(V)}(1) \stackrel{\theta(-1)^{\vee}}{\longrightarrow} \bigoplus_{i=1}^{n-1} \mathscr{O}_{\mathbb{P}(V)}\left(q^{i}\right) .
$$

Let us fix a basis $e_{0}, \ldots, e_{n}$ of $V$. Then we have

$$
\omega=\sum_{i=0}^{n}(-1)^{i} \Delta_{q}\left(x_{0}, \ldots, \hat{x}_{i}, \ldots, x_{n}\right) e_{i},
$$

when treated as an element of

$$
H^{0}\left(\mathscr{O}_{\mathbb{P}(V)}\left(1+q+\ldots+q^{n-1}\right) \otimes \Omega_{\mathbb{P}(V)}(1)\right) \subset H^{0}\left(\mathscr{O}_{\mathbb{P}(V)}\left(1+q+\ldots+q^{n-1}\right) \otimes V\right) .
$$

PROPOSITION 10.1. The rational 1-form $\omega$ induces a 1-foliation on the wonderful compactification $\tilde{X}$ of Drinfeld's half-space $\Omega(V)$. More precisely, we have a short exact sequence

$$
0 \rightarrow \tilde{\mathscr{F}}_{n-1} \rightarrow T_{\tilde{X}} \rightarrow \mathscr{O}_{\tilde{X}}\left(\left(\frac{q^{n}+q-2}{q-1}\right) H-\sum_{i=1}^{n-1} \frac{q^{i}+q-2}{q-1} D_{V}^{i+1}\right) \rightarrow 0
$$

in which $\tilde{\mathscr{F}}_{n-1}$ is a smooth 1-foliation of rank $(n-1)$ induced by $\mathscr{F}_{n-1} \subset T_{\mathbb{P}(V)}$.

Proof. Let $U=\operatorname{Spec} k\left[t_{1}, \ldots, t_{n}\right] \subset \mathbb{P}(V)$ correspond to $D_{+}\left(x_{0}\right)$ with $t_{i}=x_{i} / x_{0}$. Then

$$
\left.\omega\right|_{U}=\sum_{i=1}^{n}(-1)^{i} \Delta_{q}\left(1, t_{1}, \ldots, \hat{t}_{i}, \ldots, t_{n}\right) d t_{i}
$$

Let us note that

$$
\begin{aligned}
h_{n} & :=\prod_{i=1}^{n} \prod_{\left(a_{i}, \ldots, a_{n}\right) \in k^{n+1-i}}\left(1+a_{i} s_{i}+a_{i+1} s_{i} s_{i+1}+\ldots+a_{n} s_{i} \ldots s_{n}\right)=\prod_{i=1}^{n} \frac{\Delta_{q}\left(1, s_{i}, s_{i} s_{i+1}, \ldots, s_{i} \ldots s_{n}\right)}{\Delta_{q}\left(s_{i}, s_{i} s_{i+1}, \ldots, s_{i} \ldots s_{n}\right)} \\
& =\frac{\Delta_{q}\left(1, s_{1}, s_{1} s_{2}, \ldots, s_{1} \ldots s_{n}\right)}{\Delta_{q}\left(s_{n}\right)} \cdot \prod_{i=1}^{n-1} \frac{\Delta_{q}\left(1, s_{i+1}, s_{i+1} s_{i+2}, \ldots, s_{i+1} \ldots s_{n}\right)}{\Delta_{q}\left(s_{i}, s_{i} s_{i+1}, \ldots, s_{i} \ldots s_{n}\right)}=\frac{\Delta_{q}\left(1, s_{1}, s_{1} s_{2}, \ldots, s_{1} \ldots s_{n}\right)}{\prod_{i=1}^{n} s_{i}^{q^{0}+q^{1}+\ldots+q^{n-i}}} .
\end{aligned}
$$

The map $\pi: \tilde{X} \rightarrow \mathbb{P}(V)$ is covered by charts that are given

$$
\pi: \tilde{U}:=D\left(h_{n}\right) \subset \operatorname{Spec} k\left[s_{1}, \ldots, s_{n}\right] \rightarrow \operatorname{Spec} k\left[t_{1}, \ldots, t_{n}\right]
$$

with $\pi^{*}\left(t_{i}\right)=\prod_{j \leq i} s_{j}$ (see [28, proof of Lemma 1.3] but note that the last diagram on [ibid., p. 1214] needs to be restricted to an open subset of $Y$ as the map $q$ is given by a different formula on whole Spec $k\left[t_{1}, \ldots, t_{n}\right]$; there is also a misprint in description of the exceptional divisor in [ibid.]). The exceptional divisor of this map is given by $\operatorname{div}\left(s_{1} \ldots s_{n-1}\right)$ and we have

$$
\pi^{*}\left(\frac{d t_{i}}{t_{i}}\right)=\sum_{j \leq i} \frac{d s_{j}}{s_{j}}
$$


Therefore we obtain

$$
\begin{aligned}
\left.\pi^{*} \omega\right|_{\tilde{U}} & =\sum_{i=1}^{n}(-1)^{i} \Delta_{q}\left(1, s_{1}, \ldots, \widehat{s_{1} \ldots s_{i}}, \ldots, s_{1} \ldots s_{n}\right)\left(s_{1} \ldots s_{i} \sum_{j \leq i} \frac{d s_{j}}{s_{j}}\right)= \\
& =\sum_{j=1}^{n}\left(\sum_{i \geq j}(-1)^{i} s_{1} \ldots \hat{s}_{j} \ldots s_{i} \cdot \Delta_{q}\left(1, s_{1}, \ldots, \widehat{s_{1} \ldots s_{i}}, \ldots, s_{1} \ldots s_{n}\right)\right) d s_{j} .
\end{aligned}
$$

The coefficient of $\left.\pi^{*} \omega\right|_{\tilde{U}}$ at $d s_{n}$ is equal to

$$
(-1)^{n} s_{1} \ldots s_{n-1} \Delta_{q}\left(1, s_{1}, \ldots, s_{1} \ldots s_{n-1}\right)=(-1)^{n} h_{n-1} \cdot \prod_{i=1}^{n-1} s_{i}^{\left(q^{0}+q^{1}+\ldots+q^{(n-1)-i}\right)+1} .
$$

It is easy to see that the coefficients of $\left.\pi^{*} \omega\right|_{\tilde{U}}$ at $d s_{j}$ for $j=1, \ldots, n-1$ are also divisible by $\prod_{i=1}^{n-1} s_{i}^{\left(q^{0}+q^{1}+\ldots+q^{(n-1)-i}\right)+1}$. Since the polynomial $h_{n-1}$ divides $h_{n}$, we can write

$$
\left.\pi^{*} \omega\right|_{\tilde{U}}=\prod_{i=1}^{n-1} s_{i}^{\left(q^{0}+q^{1}+\ldots+q^{(n-1)-i}\right)+1}\left(\alpha_{1} d s_{1}+\ldots+\alpha_{n} d s_{n}\right)
$$

for some $\alpha_{i} \in k\left[s_{1}, \ldots, s_{n}\right]$ such that $\alpha_{n}$ is invertible in $\mathscr{O}\left(D\left(h_{n}\right)\right)=k\left[s_{1}, \ldots, s_{n}\right]_{h_{n}}$. This shows that $\pi^{*} \omega$ defines the map

$$
\mathscr{O}_{\tilde{X}}\left(-\left(\frac{q^{n}+q-2}{q-1}\right) H+\sum_{i=1}^{n-1} \frac{q^{i}+q-2}{q-1} D_{V}^{i+1}\right) \rightarrow \Omega_{\tilde{X}}
$$

with locally free cokernel. After dualizing we get the required smooth 1-foliation on the wonderful compactification of $\Omega(V)$.

A simple computation of the canonical divisor of a blow up shows that

$$
K_{\tilde{X}}=-(n+1) H+\sum_{i=1}^{n-1} i D_{V}^{i+1}
$$

Therefore we have

$$
\begin{aligned}
c_{1}\left(\operatorname{det} \tilde{\mathscr{F}}_{n-1}\right) & =-K_{\tilde{X}}-\left(\left(\frac{q^{n}-1}{q-1}+1\right) H-\sum_{i=1}^{n-1}\left(\frac{q^{i}-1}{q-1}+1\right) D_{V}^{i+1}\right) \\
& =-\left(\frac{q^{n}-1}{q-1}-n\right) H+\sum_{i=1}^{n-1}\left(\frac{q^{i}-1}{q-1}-i+1\right) D_{V}^{i+1} .
\end{aligned}
$$

Let us note that

$$
\pi^{*}\left(t_{i} \frac{\partial}{\partial t_{i}}\right)=\left\{\begin{array}{cc}
s_{i} \frac{\partial}{\partial s_{i}}-s_{i+1} \frac{\partial}{\partial s_{i+1}} & \text { for } i<n, \\
s_{n} \frac{\partial}{\partial s_{n}} & \text { for } i=n .
\end{array}\right.
$$

Let $\delta_{j}$ be the rational vector field corresponding to $\theta_{j}$. Then in the notation of proof of Proposition 10.1 we can write

$$
\left.\delta_{j}\right|_{U}=\sum_{i=1}^{n}\left(t_{i}^{q^{j}-1}-1\right) t_{i} \frac{\partial}{\partial t_{i}}
$$

Therefore after a short computation we get

$$
\left.\pi^{*} \delta_{j}\right|_{\tilde{U}}=\left(s_{1}^{q^{j}-1}-1\right) s_{1} \frac{\partial}{\partial s_{1}}+s_{1}^{q^{j}-1}\left(s_{2}^{q^{j}-1}-1\right) s_{2} \frac{\partial}{\partial s_{2}}+\ldots+\left(s_{1} \ldots s_{n-1}\right)^{q^{j}-1}\left(s_{n}^{q^{j}-1}-1\right) s_{n} \frac{\partial}{\partial s_{n}} .
$$

Let us set $D:=\sum_{i=1}^{n} D_{V}^{i}$. 
PROPOSITION 10.2. The rational vector field $\delta_{1}$ induces a smooth 1 -foliation

$$
\tilde{\mathscr{F}}_{1} \simeq \mathscr{O}_{\tilde{X}}\left(-(q-1) H+D_{V}^{n}\right) \subset T_{\tilde{X}}
$$

on the wonderful compactification $\tilde{X}$ of Drinfeld's half-space $\Omega(V)$.

Proof. Taking $j=1$ in the above formula on $\left.\pi^{*} \delta_{j}\right|_{\tilde{U}}$, we see that $\left.\pi^{*} \delta_{1}\right|_{\tilde{U}}$ vanishes with order one along $\operatorname{div}\left(s_{1}\right)$ and it does not vanish along any other component of the exceptional divisor $\operatorname{div}\left(s_{1} \ldots s_{n-1}\right)$. So the corresponding foliation $\tilde{\mathscr{F}}_{1}$ is isomorphic to $\mathscr{O}_{\tilde{X}}\left(-(q-1) H+D_{V}^{n}\right)$. Since $\mathscr{F}_{1} \subset T_{\mathbb{P}(V)}$ has singularities only along $Z_{n}=\mathbb{P}(V)\left(\mathbb{F}_{q}\right)$, this foliation can have singular points only on $\operatorname{div}\left(s_{1}\right)$. Since $s_{1}^{q-1}-1$ does not vanish on this divisor, $\mathscr{F}_{1}$ is smooth.

The above proposition implies that although $\mathscr{F}_{j} \subset T_{\mathbb{P}(V)}(-\log B)$ for all $j$, already $\tilde{\mathscr{F}}_{1}$ is not contained in $T_{\tilde{X}}(-\log D)$. One can generalize the above proposition and describe the filtration

$$
\tilde{\mathscr{F}}_{1} \subset \tilde{\mathscr{F}}_{2} \subset \ldots \subset \tilde{\mathscr{F}}_{n}=T_{\tilde{X}}
$$

and the induced filtration on $T_{\tilde{X}}(-\log D)$. More precisely, each $\tilde{\mathscr{F}}_{j}$ is a smooth foliation of rank $i$ and

$$
\tilde{\mathscr{F}}_{j} / \tilde{\mathscr{F}}_{j-1} \simeq \mathscr{O}_{\tilde{X}}\left(-(q-1) H_{n+2-j}+D_{V}^{n+1-j}\right)
$$

(see Proposition 12.1 for the 3-dimensional case).

Let $\mathscr{L}_{j}$ be the image of $\theta_{j}$ in $T_{\mathbb{P}(V)}$ and let $\tilde{\mathscr{L}}_{j} \subset T_{\tilde{X}}$ be the corresponding foliation. Let us also set $\mathscr{M}_{j}:=\tilde{\mathscr{L}}_{j} \cap T_{\tilde{X}}(-\log D)$. Note that for $j \geq 2$ the foliation $\tilde{\mathscr{L}}_{j}$ is not smooth as $\pi: \tilde{X} \rightarrow \mathbb{P}(V)$ is an isomorphism over $\mathbb{P}(V)-Z$ and for $j \geq 2$ the foliation $\mathscr{L}_{j} \subset T_{\mathbb{P}(V)}$ has singularities along a non-empty set of $\mathbb{F}_{q^{j}}$-points of $\mathbb{P}(V)-Z$. However, $\mathscr{M}_{j} \simeq \mathscr{O}_{\tilde{X}}\left(-\left(q^{j}-1\right) H\right)$ and $T_{\tilde{X}}(-\log D) / \mathscr{M}_{j}$ is locally free. In particular, we have

$$
\bigoplus_{i=1}^{n} \mathscr{M}_{i} \simeq \bigoplus_{i=1}^{n} \mathscr{O}_{\tilde{X}}\left(-\left(q^{i}-1\right) H\right) \subset T_{\tilde{X}}(-\log D)
$$

Let us set $\mathscr{G}_{0}=0$ and $\mathscr{G}_{j}=\tilde{\mathscr{F}}_{j} \cap T_{\tilde{X}}(-\log D)$ for $j=1, \ldots, n$. By definition $\mathscr{G}_{j}$ is the saturation of $\bigoplus_{i=1}^{j} \mathscr{M}_{i}$ in $T_{\tilde{X}}(-\log D)$. Then the quotients of the filtration $\mathscr{G}_{\bullet}$ of $T_{\tilde{X}}(-\log D)$ are given by

$$
\mathscr{G}_{j} / \mathscr{G}_{j-1} \simeq \mathscr{O}_{\tilde{X}}\left(-(q-1) H_{n+2-j}\right)=\mathscr{O}_{\tilde{X}}\left(-\left(q^{j}-1\right) H+\sum_{i=0}^{j-2}\left(q^{i+j-1}-1\right) D_{V}^{n-i}\right)
$$

for $j=1, \ldots, n$. We leave the proofs of these facts to an interested reader (for the last fact see also Proposition 11.1 in the surface case).

\section{Rational surfaces whose cotangent bundle contains an ample line bundle}

Let $\pi: X \rightarrow \mathbb{P}^{2}$ be the blow up of $\mathbb{P}^{2}$ along $Z=\mathbb{P}^{2}\left(\mathbb{F}_{q}\right)$. We denote by $H$ the pull back of the hyperplane divisor on $\mathbb{P}^{2}$ and by $E=\sum E_{i}$ the exceptional divisor of $\pi$. Let $\tilde{L}_{i}$ for $i=1, \ldots, q^{2}+q+1$ be the strict transforms of lines $L_{i}$ passing through at least two points of $Z$. Let us set $B=\sum L_{i}, \tilde{B}=\sum \tilde{L}_{i}$ and $D=\tilde{B}+E$.

The following proposition should be compared with its non-explicit version [20, Lemma 8.3]:

PROPOSITION 11.1. We have short exact sequences

$$
\begin{gathered}
0 \rightarrow \mathscr{O}_{X}(-(q+2) H+2 E) \rightarrow \Omega_{X} \rightarrow \mathscr{O}_{X}((q-1) H-E) \rightarrow 0, \\
0 \rightarrow \mathscr{O}_{X}\left(\left(q^{2}-1\right) H-(q-1) E\right) \rightarrow \Omega_{X}(\log \tilde{B}) \rightarrow \mathscr{O}_{X}((q-1) H-E) \rightarrow 0
\end{gathered}
$$

and

$$
0 \rightarrow \mathscr{O}_{X}\left(\left(q^{2}-1\right) H-(q-1) E\right) \rightarrow \Omega_{X}(\log D) \rightarrow \mathscr{O}_{X}((q-1) H) \rightarrow 0 .
$$

In particular, $\Omega_{\tilde{X}}(\log \tilde{B})$ and $\Omega_{\tilde{X}}(\log D)$ contain $M=\left(q^{2}-1\right) H-(q-1) E$ such that $M^{2}>0$ and $M H>0$. 
Proof. The first sequence comes from dualizing the inclusion $\tilde{\mathscr{F}}_{1} \subset T_{X}$ described in Proposition 10.1. In the notation of proof of this proposition we have

$$
\left.\pi^{*} \delta_{1}\right|_{\tilde{U}}=s_{1}\left[\left(s_{1}^{q-1}-1\right) \frac{\partial}{\partial s_{1}}+s_{1}^{q-2}\left(s_{2}^{q-1}-1\right) s_{2} \frac{\partial}{\partial s_{2}}\right] .
$$

Note that $\delta_{1}$ defines $\mathscr{O}_{\mathbb{P}^{2}}(1-q) \rightarrow T_{\mathbb{P}^{2}}(-\log B)$ and the cokernel is locally free (see Proposition 9.1). Since $\pi^{*} \delta_{1}$ vanishes with order 1 along the exceptional divisor $E$, it defines a map $\mathscr{O}_{X}(-(q-1) H+E) \rightarrow$ $T_{X}(-\log \tilde{B})$, which by the above is locally free outside of $E$. It is also locally free outside of $\tilde{B}$, so we need only to check that the cokernel is locally free at all points of intersection of $\tilde{B}$ and $E$. On $\tilde{U}$ the intersection $\tilde{B} \cap \pi^{-1}(0)$ is just 1 point given by $s_{1}=s_{2}=0$. Since at this point $T_{X}(-\log \tilde{B})$ has local generators $\frac{\partial}{\partial s_{1}}$ and $s_{2} \frac{\partial}{\partial s_{2}}$, the cokernel of $\mathscr{O}_{X}(-(q-1) H+E) \rightarrow T_{X}(-\log \tilde{B})$ is locally free also at this point. After dualizing this shows existence of the second exact sequence.

To see the last exact sequence note that by similar arguments $\pi^{*} \delta_{1}$ defines also the map $\mathscr{O}_{X}(-(q-$ 1) $H) \rightarrow T_{X}(-\log D)$ with locally free cokernel.

It is interesting to note that the last sequence from the above proposition is usually (but not always) split. It will be more convenient to consider the dual sequence.

PROPOSITION 11.2. The short exact sequence

$$
0 \rightarrow \mathscr{O}_{X}(-(q-1) H) \rightarrow T_{X}(-\log D) \rightarrow \mathscr{O}_{X}\left(-\left(q^{2}-1\right) H+(q-1) E\right) \rightarrow 0
$$

splits (canonically) if and only if $q \neq 2$.

Proof. First let us remark that if the sequence (11) splits then the splitting $\mathscr{O}_{X}\left(-\left(q^{2}-1\right) H+(q-1) E\right) \rightarrow$ $T_{X}(-\log D)$ is unique (up to multiplication by a non-zero constant). To prove this one needs to remark that $\operatorname{Hom}\left(\mathscr{O}_{X}\left(-\left(q^{2}-1\right) H+(q-1) E\right), \mathscr{O}_{X}(-(q-1) H)\right)=H^{0}\left(\mathscr{O}_{X}\left(\left(q^{2}-q\right) H-(q-1) E\right)\right)=0$. The proof is the same as that of [13, Lemma 2.6].

Note that after push-forward by $\pi$ the above sequence becomes the sequence

$$
0 \rightarrow \mathscr{O}_{X}(-(q-1)) \rightarrow T_{\mathbb{P}^{2}}(-\log B) \rightarrow \mathscr{O}_{\mathbb{P}^{2}}\left(-\left(q^{2}-1\right)\right) \rightarrow 0,
$$

which always splits by Proposition 9.1 Now the splitting $\mathscr{O}_{\mathbb{P}^{2}}\left(-\left(q^{2}-1\right)\right) \rightarrow T_{\mathbb{P}^{2}}(-\log B)$ of this sequence can be written (up to multiplication by a constant) as $\eta=\theta_{2}+F \theta_{1}$ for some homogeneous polynomial $F \in k\left[x_{0}, x_{1}, x_{2}\right]$ of degree $q^{2}-q$. The sequence (1) splits if and only if for some $F$ the (rational) vector field $\pi^{*} \eta$ defines splitting. As before let us consider subset $U=D_{+}\left(x_{0}\right) \subset \mathbb{P}^{2}$ with affine coordinates $t_{1}=x_{1} / x_{0}$ and $t_{2}=x_{2} / x_{0}$. Let us take an $\mathbb{F}_{q}$-point $P=\left(a_{1}, a_{2}\right) \in U$. Let $u_{1}, u_{2}$ be the affine coordinates on $U$ given by $u_{i}=t_{i}-a_{i}$ for $i=1,2$. Then we can write

$$
\left.\eta\right|_{U}=\sum_{i=1}^{2}\left(\left(u_{i}^{q^{2}}-1\right)-F\left(1, a_{1}+u_{1}, a_{2}+u_{2}\right)\left(u_{i}^{q-1}-1\right)\right) u_{i} \frac{\partial}{\partial u_{i}} .
$$

Let $V \subset U$ be an open subset containing only one $\mathbb{F}_{q}$-rational point $P$ and let $\tilde{V}=\pi^{-1}(V)$. On an affine chart of $\tilde{V}$ with coordinates $s_{1}, s_{2}$ such that $\pi^{*} u_{1}=s_{1}$ and $\pi^{*} u_{2}=s_{1} s_{2}$ we can write

$$
\begin{aligned}
&\left.\pi^{*} \eta\right|_{\tilde{V}}=\left(\left(s_{1}^{q^{2}}-1\right)-F\left(1, a_{1}+s_{1}, a_{2}+s_{1} s_{2}\right)\left(s_{1}^{q-1}-1\right)\right) s_{1} \frac{\partial}{\partial s_{1}} \\
&+s_{1}^{q-1}\left(s_{1}^{q^{2}-q}\left(s_{2}^{q^{2}-1}-1\right)-F\left(1, a_{1}+s_{1}, a_{2}+s_{1} s_{2}\right)\left(s_{2}^{q-1}-1\right)\right) s_{2} \frac{\partial}{\partial s_{2}}
\end{aligned}
$$

$\pi^{*} \eta$ defines the map $\mathscr{O}_{X}\left(-\left(q^{2}-1\right) H\right) \rightarrow T_{X}(-\log D)$ which defines splitting of the sequence (1) on $\tilde{V}$ if and only if $\left(\left(s_{1}^{q^{2}}-1\right)-F\left(1, a_{1}+s_{1}, a_{2}+s_{1} s_{2}\right)\left(s_{1}^{q-1}-1\right)\right)$ vanishes up to order $(q-1)$ along the exceptional divisor given by $s_{1}=0$ (for all $P \in \mathbb{P}^{2}\left(\mathbb{F}_{q}\right)$ ). This happens (on $\tilde{V}$ ) if and only if $F\left(1, a_{1}, a_{2}\right)=1$. It follows that the sequence (1) splits if and only if there exists a homogeneous polynomial $F \in k\left[x_{0}, x_{1}, x_{2}\right]$ of degree $q^{2}-q$ such that

$$
F\left(a_{0}, a_{1}, a_{2}\right)=1
$$


for all $\left(a_{0}, a_{1}, a_{2}\right) \in \mathbb{F}_{q}^{3}-\{0\}$ (note that if $a_{0} \neq 0$ then $F\left(a_{0}, a_{1}, a_{2}\right)=a_{0}^{q^{2}-q} F\left(1, a_{1} / a_{0}, a_{2} / a_{0}\right)=1$; similarly we have equalities for other charts). If $q=2$ then such a polynomial does not exists as every conic defined over $\mathbb{F}_{q}$ has an $\mathbb{F}_{q}$-rational point (this follows from Chevalley-Warning theorem). Now we claim that if $q \neq 2$ then there exists a degree $q$ plane projective curve defined over $\mathbb{F}_{q}$ with no $\mathbb{F}_{q}$-rational points. This is equivalent to finding a homogeneous polynomial $G \in k\left[x_{0}, x_{1}, x_{2}\right]$ of degree $q$ that has no zeroes in $k^{3}-\{0\}$. To find it let us choose an extension $k \subset k^{\prime}$ of degree $q$. Then the norm map $N_{k^{\prime} / k}: k^{\prime} \rightarrow k$ is given by a homogenous polynomial of degree $q$ in $q$ variables. Restricting it to a 3-dimensional $k$-vector subspace of $k^{\prime}$ and choosing some coordinates we get the required polynomial $G$. Now the required homogeneous polynomial can be obtained by setting $F=G^{q-1}$.

Remark 11.3. Note that if $q>2$ then there exists only one polynomial $F \in k\left[x_{0}, x_{1}, x_{2}\right]$ of degree $q^{2}-q$ such that

$$
F\left(a_{0}, a_{1}, a_{2}\right)=1
$$

for all $\left(a_{0}, a_{1}, a_{2}\right) \in \mathbb{F}_{q}^{3}-\{0\}$. This is non-obvious from the construction of this polynomial and it follows from uniqueness (up to multiplication by a constant) of splitting of the sequence (1).

Note that $\tilde{B}$ is a sum of $\left(q^{2}+q+1\right)$ curves $\tilde{L}_{i} \simeq \mathbb{P}^{1}$ with self-intersection $\tilde{L}_{i}^{2}=-q$. Let $f: X \rightarrow Y$ be the contraction of $\tilde{L}_{1}, \ldots, \tilde{L}_{q^{2}+q+1}$ to a set of points $Q_{1}, \ldots, Q_{q^{2}+q+1}$. Let us also set $E_{Y}=f(E)$. Since $E_{Y}$ is an effective $\mathbb{Q}$-Cartier Weil divisor and $\rho(Y)=1$, it is also ample. Clearly, $Y$ has $\mathbb{Q}$-factorial klt singularities at $Q_{i}$ of the type considered in Section 13 . We show that $\hat{\Omega}_{Y}$ contains an ample line bundle. Since $\Omega_{X}$ does not contain big line bundles, we see that $f_{*} \Omega_{X} \neq \hat{\Omega}_{Y}$ as predicted by Proposition 13.2

PROPOSITION 11.4. The sheaf $f_{*} \Omega_{X}(\log \tilde{B})$ is reflexive and we have a short exact sequence

$$
0 \rightarrow \mathscr{O}_{Y}\left(B_{1}\right) \rightarrow \hat{\Omega}_{Y} \rightarrow \mathscr{O}_{Y}\left(B_{2}\right) \rightarrow 0
$$

where $B_{1}$ is an ample Cartier divisor and $B_{2}$ is an anti-ample $\mathbb{Q}$-Cartier Weil divisor.

Proof. Since we have

$$
\left(\left(q^{2}-1\right) H-(q-1) E\right) \tilde{L}_{i}=0,
$$

locally around $\tilde{L}_{i}$, the sheaf $\mathscr{O}_{X}\left(\left(q^{2}-1\right) H-(q-1) E\right)$ is isomorphic to $\mathscr{O}_{X}$. In particular, $f_{*} \mathscr{O}_{X}\left(\left(q^{2}-\right.\right.$ $1) H-(q-1) E)$ is locally free and $R^{1} f_{*} \mathscr{O}_{X}\left(\left(q^{2}-1\right) H-(q-1) E\right)=0$. Since

$$
((q-1) H-E) \tilde{L}_{i}=-2
$$

Lemma 13.1 shows that $f_{*} \mathscr{O}_{X}((q-1) H-E)$ is reflexive. Let us recall that $\left(q^{2}+q+1\right) H \sim \tilde{B}+(q+1) E$. Hence we have

$$
\left(q^{2}-1\right) H-(q-1) E \sim \mathbb{Q} \frac{q^{2}-q}{q^{2}+q+1} E+\frac{q^{2}-1}{q^{2}+q+1} \tilde{B}
$$

and

$$
(q-1) H-E \sim_{\mathbb{Q}}-\frac{q+2}{q^{2}+q+1} E+\frac{q-1}{q^{2}+q+1} \tilde{B} .
$$

Therefore $B_{1}=f_{*}\left(\left(q^{2}-1\right) H-(q-1) E\right) \sim_{\mathbb{Q}} \frac{q^{2}-q}{q^{2}+q+1} E_{Y}$ is ample and $B_{2}=f_{*}((q-1) H-E) \sim_{\mathbb{Q}}-\frac{q+2}{q^{2}+q+1} E_{Y}$ is anti-ample. Now the required short exact sequence can be obtained by pushing down the second exact sequence from Proposition 11.1

The above proposition completes the proof of Theorem 1.4

Example 11.5. Now we can use the 1-foliations from Section 10 to give a simple interpretation of Theorem 1.2 in the case $n=2$ and $k=\mathbb{F}_{p}$. Let us recall that the map $\pi: \tilde{X}=X \rightarrow \mathbb{P}^{2}$ is the blow up along $\mathbb{P}^{2}\left(\mathbb{F}_{p}\right)$ and the map $f$ is the contraction of all the strict transforms of the $\mathbb{F}_{p}$-lines on $\mathbb{P}^{2}$. The remaining maps can be identified as follows. The map $\varphi: \mathbb{P}^{2} \rightarrow Y$ is the quotient by 1 -foliation defined by $\mathscr{F}_{1} \subset T_{\mathbb{P} 2}$, the map $\varphi_{X}: X \rightarrow X$ is the quotient by the 1 -foliation $\tilde{\mathscr{F}}_{1} \subset T_{X}$ and the map $\varphi_{Y}: Y \rightarrow \mathbb{P}^{2}$ is the quotient by the 1-foliation $\mathscr{O}_{Y}\left(-B_{2}\right) \subset T_{Y}$ coming by dualization from Proposition 11.4. The necessary computations are left to the reader or they can be found in the preprint version of the paper. 
Note that if $q=p$ then $(p+1) H-E$ and $H$ are both globally generated and they define maps contracting different curves. Therefore $a((p+1) H-E)+b H$ is ample for any positive real numbers $a$ and $b$. In particular $A=(p+\sqrt{p}+1) H-E$ is ample. One can easily check that $\varphi_{X}^{*} A=\sqrt{p} A$, so $\varphi_{X}: X \rightarrow X$ is a polarized endomorphism. However, this polarization is an $\mathbb{R}$-divisor and it cannot be chosen to be a $\mathbb{Q}$-divisor.

In fact, we have the following more general lemma suggested by De-Qi Zhang:

LEMMA 11.6. Let $\varphi: X \rightarrow X$ be an endomorphism of a scheme $X$ and let us assume that for some $n \geq 1$, $\lambda \in \mathbb{R}$ and some line bundle $L$ we have $\left(\varphi^{n}\right)^{*} L \equiv \lambda^{n} L$. If we set $M=\left(L+\frac{1}{\lambda} \varphi^{*} L+\ldots+\frac{1}{\lambda^{n-1}}\left(\varphi^{n-1}\right)^{*} L\right)$ then we have $\varphi^{*} M \equiv \lambda M$. In particular, if $\varphi^{n}$ is polarized then $\varphi$ is also polarized.

The proof is an easy computation left to the reader.

Let $\varphi: X \rightarrow X$ be an endomorphism and let $H$ be an ample divisor such that $\varphi^{*} H \equiv \lambda H$. Let us set $X^{n}:=\underbrace{X \times \ldots \times X}_{n}$. Then we can consider the map $\tilde{\varphi}: X^{n} \rightarrow X^{n}$ given by

$$
\tilde{\varphi}\left(x_{1}, \ldots, x_{n}\right)=\left(\varphi\left(x_{n}\right), x_{1}, \ldots, x_{n-1}\right) .
$$

Let us set $A=(H, \ldots, H)$. Then we have $\tilde{\varphi}^{n}=\underbrace{\varphi \times \ldots \times \varphi}_{n}$ and $\left(\tilde{\varphi}^{n}\right)^{*} A \equiv \lambda A$. Therefore $\left(\tilde{\varphi}^{n}\right)^{*} A \equiv \lambda A$ and Lemma 11.6 implies that there exists an ample $\mathbb{R}$-divisor $\tilde{A}$ such that $\tilde{\varphi}^{*} \tilde{A} \equiv \sqrt[n]{\lambda} \cdot \tilde{A}$. Clearly, the same equality holds if we restrict $\tilde{A}$ to any subvariety $Y \subset X^{n}$ preserved by $\tilde{\varphi}$. However, it is usually difficult to find such a subvariety.

The morphism $\varphi_{X}: X \rightarrow X$ from Theorem 1.2 is obtained by this construction applied to the Frobenius morphism $\operatorname{Fr}_{\mathbb{P}^{n}}: \mathbb{P}^{n} \rightarrow \mathbb{P}^{n}$.

\section{A new non-liftable Calabi-Yau threefold in characteristic 2}

Let us consider the 3 -dimensional case, i.e. $n=3$, and let $\tilde{f}: \tilde{X} \rightarrow \tilde{Y}=\tilde{X} / \tilde{\mathscr{F}}_{2}$ be the quotient by 1 -foliation. The main aim of this section is to prove that for $p=q=2$ the obtained variety $\tilde{Y}$ is a new example of a smooth projective Calabi-Yau 3-fold that does not lift to characteristic 0 (see Theorem 12.4). To study this quotient we need some preparatory lemmas.

LEMMA 12.1. We have a short exact sequence

$$
0 \rightarrow \mathscr{O}_{\tilde{X}}\left(-(q-1) H+D_{V}^{3}\right) \rightarrow \tilde{\mathscr{F}}_{2} \rightarrow \mathscr{O}_{\tilde{X}}\left(-\left(q^{2}-1\right) H+(q-1) D_{V}^{3}+D_{V}^{2}\right) \rightarrow 0
$$

Proof. By Proposition 10.1 and Proposition 10.2 we know that $T_{\tilde{X}} / \tilde{\mathscr{F}}_{i}$ are locally free for $i=1,2$. Therefore the sequence

$$
0 \rightarrow \tilde{\mathscr{F}}_{2} / \tilde{\mathscr{F}}_{1} \rightarrow T_{\tilde{X}} / \tilde{\mathscr{F}}_{1} \rightarrow T_{\tilde{X}} / \tilde{\mathscr{F}}_{2} \rightarrow 0
$$

shows that $\tilde{\mathscr{F}}_{2} / \tilde{\mathscr{F}}_{1}$ is a line bundle. Let us recall that the same propositions imply that $\tilde{\mathscr{F}}_{1} \simeq \mathscr{O}_{\tilde{X}}(-(q-$ 1) $\left.H+D_{V}^{3}\right)$ and

$$
\operatorname{det} \tilde{\mathscr{F}}_{2} \simeq \mathscr{O}_{\tilde{X}}\left(-(q-1)(q+2) H+q D_{V}^{3}+D_{V}^{2}\right) .
$$

Hence the required sequence follows from

$$
\tilde{\mathscr{F}}_{2} / \tilde{\mathscr{F}}_{1} \simeq \operatorname{det}\left(\tilde{\mathscr{F}}_{2} / \tilde{\mathscr{F}}_{1}\right) \simeq\left(\operatorname{det} \tilde{\mathscr{F}}_{2}\right) \otimes \tilde{\mathscr{F}}_{1}^{-1} \simeq \mathscr{O}_{\tilde{X}}\left(-\left(q^{2}-1\right) H+(q-1) D_{V}^{3}+D_{V}^{2}\right) .
$$

LEMMA 12.2. $\tilde{Y}$ is a smooth projective 3 -fold with $H^{1}\left(\mathscr{O}_{\tilde{Y}}\right)=0$. 
Proof. Smoothness of $\tilde{Y}$ is a consequence of the fact that $\tilde{\mathscr{F}}_{2}$ is a smooth 1-foliation.

By Lemma 12.1 we have a short exact sequence

$$
0 \rightarrow \mathscr{O}_{\tilde{X}}\left(\left(q^{2}-1\right) H-(q-1) D_{V}^{3}-D_{V}^{2}\right) \rightarrow \tilde{\mathscr{F}}_{2}^{*} \rightarrow \mathscr{O}_{\tilde{X}}\left((q-1) H-D_{V}^{3}\right) \rightarrow 0 .
$$

But $\left.\pi_{*} \mathscr{O}_{\tilde{X}}\left(\left(q^{2}-1\right) H-(q-1) D_{V}^{3}-D_{V}^{2}\right)\right) \subset I_{Z_{2}}\left(q^{2}-1\right)$ and by Corollary $3.2 H^{0}\left(I_{Z_{2}}\left(q^{2}-1\right)\right)=0$, so $H^{0}\left(\mathscr{O}_{\tilde{X}}\left(\left(q^{2}-1\right) H-(q-1) D_{V}^{3}-D_{V}^{2}\right)\right)=0$. Similarly, we have $\pi_{*} \mathscr{O}_{\tilde{X}}\left((q-1) H-D_{V}^{3}\right) \subset I_{Z_{3}}(q-1)$ and again by Corollary 3.2 we have $H^{0}\left(I_{Z_{3}}(q-1)\right)=0$, so $H^{0}\left(\mathscr{O}_{\tilde{X}}\left((q-1) H-D_{V}^{3}\right)\right)=0$. It follows that $H^{0}\left(\tilde{\mathscr{F}}_{2}^{*}\right)=0$. Now by Lemma 2.6 we have an exact sequence

$$
0 \rightarrow \mathscr{O}_{\tilde{Y}} \rightarrow \tilde{f}_{*} \mathscr{O}_{\tilde{X}} \rightarrow \tilde{f}_{*} \tilde{\mathscr{F}}_{2}^{*}
$$

This shows that $H^{0}\left(\tilde{f}_{*} \mathscr{O}_{\tilde{X}} / \mathscr{O}_{\tilde{Y}}\right) \subset H^{0}\left(\tilde{f}_{*} \tilde{\mathscr{F}}_{2}^{*}\right)=H^{0}\left(\tilde{\mathscr{F}}_{2}^{*}\right)=0$. But we have an exact sequence

$$
H^{0}\left(\tilde{f}_{*} \mathscr{O}_{\tilde{X}} / \mathscr{O}_{\tilde{Y}}\right) \rightarrow H^{1}\left(\mathscr{O}_{\tilde{Y}}\right) \rightarrow H^{1}\left(\tilde{f}_{*} \mathscr{O}_{\tilde{X}}\right)=0
$$

so $H^{1}\left(\mathscr{O}_{\tilde{Y}}\right)=0$.

There exists a radicial degree $p$ map $\tilde{g}: \tilde{Y} \rightarrow \tilde{X}^{(1)}$ such that $\tilde{g} \circ \tilde{f}=F_{\tilde{X} / k}$. We have $T_{\tilde{X} / \tilde{Y}}=\tilde{\mathscr{F}}$ and the sheaf $\mathscr{L}:=T_{\tilde{Y} / \tilde{X}^{(1)}}$ is a line bundle on $\tilde{Y}$. We also have two short exact sequences

$$
0 \rightarrow \tilde{\mathscr{F}}_{2} \rightarrow T_{\tilde{X}} \rightarrow \tilde{f}^{*} \mathscr{L} \rightarrow 0
$$

and

$$
0 \rightarrow \mathscr{L} \rightarrow T_{\tilde{Y}} \rightarrow(\sigma \circ \tilde{g})^{*} \tilde{\mathscr{F}}_{2} \rightarrow 0,
$$

where $\sigma: \tilde{X}^{(1)} \rightarrow \tilde{X}$ is the canonical map coming from base change by the Frobenius over the base field.

LEMMA 12.3. We have $H^{0}\left(T_{\tilde{Y}}\right)=0$.

Proof. By Lemma $12.1 F_{\tilde{X}}^{*} \tilde{\mathscr{F}}_{2}$ has a filtration whose quotients are two line bundles, whose intesection with $H^{2}$ is strictly negative. Therefore $H^{0}\left(F_{\tilde{X}}^{*} \tilde{\mathscr{F}}_{2}\right)=0$. On the other hand, we have a short exact sequence

$$
0 \rightarrow \tilde{\mathscr{L}} \rightarrow \tilde{f}^{*} T_{\tilde{Y}} \rightarrow F_{\tilde{X}}^{*} \tilde{\mathscr{F}} 2 \rightarrow 0
$$

where $\tilde{\mathscr{L}}=\tilde{f}^{*} \mathscr{L}$. So it is sufficient to show that $H^{0}(\tilde{\mathscr{L}})=0$. Then $H^{0}\left(\tilde{f}^{*} T_{\tilde{Y}}\right)=0$, which by the projection formula implies vanishing of $H^{0}\left(T_{\tilde{Y}}\right)$.

By Proposition 10.1 we get

$$
\tilde{\mathscr{L}} \simeq \mathscr{O}_{\tilde{X}}\left(\left(q^{2}+q+2\right) H-(q+2) D_{V}^{3}-2 D_{V}^{2}\right) .
$$

Let $\Pi \subset \mathbb{P}(V)$ be an $\mathbb{F}_{q}$-rational plane and let $\tilde{\Pi} \subset \tilde{X}$ be its strict transform. The map $\tilde{\Pi} \rightarrow \Pi$ is the blow up of $\Pi$ at $\Pi\left(\mathbb{F}_{q}\right)$. Note that the restriction of $D_{V}^{2}$ to $\tilde{\Pi}$ is the strict transform $\tilde{B}$ of all $\mathbb{F}_{q}$-lines contained in $\Pi$ and the restriction of $D_{V}^{3}$ to $\tilde{\Pi}$ is the exceptional divisor $E$ of $\tilde{\Pi} \rightarrow \Pi$. Since $\tilde{B}+\left.(q+1) E \sim\left(q^{2}+q+1\right) H\right|_{\tilde{\Pi}}$ we have

$$
\left.\tilde{\mathscr{L}}\right|_{\tilde{\Pi}} \simeq \mathscr{O}_{\tilde{\Pi}}\left(\left(q^{2}+q+2\right) H-(q+2) D_{V}^{3}-2 D_{V}^{2}\right) \simeq \mathscr{O}_{\tilde{\Pi}}(-q((q+1) H-E)) .
$$

Since $(q+1) H-E$ is nef on $\tilde{\Pi}$, we have $H^{0}\left(\left.\tilde{\mathscr{L}}\right|_{\tilde{\Pi}}\right)=0$. Note that if $\Pi_{1}$ and $\Pi_{2}$ are two $\mathbb{F}_{q}$-rational planes then they intersect along an $\mathbb{F}_{q}$-rational line $L$. By Lemma $2.4 \tilde{X}$ dominates the blow up of $\mathbb{P}(V)$ along $L$, so $\tilde{\Pi}_{1}$ and $\tilde{\Pi}_{2}$ do not intersect. So we have a short exact sequence

$$
\left.0 \rightarrow \tilde{\mathscr{L}}\left(-D_{V}^{1}\right) \rightarrow \tilde{\mathscr{L}} \rightarrow \underset{L \in \mathscr{L}(V), \operatorname{codim} L=1}{\bigoplus} \tilde{\mathscr{L}}\right|_{D_{L}} \rightarrow 0
$$

which by the above implies that

$$
H^{0}(\tilde{\mathscr{L}})=H^{0}\left(\tilde{\mathscr{L}}\left(-D_{V}^{1}\right)\right) .
$$

But $c_{1}\left(\tilde{\mathscr{L}}\left(-D_{V}^{1}\right)\right) H^{2}<0$, so $H^{0}\left(\tilde{\mathscr{L}}\left(-D_{V}^{1}\right)\right)=0$. 
THEOREM 12.4. If $p=q=2$ then the following conditions are satisfied:

1. $\tilde{Y}$ is a smooth, projective Calabi-Yau 3-fold,

2. $\tilde{Y}$ is unirational,

3. $b_{2}(\tilde{Y})=51$ and $h^{1}\left(\mathscr{O}_{\tilde{Y}}\right)=h^{2}\left(\mathscr{O}_{\tilde{Y}}\right)=0$,

4. $h^{0}\left(T_{\tilde{Y}}\right)=0$,

5. $T_{\tilde{Y}}$ is not semistable with respect to some ample polarizations,

6. $\tilde{Y}$ does not admit a formal lifting to characteristic zero.

Proof. Since $p=q=2$ by [7, Corollary 3.4] we have

$$
\tilde{f}^{*} K_{\tilde{Y}}=K_{\tilde{X}}-(p-1) c_{1}\left(\operatorname{det} \tilde{\mathscr{F}}_{2}\right)=0 .
$$

Therefore $p K_{\tilde{Y}}=0$.

By Lemma 12.2 we have $h^{1}\left(\mathscr{O}_{\tilde{Y}}\right)=0$. By the Riemann-Roch theorem we get

$$
\chi\left(\mathscr{O}_{\tilde{Y}}\right)=\frac{1}{24} \int_{\tilde{Y}} c_{1}\left(T_{\tilde{Y}}\right) c_{2}\left(T_{\tilde{Y}}\right)=0 .
$$

Hence we have

$$
h^{0}\left(K_{\tilde{Y}}\right)=h^{3}\left(\mathscr{O}_{\tilde{Y}}\right)=h^{2}\left(\mathscr{O}_{\tilde{Y}}\right)+1 \geq 1,
$$

which implies that $K_{\tilde{Y}}=0$ and $h^{2}\left(\mathscr{O}_{\tilde{Y}}\right)=0$.

Let $X_{0}$ be the blow up of $\mathbb{P}^{3}$ along $\mathbb{P}^{3}\left(\mathbb{F}_{2}\right)$, i.e., in 15 points. Then $\tilde{X}$ is the blow up of $X_{0}$ along the strict transforms of all $\mathbb{F}_{2}$-lines in $\mathbb{P}^{3}$, i.e., along 35 disjoint smooth rational curves. Therefore $b_{2}(\tilde{X})=$ $1+15+35=51$. Since $\tilde{f}: \tilde{X} \rightarrow \tilde{Y}$ is radicial, $\tilde{Y}$ is unirational and we have $b_{2}(\tilde{Y})=b_{2}(\tilde{X})=51$.

By Lemma 12.3 we have $h^{0}\left(T_{\tilde{Y}}\right)=0$.

Let us set $g=\sigma \circ \tilde{g}$. Since $\tilde{f} \circ g=F_{\tilde{Y}}$ we have

$$
p \mu_{g^{*} H}(\mathscr{L})=\mu_{g^{*} H}\left(F_{\tilde{Y}}^{*} \mathscr{L}\right)=\operatorname{deg} \tilde{g} \cdot \mu_{H}\left(\tilde{f}^{*} \mathscr{L}\right)=p \cdot c_{1}\left(\tilde{f}^{*} \mathscr{L}\right) H^{2}=p\left(q^{2}+q+2\right)>0 .
$$

In particular, $\mu_{g^{*} H}(\mathscr{L})>\mu_{g^{*} H}\left(T_{\tilde{Y}}\right)=0$ and $T_{\tilde{Y}}$ is $g^{*} H$-unstable. Then $T_{\tilde{Y}}$ is $A$-unstable for ample polarizations $A$ that are close to the divisor $\tilde{g}^{*} H$ (which is only nef and big) in the (rational) Neron-Severi group of $\tilde{Y}$.

The fact that $\tilde{Y}$ does not admit a formal lifting to characteristic zero follows by the same arguments as that of [30, Theorem 2.1].

Remark 12.5. One can expect that similarly to Hirokado's example, the above example is arithmetically rigid (see [7, Theorem A]). Analogously to [ibid.], one should compute all the Hodge numbers of $\tilde{Y}$ and prove that one can lift a group of automorphisms, but it seems that there is no short and easy way to do so.

\section{Cones over projective spaces}

Let us recall the following lemma which comes from [33, Lemma 4.14] (the proof works also in positive characteristic and in higher dimensions).

LEMMA 13.1. Let $Z$ be an affine variety with singularity at $z$ and let $f: \tilde{Z} \rightarrow Z$ be resolution of singularities. Let us assume that $f$ is an isomorphism on $Z-\{z\}$. Let $M$ be a line bundle on $\tilde{Z}$ such that $L^{\otimes m} \simeq \mathscr{O}_{\tilde{Z}}(D)$ for some positive integer $m$ and an effective exceptional divisor $D$. Then $f_{*} L$ is reflexive.

Let $g: \tilde{Z}=\mathbb{V}\left(\mathscr{O}_{\mathbb{P}^{m}}(-d)\right)=$ Spec $\bigoplus_{i>0} \mathscr{O}_{\mathbb{P}^{m}}(d)^{\otimes i} \rightarrow \mathbb{P}^{m}$ be the total space of $\mathscr{O}_{\mathbb{P}^{m}}(d)$ and let $S$ be its zero section. Let $f: \tilde{Z} \rightarrow Z=\operatorname{Spec} \bigoplus_{i \geq 0} H^{0}\left(\mathbb{P}^{m}, \mathscr{O}_{\mathbb{P}^{m}}(d)^{\otimes i}\right)$ be the contraction of $S$ to a cone over $\left(\mathbb{P}^{m}, \mathscr{O}_{\mathbb{P}^{m}}(d)\right)$. 
PROPOSITION 13.2. Z has only klt singularities and $f_{*} \Omega_{\tilde{Z}}(\log S)$ is reflexive. If the base field has positive characteristic $p$ and $p$ divides $d$ then $f_{*} \Omega_{\tilde{Z}}$ is not reflexive. Moreover, $f_{*} \Omega_{\tilde{Z}}(\log S)$ is locally free if $p=d=2$ and $m=1$.

Proof. A simple computation shows that $K_{\tilde{Z}}+S=f^{*} K_{Z}+\frac{m+1}{d} S$, so $Z$ has only klt singularities. Let us consider an exact sequence

$$
0 \rightarrow \Omega_{\mathbb{P} m}^{1} \rightarrow G \rightarrow \mathscr{O}_{\mathbb{P} m} \rightarrow 0
$$

determined by the class of $\mathscr{O}_{\mathbb{P}^{m}}(d)$ in $H^{1}\left(\Omega_{\mathbb{P}^{m}}^{1}\right)$ via $H^{1}\left(\mathscr{O}_{\mathbb{P}^{m}}^{*}\right) \rightarrow H^{1}\left(\Omega_{\mathbb{P}^{m}}^{1}\right)$. By [32, Proposition 3.3] we have $\Omega_{\tilde{Z}}(\log S) \simeq g^{*} G$. If $p$ divides $d$ then the above sequence splits and $\Omega_{\tilde{Z}}(\log S) \simeq g^{*} \Omega_{\mathbb{P}^{m}} \oplus \mathscr{O}_{\tilde{Z}}$. If $p$ does not divide $d$ then the above sequence does not split and $\Omega_{\tilde{Z}}(\log S) \simeq g^{*} \mathscr{O}_{\mathbb{P} m}(-1)^{\oplus(m+1)}$.

Since $g^{*} \mathscr{O}_{\mathbb{P} m}(-d) \simeq \mathscr{O}_{\tilde{Z}}(S)$, Lemma 13.1 shows that $M=f_{*}\left(g^{*} \mathscr{O}_{\mathbb{P} m}(-1)\right)$ is a rank 1 reflexive sheaf (corresponding to the ideal sheaf of a hyperplane passing through the vertex $z$ of the cone $Z$ ). Euler's exact sequence

$$
0 \rightarrow \Omega_{\mathbb{P} m}^{1} \rightarrow \mathscr{O}_{\mathbb{P} m}(-1)^{\oplus(m+1)} \rightarrow \mathscr{O}_{\mathbb{P} m} \rightarrow 0
$$

leads to the exact sequence

$$
0 \rightarrow f_{*}\left(g^{*} \Omega_{\mathbb{P} m}^{1}\right) \rightarrow M^{\oplus(m+1)} \rightarrow \mathscr{O}_{Z}
$$

which shows that $f_{*}\left(g^{*} \Omega_{\mathbb{P} m}^{1}\right)$ is reflexive. Therefore $f_{*} \Omega_{\tilde{Z}}(\log S)$ is always reflexive. More precisely, $f_{*} \Omega_{\tilde{Z}}(\log S) \simeq f_{*}\left(g^{*} \Omega_{\mathbb{P} m}^{1}\right) \oplus \mathscr{O}_{Z}$ if $p$ divides $d$ and $f_{*} \Omega_{\tilde{Z}}(\log S) \simeq M^{\oplus(m+1)}$ if $p$ does not divide $d$. In particular, $f_{*} \Omega_{\tilde{Z}}(\log S)$ is locally free if $p=d=2$ and $m=1$.

Now let us note that the map $g^{*} \Omega_{\mathbb{P} m}^{1} \rightarrow \Omega_{\tilde{Z}}(\log S)$ factors through the canonical map $g^{*} \Omega_{\mathbb{P} m}^{1} \rightarrow \Omega_{\tilde{Z}}$. Let us assume that $p$ divides $d$. Then $g^{*} \Omega_{\mathbb{P} m}^{1} \rightarrow \Omega_{\tilde{Z}}(\log S)$ splits and hence the sequence

$$
0 \rightarrow g^{*} \Omega_{\mathbb{P} m}^{1} \rightarrow \Omega_{\tilde{Z}} \rightarrow \Omega_{\tilde{Z} / \mathbb{P}^{m}} \rightarrow 0
$$

also splits. It follows that $\Omega_{\tilde{Z}} \simeq \mathscr{O}_{\tilde{Z}}(-S) \oplus g^{*} \Omega_{\mathbb{P}^{m}}^{1}$, so $f_{*} \Omega_{\tilde{Z}} \simeq m_{z} \oplus f_{*}\left(g^{*} \Omega_{\mathbb{P} m}^{1}\right)$ is not reflexive.

The above proposition immediately implies Proposition 1.5

\section{Purely inseparable flops}

In this section we construct an analogue of the celebrated Atiyah's flop. Let us recall that Atiyah's flop is obtained from a quadric singularity $(x y=z w) \subset \mathbb{A}^{4}$ by blowing up along the planes $x=z=0$ and $x=w=0$. The corresponding rational map $X_{1} \rightarrow X_{2}$ between the blow-ups is resolved by the blow up of the singularity along the point $x=y=z=w=0$. Geometrically, this rational map is obtained by taking $\mathbb{P}^{1} \subset X_{1}$ with normal bundle $\mathscr{O}_{\mathbb{P}^{1}}(-1)^{\oplus 2}$, blowing it up and then contracting the exceptional divisor $\mathbb{P}^{1} \times \mathbb{P}^{1}$ in the other direction to $\mathbb{P}^{1}$ with the same normal bundle $\mathscr{O}_{\mathbb{P} 1}(-1)^{\oplus 2}$. In fact, this flop can be seen also when studying the standard Cremona transformation $\mathbb{P}^{3} \rightarrow \rightarrow \mathbb{P}^{3}$ defined by $\left[x_{0}, x_{1}, x_{2}, x_{3}\right] \rightarrow\left[x_{0}^{-1}, x_{1}^{-1}, x_{2}^{-1}, x_{3}^{-1}\right]$ (see, e.g., [22, p. 6014]). Similarly, our "purely inseparable Cremona transformation" leads to a purely inseparable flop described below.

Let $X_{1}$ be a smooth 3-fold defined over an algebraically closed field of characteristic $p$ and let us fix some $q=p^{e}$. Let $C_{1} \subset X_{1}$ be a smooth rational curve with normal bundle $N_{C_{1} \mid X_{1}} \simeq \mathscr{O}_{\mathbb{P}^{1}}(-q)^{\oplus 2}$ and let $p_{1}: X \rightarrow X_{1}$ be the blow up of $X_{1}$ along $C_{1}$. The exceptional divisor $E_{1} \simeq \mathbb{P}^{1} \times \mathbb{P}^{1}$ has normal bundle $N_{E_{1} \mid X} \simeq \mathscr{O}_{\mathbb{P}^{1} \times \mathbb{P}^{1}}(-1,-q)$. Assume that there exists a purely inseparable morphism $\varphi: X \rightarrow X$ exchanging rulings, so that $E_{2}=\varphi\left(E_{1}\right)$ has normal bundle $N_{E_{2} \mid X} \simeq \mathscr{O}_{\mathbb{P}^{1} \times \mathbb{P}^{1}}(-q,-1)$. Then contracting $E_{2}$ along the other ruling we get $p_{2}: X \rightarrow X_{2}$.

Note that since the conormal bundle of $E_{1}$ in $X$ is ample, we can contract $E_{1}$ to a point, i.e., there exists a morphism $f_{1}: X \rightarrow Y$ to a normal algebraic space $Y$ that is an isomorphism outside of $E_{1}$ and contracts $E_{1}$ to a point (see [1, Corollary 6.12]). Clearly, $f_{1}$ factors through $p_{1}$ inducing a contraction $q_{1}: X_{1} \rightarrow Y$. Similarly, we can construct $q_{2}: X_{2} \rightarrow Y$. Then $\varphi: X \rightarrow X$ induces a purely inseparable endomorphism 
$\varphi_{Y}: Y \rightarrow Y$. Summing up, we have the following diagram:

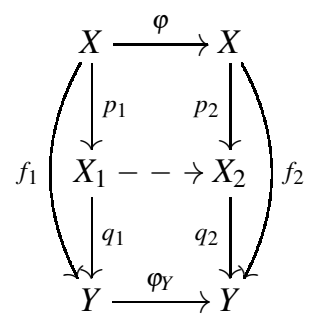

in which the rational map $X_{1} \rightarrow X_{2}$ is a purely inseparable map with indeterminacy locus equal to $C_{1}$. Geometrically, this rational map replaces $C_{1}$ with another smooth rational curve $C_{2}$ with normal bundle $N_{C_{2} \mid X_{2}} \simeq \mathscr{O}_{\mathbb{P}^{1}}(-q)^{\oplus 2}$.

We could also directly try to contract $E_{1} \subset X$ along the other ruling obtaining maps $f_{1}: X \rightarrow Z_{1}$ and $g_{1}: Z_{1} \rightarrow Y$, but then $Z_{1}$ acquires rather bad singularities along the curve $f_{1}\left(E_{1}\right) . Z_{1}$ comes with a purely inseparable morphism $Z_{1} \rightarrow X_{2}$ compatible with $\varphi$ and $\varphi_{Y}$.

In fact, by [1, Corollary 6.13] the above $E_{1} \subset X$ is locally in the étale topology isomorphic to the cylinder over $\left(\mathbb{P}^{1} \times \mathbb{P}^{1}, \mathscr{O}_{\mathbb{P}^{1} \times \mathbb{P}^{1}}(1, q)\right)$. Then locally $Y$ is simply the cone over $\left(\mathbb{P}^{1} \times \mathbb{P}^{1}, \mathscr{O}_{\mathbb{P}^{1} \times \mathbb{P}^{1}}(1, q)\right)$. Then the map $\varphi_{Y}: Y \rightarrow Y$ is induced from the map $\mathbb{P}^{1} \times \mathbb{P}^{1} \rightarrow \mathbb{P}^{1} \times \mathbb{P}^{1}$ sending $(x, y)$ to $\left(y^{q}, x\right)$.

\section{Appendix: Plane curves in positive characteristic}

Let us fix $\bar{k}$-points $\Sigma=\left\{P_{1}, \ldots, P_{m}\right\}$ of $\mathbb{P}^{2}$ and some positive integers $\left(q_{1}, \ldots, q_{m}\right)$. Let $\pi: X \rightarrow \mathbb{P}^{2}$ be the blow up of $\mathbb{P}^{2}$ along $\Sigma$. Let us fix some positive integer $d$ and let us set

$$
B=(d+1) H-\sum q_{i} E_{i},
$$

where $H$ is the pull back of a line on $\mathbb{P}^{2}$ and $E=\sum E_{i}$ is the exceptional divisor of $\pi$. The projection formula implies that

$$
\chi\left(X, K_{X}+B\right)=\chi\left(\mathbb{P}^{2}, m_{P_{1}}^{q_{1}-1} \ldots m_{P_{m}}^{q_{m}-1}(d-2)\right)=\left(\begin{array}{l}
d \\
2
\end{array}\right)-\sum_{i=1}^{m}\left(\begin{array}{c}
q_{i} \\
2
\end{array}\right) .
$$

Moreover, we have

$$
H^{i}\left(X, K_{X}+B\right)=H^{i}\left(\mathbb{P}^{2}, m_{P_{1}}^{q_{1}-1} \ldots m_{P_{m}}^{q_{m}-1}(d-2)\right)
$$

for $i=0,1,2$.

Let $C \subset \mathbb{P}^{2}$ be a reduced plane curve of degree $d$. Let us take as $\Sigma=\left\{P_{1}, \ldots, P_{m}\right\}$ the singular locus of $C$ and let $q_{i}$ be the multiplicity of $P_{i}$ in $C$. Below we recall a certain lemma contained in a part of [21, Exercise 6.8].

Lemma 14.1. There exists an ample $\mathbb{Q}$-divisor $M$ on $X$ such that

$$
H^{1}\left(X, K_{X}+\lceil M\rceil\right)=H^{1}\left(\mathbb{P}^{2}, m_{P_{1}}^{q_{1}-1} \ldots m_{P_{m}}^{q_{m}-1}(d-2)\right) .
$$

Proof. Let $\tilde{C}$ be the strict transform of $C$. Then $\pi^{*} C=\tilde{C}+\sum q_{i} E_{i}$. Let us choose a small $\varepsilon>0$ such that $H-\varepsilon E$ is ample. For some $a$ let us consider the $\mathbb{Q}$-divisor

$$
M=(d+1) H-a \tilde{C}-\sum a\left(q_{i}+\varepsilon\right) E_{i} .
$$

If $0<a<1$ then the numerical equivalence

$$
M \equiv(d+1)(1-a) H+a(H-\varepsilon E)+a\left(d H-\tilde{C}-\sum q_{i} E_{i}\right) \equiv(d+1)(1-a) H+a(H-\varepsilon E)
$$


shows that $M$ is ample. Now let us choose $a<1$ such that $a>\frac{q_{i}}{q_{i}+\varepsilon}$ for every $i$. Then

$$
B=\lceil M\rceil=(d+1) H-\sum q_{i} E_{i},
$$

so

$$
H^{1}\left(X, K_{X}+\lceil M\rceil\right)=H^{1}\left(\mathbb{P}^{2}, m_{P_{1}}^{q_{1}-1} \ldots m_{P_{m}}^{q_{m}-1}(d-2)\right)
$$

as required.

Remark 14.2. Note that even though the support of $\Delta=\lceil M\rceil-M$ need not be a simple normal crossing divisor, the proof of Sakai's lemma [21, Exercise 6.6] shows that if $\tau: X^{\prime} \rightarrow X$ is a $\log$ resolution of $(X, \Delta)$ then vanishing of $H^{1}\left(X^{\prime}, K_{X^{\prime}}+\left\lceil\tau^{*} M\right\rceil\right)$ implies vanishing of $H^{1}\left(X, K_{X}+\lceil M\rceil\right)$. Let $E^{\prime}$ be the exceptional divisor of $\tau$. We can choose a small $\varepsilon^{\prime}>0$ such that $M^{\prime}=\tau^{*} M-\varepsilon^{\prime} E^{\prime}$ is ample and $\left\lceil M^{\prime}\right\rceil=\left\lceil\tau^{*} M\right\rceil$. Therefore if $H^{1}\left(X, K_{X}+\lceil M\rceil\right) \neq 0$ then there exists an ample divisor $M^{\prime}$ on $X^{\prime}$ such that its fractional part $\Delta^{\prime}=\left\lceil M^{\prime}\right\rceil-M^{\prime}$ is a simple normal crossing divisor and $H^{1}\left(X^{\prime}, K_{X^{\prime}}+\left\lceil M^{\prime}\right\rceil\right) \neq 0$.

QUESTIONS 14.3. In the notation as above:

1. Is it true that $\Sigma$ imposes independent conditions on curves of degrees $s \geq d-2$ ?

2. More generally, is it true that

$$
H^{1}\left(\mathbb{P}^{2}, m_{P_{1}}^{q_{1}-1} \ldots m_{P_{m}}^{q_{m}-1}(s)\right)=0
$$

$$
\text { for } s \geq d-2 \text { ? }
$$

3. If $\left(\begin{array}{l}d \\ 2\end{array}\right) \leq \sum_{i=1}^{m}\left(\begin{array}{c}q_{i} \\ 2\end{array}\right)$ (corresponding to $\left.\chi\left(Y, K_{Y}+B\right) \leq 0\right)$ then can the linear system $\left|m_{P_{1}}^{q_{1}} \ldots m_{P_{m}}^{q_{m}}(d)\right|$ contain any reduced curves?

Clearly, in characteristic zero by the Kawamata-Viehweg vanishing the answers to questions 1 and 2 are positive and the answer to question 3 is negative (see [21, Exercise 6.8]). By [3, Theorem 1.1] Kawamata-Viehweg vanishing fails on rational surfaces in positive characteristic. However, one can still hope that answers to the above questions are the same as in characteristic zero.

\section{Acknowledgement}

The author would like to thank. H. Esnault, R. Pink, K. Schwede, V. Srinivas, A. Werner and D. Zhang for useful conversations. In particular, the idea of proof of the first part of Theorem 1.1 is entirely due to $H$. Esnault and V. Srinivas, although the author had not seen their proof of ampleness of $K_{\bar{X}}+D$.

\section{References}

[1] M. Artin, Algebraization of formal moduli. II. Existence of modifications. Ann. of Math. (2) 91 (1970), 88-135.

[2] W. Bruns, U. Vetter, Determinantal rings, Lecture Notes in Mathematics 1327. Springer-Verlag, Berlin, 1988.

[3] P. Cascini, H. Tanaka, Smooth rational surfaces violating Kawamata-Viehweg vanishing, Eur. J. Math. 4 (2018), 162-176.

[4] S. Cynk, D. van Straten, Small resolutions and non-liftable Calabi-Yau threefolds, Manuscripta Math. 130 (2009), 233-249.

[5] C. De Concini, C. Procesi, Wonderful models of subspace arrangements. Selecta Math. (N.S.) 1 (1995), 459-494. 
[6] P. Deligne, G. Lusztig, Representations of reductive groups over finite fields, Ann. of Math. 103 (1976), 103-161.

[7] T. Ekedahl, Foliations and inseparable morphisms. Algebraic geometry, Bowdoin, 1985 (Brunswick, Maine, 1985), 139-149, Proc. Sympos. Pure Math. 46, Part 2, Amer. Math. Soc., Providence, RI, 1987.

[8] T. Ekedahl, On non-liftable Calabi-Yau threefolds, arXiv:0306435v2, preprint, 2004.

[9] D. Goss, Basic structures of function field arithmetic. Ergebnisse der Mathematik und ihrer Grenzgebiete (3), 35. Springer-Verlag, Berlin, 1996. xiv+422 pp.

[10] D. Greb, S. Kebekus, S. Kovács, Th. Peternell, Differential forms on log canonical spaces. Publ. Math. Inst. Hautes Études Sci. 114 (2011), 87-169.

[11] S. H. Hansen, Canonical bundles of Deligne-Lusztig varieties, Manuscripta Math. 98 (1999), 363-375.

[12] R. Hartshorne, Algebraic geometry, Graduate Texts in Mathematics, No. 52. Springer-Verlag, New York-Heidelberg, 1977.

[13] M. Hirokado, A non-liftable Calabi-Yau threefold in characteristic 3, Tohoku Math. J. 51 (1999), 479-487.

[14] G. Hochschild, Simple algebras with purely inseparable splitting fields of exponent 1, Trans. Amer. Math. Soc. 79 (1955), 477-489.

[15] Y. Hu, A compactification of open varieties. Trans. Amer. Math. Soc. 355 (2003), 4737-4753.

[16] D. Huybrechts, M. Lehn, The geometry of moduli spaces of sheaves, Aspects of Mathematics 31, 1997.

[17] S. Iitaka, Algebraic geometry. An introduction to birational geometry of algebraic varieties. Graduate Texts in Mathematics 76. North-Holland Mathematical Library, 24. Springer-Verlag, New York-Berlin, 1982. x+357 pp.

[18] S. Kurul, A. Werner, On endomorphisms of arrangement complements, preprint, arXiv:1708.06260,

[19] J. Kollár, Rational curves on algebraic varieties. Ergebnisse der Mathematik und ihrer Grenzgebiete. 3. Folge. A Series of Modern Surveys in Mathematics, 32. Springer-Verlag, Berlin, 1996. viii +320 pp.

[20] A. Langer, The Bogomolov-Miyaoka-Yau inequality for logarithmic surfaces in positive characteristic, Duke Math. J. 165 (2016), 2737-2769.

[21] R. Lazarsfeld, Lectures on linear series. With the assistance of Guillermo Fernandez del Busto. IAS/Park City Math. Ser., 3, Complex algebraic geometry (Park City, UT, 1993), 161-219, Amer. Math. Soc., Providence, RI, 1997.

[22] J. Lesieutre, Derived-equivalent rational threefolds. Int. Math. Res. Not. IMRN 15 (2015), 60116020 .

[23] L. Li, Wonderful compactification of an arrangement of subvarieties. Michigan Math. J. 58 (2009), 535-563.

[24] D. Mumford, Lectures on curves on an algebraic surface. With a section by G. M. Bergman. Annals of Mathematics Studies, No. 59 Princeton University Press, Princeton, N.J. 1966.

[25] G. A. Mustafin, Nonarchimedean uniformization, Mat. Sb. (N.S.), 1978, Volume 105(147), 207237. 
[26] P. Orlik, H. Terao, Arrangements of hyperplanes. Grundlehren der Mathematischen Wissenschaften 300. Springer-Verlag, Berlin, 1992. xviii+325 pp.

[27] R. Pink, S. Schieder, Compactification of a Drinfeld period domain over a finite field, J. Algebraic Geom. 23 (2014), 201-243.

[28] B. Rémy, A. Thuillier, A. Werner, Automorphisms of Drinfeld half-spaces over a finite field. Compos. Math. 149 (2013), 1211-1224.

[29] A. N. Rudakov, I. R. Shafarevich, Inseparable morphisms of algebraic surfaces. (Russian) Izv. Akad. Nauk SSSR Ser. Mat. 40 (1976), 1269-1307.

[30] S. Schröer, Some Calabi-Yau threefolds with obstructed deformations over the Witt vectors. Compos. Math. 140 (2004), 1579-1592.

[31] A. Seidenberg, Reduction of singularities of the differential equation $A d y=B d x$. Amer. J. Math. 90 (1968), 248-269.

[32] J. Wahl, Equisingular deformations of normal surface singularities. I, Ann. of Math. 104 (1976), 325-356.

[33] J. Wahl, Second Chern class and Riemann-Roch for vector bundles on resolutions of surface singularities, Math. Ann. 295 (1993), 81-110. 